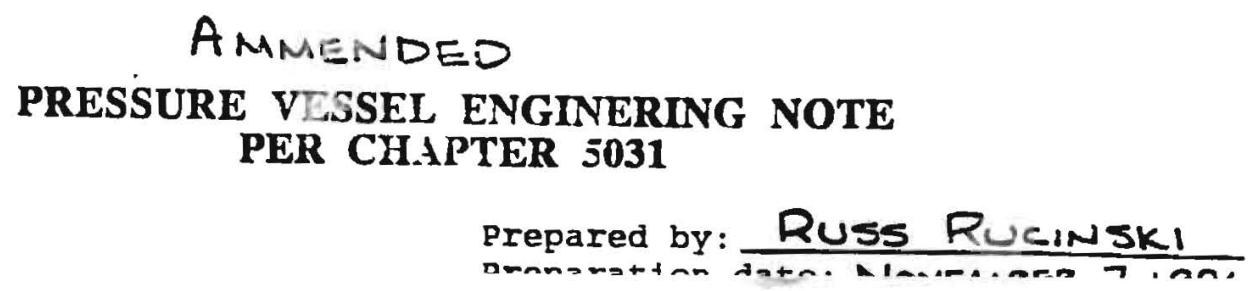

LN2 STORAGE DEWAR \# 39

PRESSURE VESSEL \& VACUUM VESSEL

ENGINEERING NOTE

D-ZERO ENGINEERING NOTE \# 3823.115 EN-464

Author: Russ Rucinski, RD/D-Zero Mech.

January 28, 1997

Approved: Kurt Krempetz

PPD/ETT/D-Zero Mechanical Group leader 
Lab property Numbers): NAL 51464

Lab Location code: $R P I S-325$ (DAB) (obtain from safety officer)

purpose of vessel (s): ProvidE LN Z FOR D-ZERO'S HELIUM REFRIGERATOR

SUPERCONDUCTING SOLENOID, VLPC CRYOSTATS, ANOIOR LAT CALORIMETERS Vessel Capacity/Size: 9000 GAL. Diameter: $120^{\prime \prime}$ Length: $387 "$

Normal operating pressure (OP)_ 35 PSI

MAWP-OP $=43$ PSI

5031TA-6

Fermilab ES\&H Manual

Rev. $3 / 92$ 
List the numbers of all pertinent drawings and the location of the

origirais.

Drawing 4

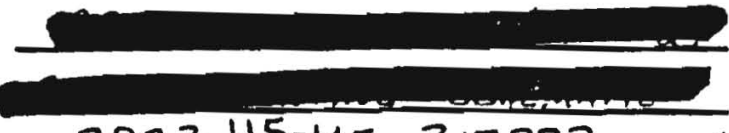

$3823.115-M E-317223$ SHT. 1

(FLOW SCHEMATIC)
Iocation of Oxdodinal

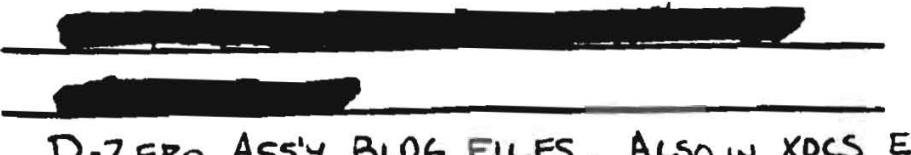

D.ZERD ASS'Y BLDG FILES. ALSO IN XDCS ELECTRONK DRAWING SYSTEM.

\section{Desion Verification}

Does the vessel (s) have a 0 stamp? Yes $X$ No__. If Hes", 1111 out data below and skip page $3 ;$ if "No", fIII out page 3 and skip this page.

Staple photo of 0 stamp plate below.

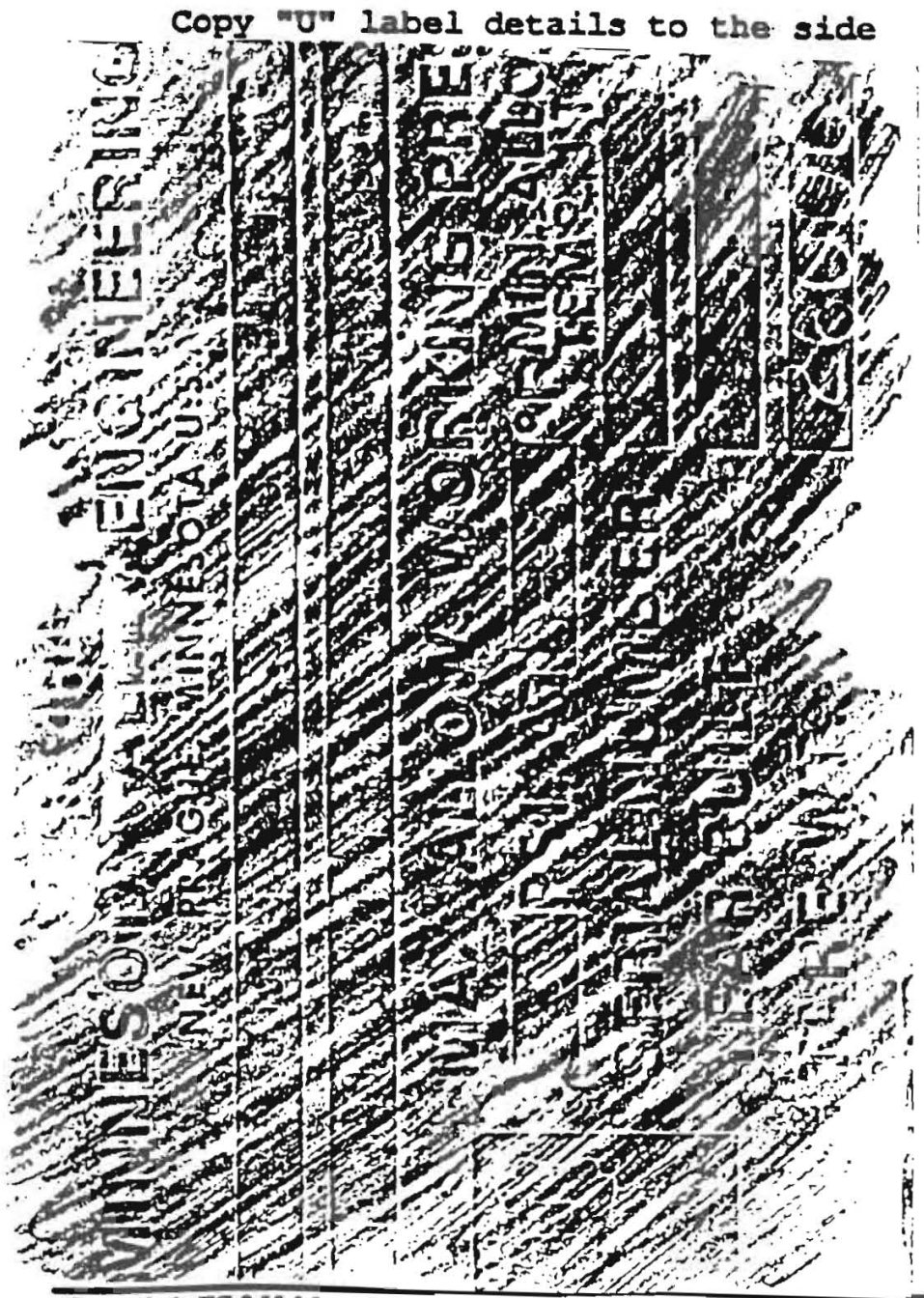

Copy data here: NATIONAL BOARD No. 8602 VLS 9000 INNER VESSEL Only Duplicate Max. WeIGHT of Contents

$104963 \mathrm{lb}$.
MAWP 78 PSLG AT $-32 C^{\circ} \mathrm{F}$
MIN. ALLOW TEMP.
SERIAL NO. 106
YEAR BULT 1985
TARE WT. $26000 \mathrm{lb}$.

Fermilab ES\&H Manual 
3. System Venting Verification Provide the system schematic in the Appendix.

Is it possible to isolate the relief valves by a valve from the vessel?

Yes__ No_ $X$

If "Yes", the system must conform to code rules. Provide an explanation on the appended schematic. (An isolatable vessel, not conforming to code rule is non-compliant under this chapter. )

Is the relief cracking pressure set at or below the M.A.W.P.?

Yes $x$ No_Actual setting 60 PSI

(A "No" response violates this chapter.)

Is the pressure drop of the relief system at maximum anticipated flow such that vessel pressure never rises above the following? (UG 125) Yes $X$ No

1108 of MAWP (one relief) 1168 of MAWP (multiple reliefs) 1218 of MAWP (unexpected heat source)

Provide test or calculational proof in the Appendix. (Non-conforming pressure rises is non-compliant under this chapter.)

List of reliefs and settings:

Manufacturer

CPV DISC

FIKE BURST DISC

ANDERSON GREENWOOD 81B88-8
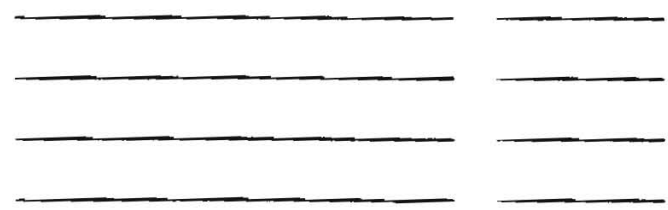

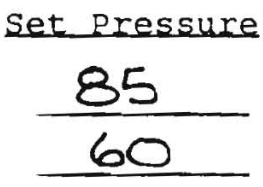

Elow Rate

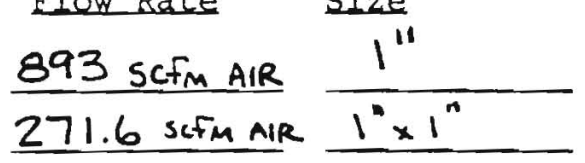

Does the primary relief device follow UG-129? Yes_X No (A "No" response is non-compliant under this chapter)

4. eperating erocedure

Is an operating procedure necessary for the safe operation of this vessel?

Yes___ No___ (If "Yes", it must be appended)

5. Welding Information

Has the vessel been fabricated in a non-code shop? Yes___ No X If "Yes", append a copy of the welding shop statement of welder qualification (Procedure Qualification Record, PQR) which references the Welding procedure Specification (WPS) used to weld this vessel. 
6.

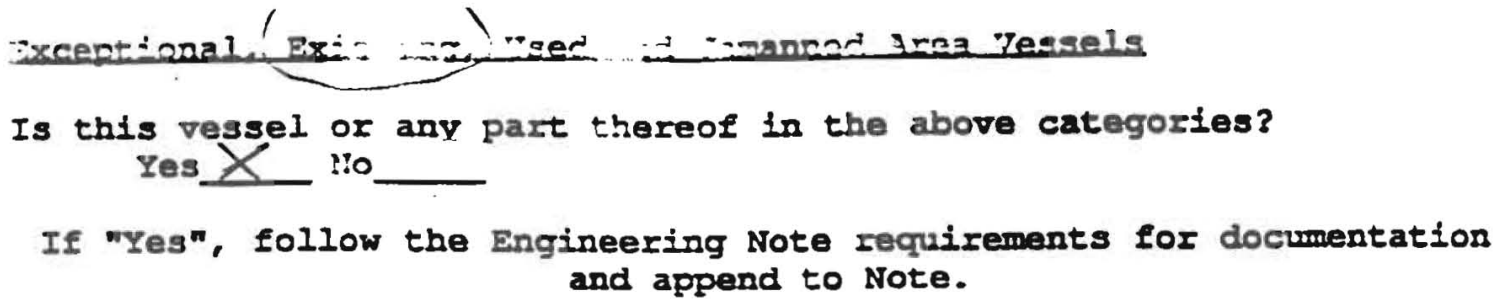

NOTE: THIS IS AN EXISTING DEWAR THAT WAS MOVED FROM THE E- TOG EXPERIMENT (NOW DE-COMMISSIONED) TO D-ZERO. RATHER THAN TRY TO REVISE THE ORIGINAL NOTE, A NEW ENGINEERING NOTE (THIS NOTE) WAS GENERATED. $\left[\begin{array}{l}\text { A COPY OF THE ORIGINAL NOTE CAN } \\ \text { BE OBTAINED FROM THE LABORATORY } \\ \text { PRESSURE VESSEL MASTER FILE MAINTAINED } \\ \text { BY THE ES \&H SECTION. }\end{array}\right]$.

5031TA-10

Fermilab ES\&H Manual

Rev. 3/92 


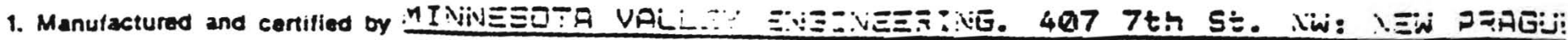

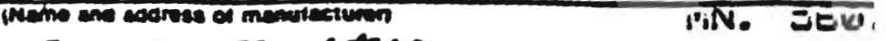

\section{Manufactured for \\ Fermi National Accelerator Labs, Batavia, II 6030}

3. Location of Installation Fermi National Labs, Batavia, IL 60510

VLS 3000 IOA 106 IA

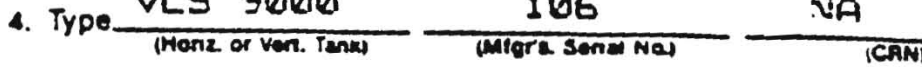

145ะ9อ

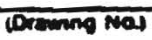

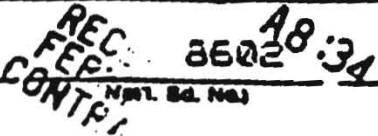

5. The chemical and physical properties of all parts meet the requirements of material specifications of the ASME BOILER AND PRES. SURE VESSEL COOE The design, construction, and workmanship conform to ASME Rules, Section VIII DIyision i i 90 10 $3-04$

$$
10 \text { Adonos (ONO) }
$$

6. Sheil: $5 A 240-304$ Nìt

LUW IMP. SVE. UW E. UnKE!

\section{Sheil:} cosen Can

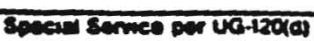

7. Seams: WLD. DEL. EUTT FUIL singin La But!

A.T. (S00 or Fuili)

(a) Mati. SA24Q-3Q44

8. Heads: (a) Matl.

(Soec Na. Great)
.288
a
$8^{2}-5$
$181-9$

\begin{tabular}{|c|c|c|c|c|c|}
\hline & 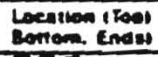 & 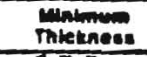 & $\begin{array}{l}\text { Comerenen } \\
\text { Allewence }\end{array}$ & $\begin{array}{c}\text { Cresen } \\
\text { nestua }\end{array}$ & 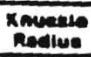 \\
\hline (a) & Tि & 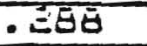 & $\bar{v}$ & ivA & NAG \\
\hline (b) & हाग & - इ8в & र & Nive & NA \\
\hline
\end{tabular}

If removable. bolts used (describe other fastenings)

\section{MAWP 78}

Min. temp (when less than $-20^{\circ} \mathrm{F}$ - $\bar{E}$

10. Nozztes, inspection and safety valve openings:

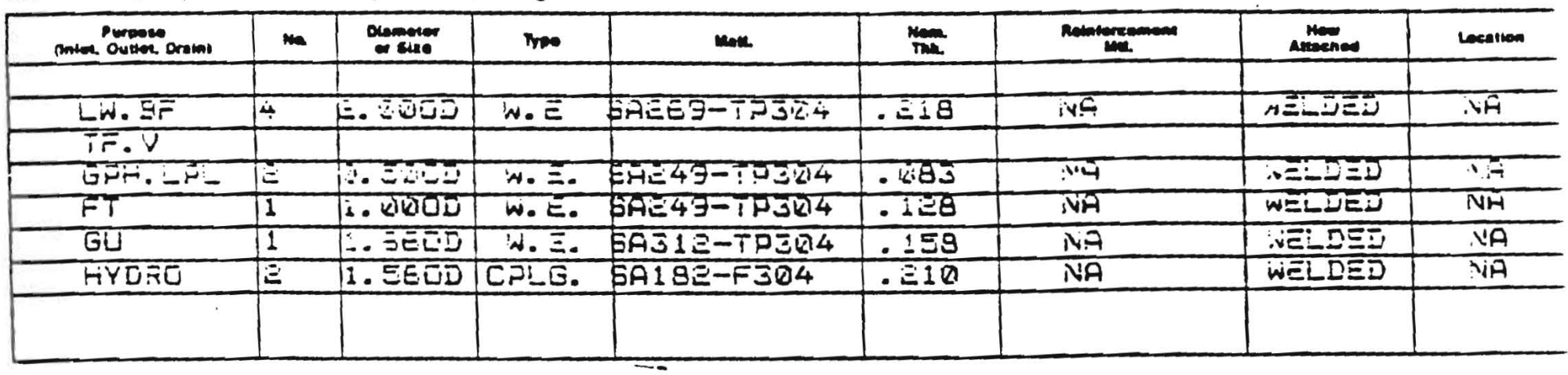

psi at max. temp L IQ _ L 'F. Hydro. pneu., or comb. test pressure

\begin{tabular}{|c|c|c|c|c|}
\hline Onpotien & Cemien & momenom & Dianen & 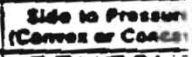 \\
\hline$\Xi: \vdots$ & जिम & NA & iVA & EIUVCAVE \\
\hline $2: 1$ & $\mathrm{NA}$ & NA & NA & CENCAV \\
\hline
\end{tabular}

NDIVE 1Mel. Spec Na. Ge Sis Nas
Leagen (overell) (11. Lint)

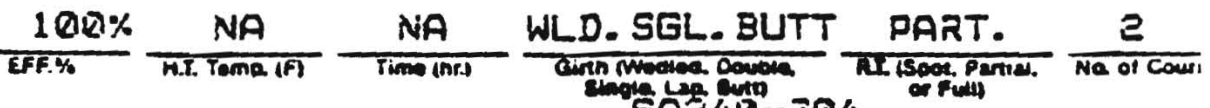

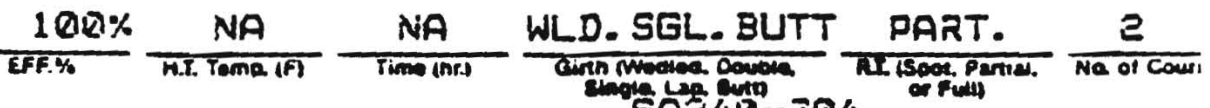
(b) Matl. Sค른 $40-304$

11. Supports: Skirt NA Lug NA Legs NA Other STRAPS 10eserioen

Attached ENDS WEIDED

(where and How

12. Remarks: Manufacturer's Partial Data Reports properly identified and signed by Commissioned Inspectors have been lurnished for the following items of the report:

NA

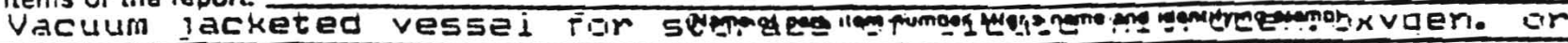
araon. Desian oressure is i06. Dasi. Inner vessei creca onlv ivoro oorts are ofuccea anc seaj wejoed.

\section{CERTIFICATE OF SHOP COMPLUANE}

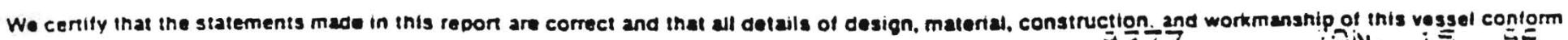

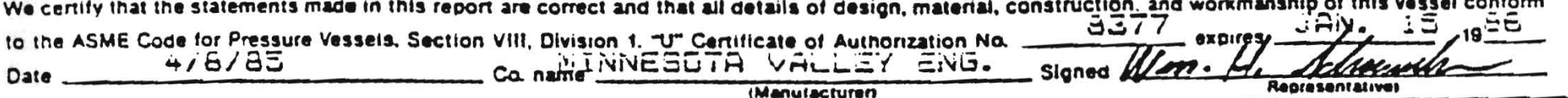

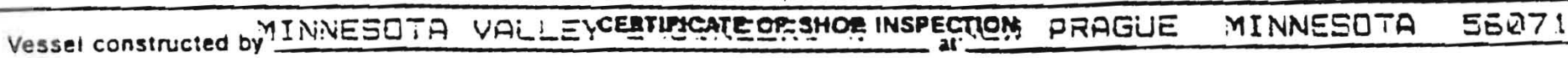
I, the undersigned. nolding a vatid commission issued by the Natlonal Board of Boiler and Pressure vessel Inspectors andor ine Slate or Provin and employed or. - 19 S5. und state that. to the best of

have inspected the component described in inis Manulacturers Data Repon on my knowtedge and beliet, the Manufacturep nas constructed this pressure vessel in accordance with ASME Code. Section viII. Oivision 1. By signing inis Cer. tificate neither the inspector nor his employer makes any warraniy, expressed or imptied. concerning ine pressure vesset described in this Manufacturef's Data feport. Fruthermora, netther ine inspector nor his employer snall be liable in any mannef for amy personal injury or properiy damage of a loss of any

Daie $5-1-85$ Signed commissions NB-3420 


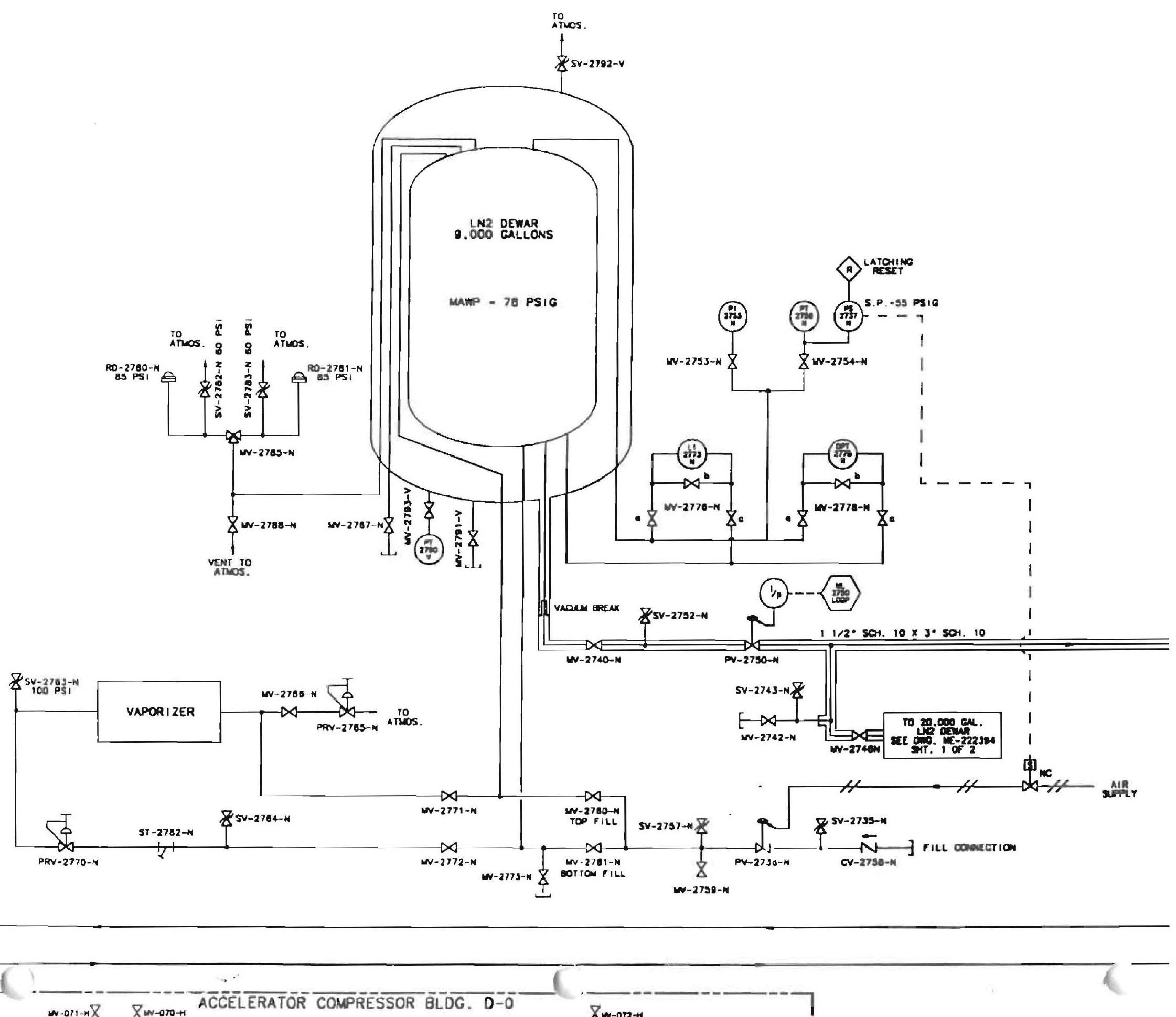




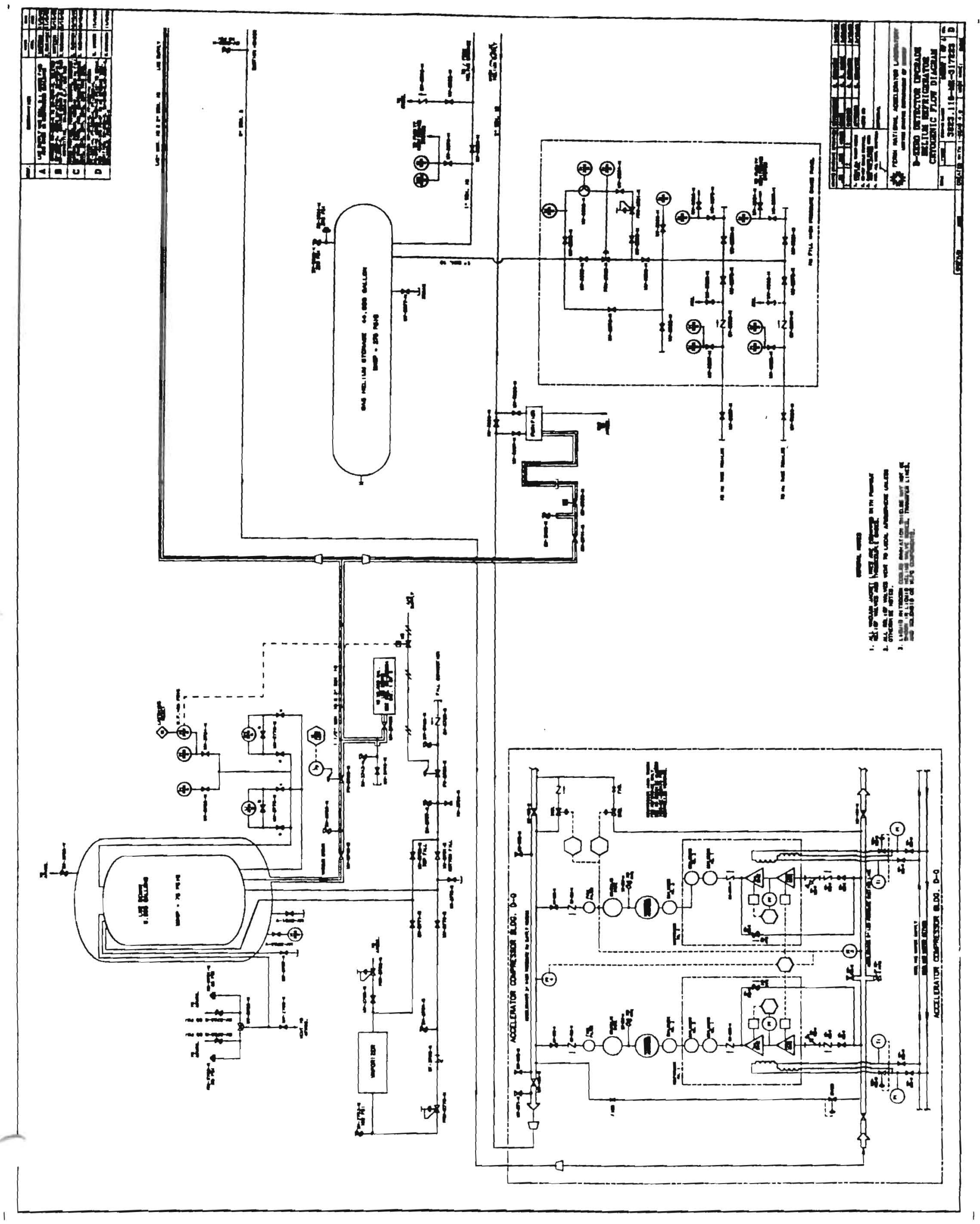


November 6, 1996

Revised January 13, 1997

Russ Rucinski

\section{LN2 Dewar Relief Summary}

Cases ( See Appendix A)

1.) Loss of vacuum.

2.) Fire.

3.) Pressure building coil failure.

4.) Pumping overfill.

5.) Filling a warm dewar

\section{Capacity Requirement}

$40.8 \mathrm{scfm}$ Air

62.2 scfm Air

82.3 scfm Air

Alternative method chosen

Alternative method chosen

ReliefCapacities (See Appendix B)

AGCO relief valve, Set Press. $=60$ psig

271.6scfm Air@78 psig

1" Fike Rupture disc, Burst Press. $=85$ psig

893 scfm Air@85 psig

Inlet Pressure Drop Calcs, (See Appendix C)

At maximum flow (Pressure building case), the pressure drop of inlet piping $=0.3 \mathrm{psi}$

Maximum vessel press. $=60.3$ psig $\ll 110 \%$ MAWP, ASME UG125, CGA met

Inlet losses $=.3 / 60=0.5 \%$ Set pressure $<3 \%$ Relief Set Pressure, API RP520 met

For Reference; $\quad$ MAWP of LN2 dewar $=78$ psig

$110 \%$ MAWP $=1.10(78+14.7)=102 \mathrm{psia}=87.3 \mathrm{psig}$

Relief set press. chosen to be approx. $70 \%$ RD burst pressure.

1/13/97 Revision: Loss of Vacuum and Fire case relief capacity requirements were 25.9 and 54.5 scfm respectively. 


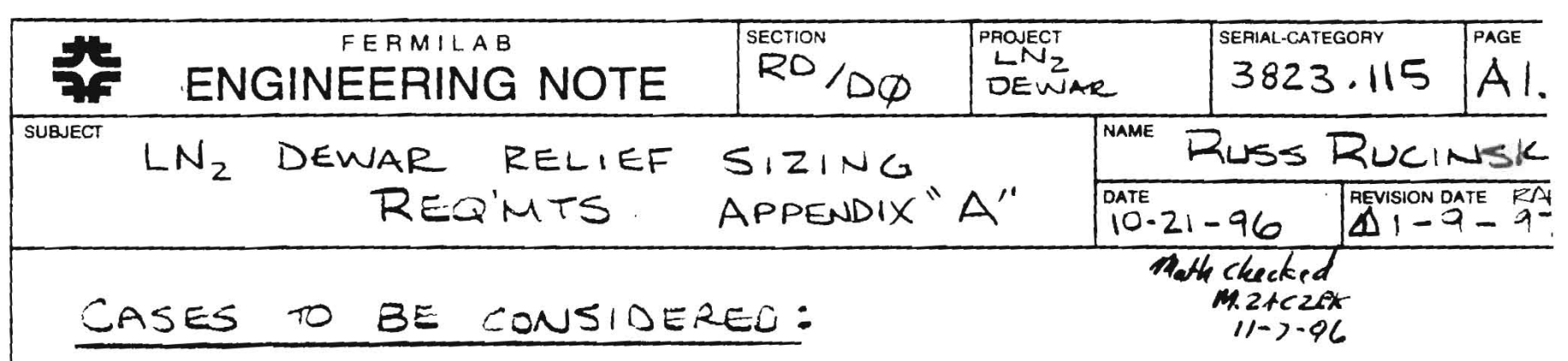

1.) LOSS of VACUUM

2.) FIRE

3.) PRESSURE BUILDING COIL FAILURE

4.) PUMPING OVERFILL

5.) filling a warm dewar

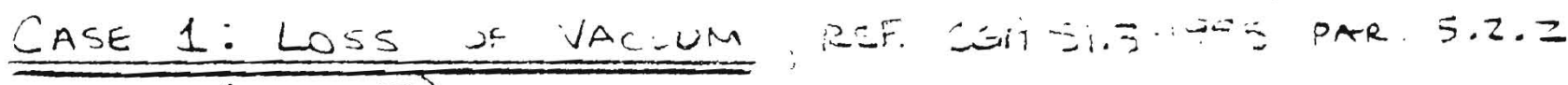

$$
\begin{aligned}
& Q_{a}=\frac{(590-T)}{4(1660-T)} F G ; \cup A \\
& \text { WHERE: } \\
& T=T_{\text {SAT }} \text { I FLOWRATING DRLSSURE } \\
& =T_{\text {sat }} \equiv(75+14.7)(10=102 \text { psia } \\
& =98.0 \mathrm{~K}=177{ }^{\circ} \mathrm{R}
\end{aligned}
$$

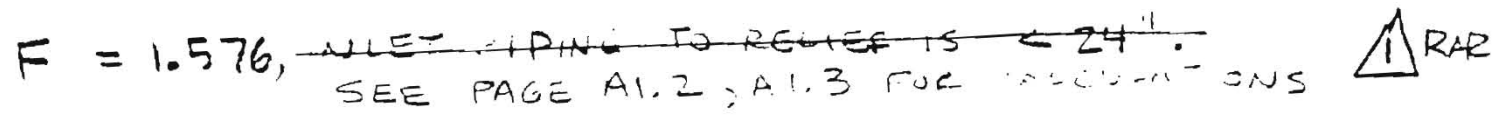

$$
\begin{aligned}
& G=0.2 \text { FROM GaLE } 1 .
\end{aligned}
$$

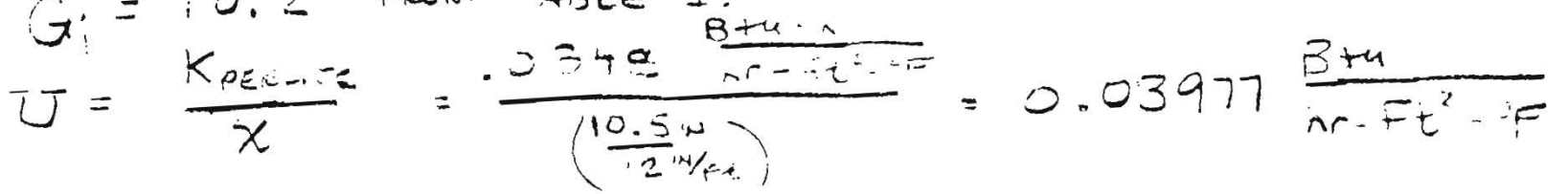

$$
\begin{aligned}
& A=(O A L+.19 .00)(00)(2.1416) \text { RE CGAS1.3:105 } \\
& A=\left(27.3 \mathrm{Ft}+19(1.0 \mathrm{Ft})(10 \mathrm{Ft})(3.4 .6)=918 \mathrm{Ft} \mathrm{F}^{2}\right.
\end{aligned}
$$

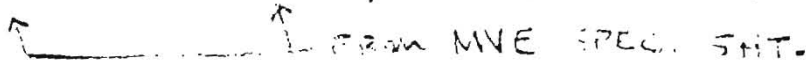

$$
\begin{aligned}
& Q_{a}=\frac{(570-177)}{4(1000-1.576}(10.2)(.0399)(918) \\
& Q_{1}=\geq 5 \\
& \hat{1} \\
& \text { ZRCF: 5U32.TA }
\end{aligned}
$$




\section{] Vertical Liquid Stations}

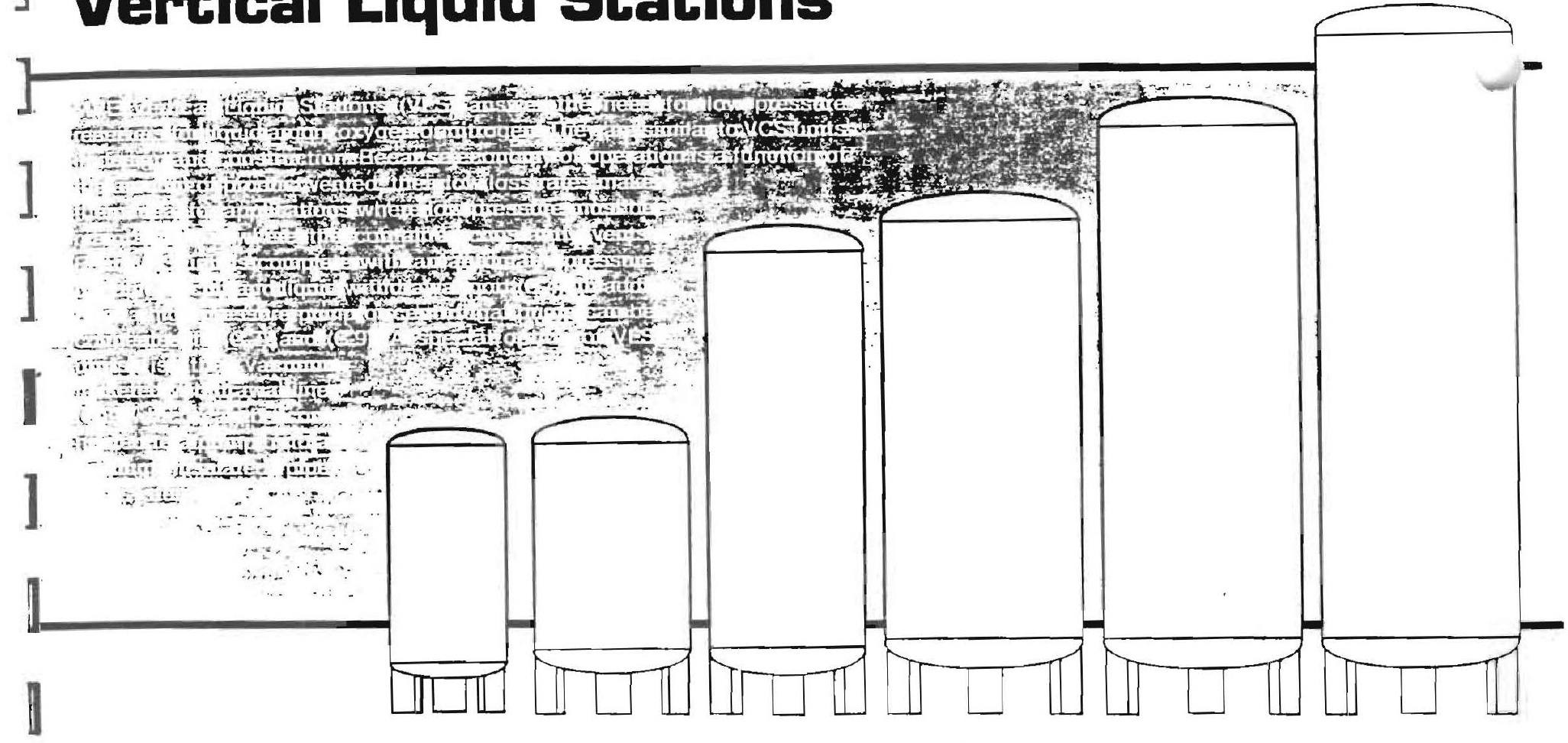

Two-year warranty

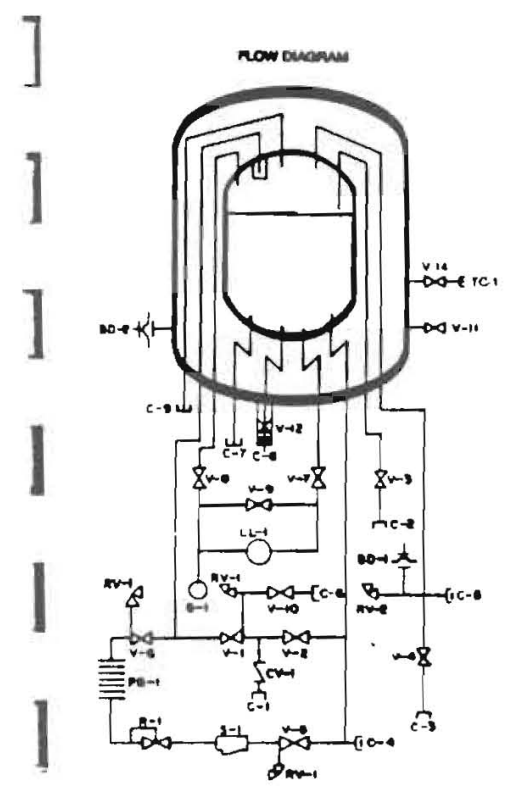

\section{SPECIFICATIONS}

\begin{tabular}{|c|}
\hline MODEL \\
\hline Diameter (inches) . . . . . . . . \\
\hline Height (inches) . . . . . . . . \\
\hline Gross Capacity (gallons) . . . . \\
\hline Net Capacity (gailons) . . . . . \\
\hline $\begin{array}{l}\text { Equivalent Capacity of Gas } \\
\text { Oxygen (SCF at NTP) } \ldots \\
\text { Nitrogen (SCF at NTP) . . . } \\
\text { Argon (SCF at NTP) . . . }\end{array}$ \\
\hline Design Pressure (PSIG) . . . . \\
\hline $\begin{array}{l}\text { Weights } \\
\text { Tare (ibs.) ... . . . . . . } \\
\text { Loaded Oxygen (ibs.) } \ldots \ldots \\
\text { Nitrogen (Ibs.) } \ldots \ldots \ldots \ldots \\
\text { Argon (Ibs.) } \ldots \ldots \ldots \ldots \ldots\end{array}$ \\
\hline $\begin{array}{l}\text { Normal Evaporation } \\
\text { Rate } \% \text { /Day LOx . . . . . . . }\end{array}$ \\
\hline
\end{tabular}

$\begin{array}{cc}1,500 & 3,000 \\ 66 & 96 \\ 180 & 185 \\ 1,629.5 & 3,062.0 \\ 1,458.5 & 2,980.0 \\ & \\ 167,862 & 343.000 \\ 135.792 & 277.468 \\ 164.070 & 335.250 \\ 50 & 50 \\ & \\ 5,000 & 10.650 \\ 18.894 & 39.040 \\ 14.837 & 30.750 \\ 21.961 & 45.307\end{array}$

6,000
96
309
$6,095.0$
5.962 .5
686.284
555.168
670.781
50

.40

.17

18,900

75.705

59.117

88.244

.15

$\downarrow_{900}$
9,000
120
328
9.200
9.000
1.035 .500
838.000
1.012 .000
78
24.900
110.650
85.600
129.550

.10

$\begin{array}{cc}11,000 & 13,000 \\ 120 & 120 \\ 387 & 444 \\ 11.300 & 13.300 \\ 11.000 & 13.000 \\ & \\ 1,266.100 & 1,496.000 \\ 1.024 .210 & 1,210.000 \\ 1.237 .500 & 1.462 .000 \\ 75 & 72\end{array}$

30,000

134,800

104.200

157,900

35.400

159.265

122.100

186.570

.10

I

\section{NOMENCLATURE}$$
1
$$

V-1 VALVE, TOP FILL

V-2 VALVE, BOTTOM FILL

V.3 VALVE, FULL TAYCOCK

V-4 VALVE, VENT

V.5 VALVE, PRESSURE BUILDING

V.6 VALVE, PRESSURE BUILDING AND ECONOMIZER ISOLATION

V-7 VALVE, LIQUID PHASE (HIGH)

V-8 VALVE, GAS PHASE (LOW)

V.9 VALVE, EQUALIZATION

V-10 VALVE, DRAIN

V.11 VALVE, EVACUATION

$\checkmark-12$ VALVE, VACUUM JACKETED (OPTIONAL)
V-14 ISOLATION VALVE, VACUUM TUBE

CV-1 CHECK VALVE, FILL

R-1 REGULATOR, PRESSURE BUILDING

RV.1 RELIEF VALVE, LINE

RV-2 RELIEF VALVE, TANK

BD-1 BURST DISC, TANK

BD-2 BURST DISC, ANNULAS

PB-1 PRESSURE BUILDING COIL

LL-1 GAUGE, LIQUID LEVEL

G-1 GAUGE, PRESSURE

S-1 STRAINER

C-1 CONNECTION, FILL

C-2 CONNECTION, FULL TRYCOCK

C-3 CONNECTION, VENT
C-4 CONNECTION, LIQUID WITHDRAWAL (CAPPED)

C-5 CONNECTION, VENT (CAPPED)

C-6 CONNECTION, DRAIN

C.7 CONNECTION, PUMP

C-8 CONNECTION, FIELD JOINT (OPTIONAL)

C.9 CONNECTION, PUMP VENT TC-1 VACUUM TUBE 


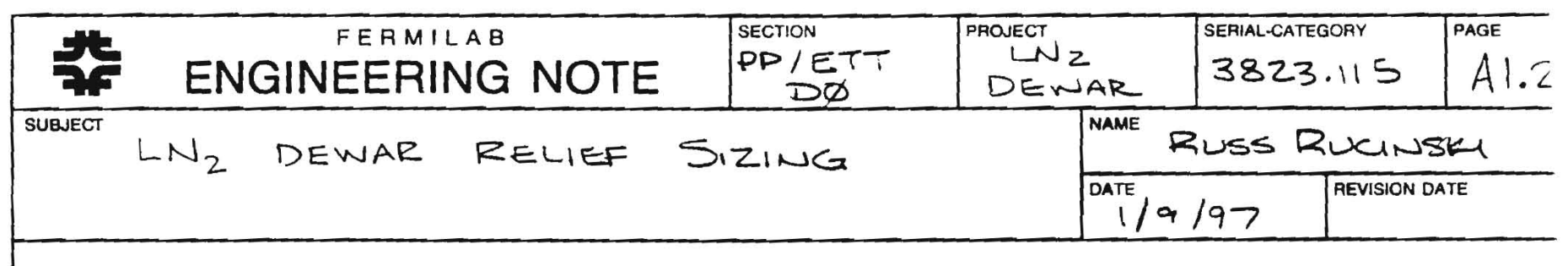

- Determination of "F" correction factor per cha si.3-1995 dar. 5.1.4

$$
F=\sqrt{\frac{Z_{i} T_{i}}{Z T}}=\sqrt{\frac{P_{i} \nu_{i}}{P_{\nu}}}
$$

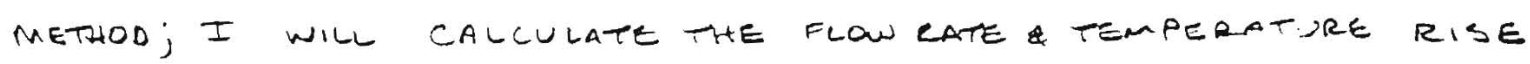
in the relief piping. Follow cha procedure.

Loss of VACUUM CASE:

STEP 1

$$
\begin{aligned}
& \text { ASSUME } F=1.0 \text { FOE HOW. THEN BU LEA } 3.2 .2 \text {; } \\
& Q_{a}=\frac{(590-T)}{4(1660-T)} \text { FilA }=25.9 \text { sCAM air }
\end{aligned}
$$

EONUELTING is MASS Flow of Flow in's MEOAN:

$$
\begin{aligned}
& T=\frac{Q_{\text {ae }}}{\text { is.3 }} \sqrt{\frac{M}{\equiv T}}=\frac{(25.9 \text { scFma.e })(356)}{18.55} \sqrt{\frac{28.0: 4}{(.82)\left(177^{\circ} \mathrm{R}\right)}} \\
& \frac{P}{P_{c}}=\frac{.02 \text { pis }}{493.134 \text { psia }}=0.207 \quad \frac{T}{T_{c}}=\frac{177^{\circ} \mathrm{R}}{27.66^{\circ} \mathrm{R}}=0.78
\end{aligned}
$$

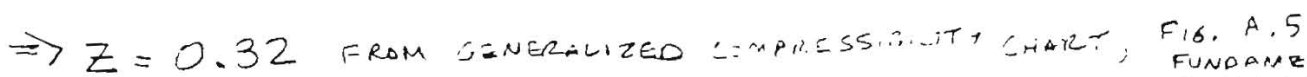

$$
\begin{aligned}
& \text { FUNDAMENTAlS vT } \\
& \text { ELASSICATL TITERM.O } \\
& W=221 \mathrm{ibm} / \mathrm{hr} \mathrm{N}_{2} \text {. } \\
& 3^{\text {rd }}=0 \cdot 1995
\end{aligned}
$$

$$
\begin{aligned}
& \text { s-1 } 2 \\
& T_{i}=590-\frac{590-T_{S}}{\left.-\frac{50 L}{W C P}\right]} \quad T_{S}=177^{\circ} \mathrm{R} \quad D=1.70^{\prime \prime} \quad \text { L\#40 FEET } \\
& T_{i}=590-\frac{590-i 77}{\Delta\left[\frac{.55(1.4)(\Delta 0)}{2(21(-.9 .034)}\right.}
\end{aligned}
$$

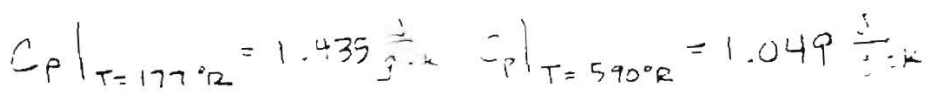

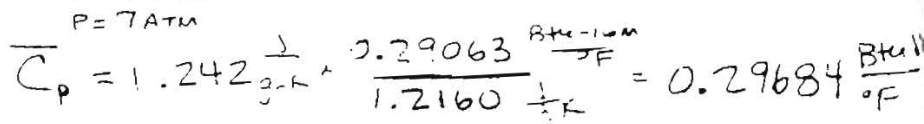

$$
\begin{aligned}
& r=5 ? 2 \\
& \begin{array}{l}
\text { VALUES FEM } \\
\text {-ABLE } 5 \text { GoA }
\end{array} \\
& T \text { For Fund isoper-ies }=\frac{372^{\circ} R-177^{\circ} R}{2}=275^{\circ} R
\end{aligned}
$$




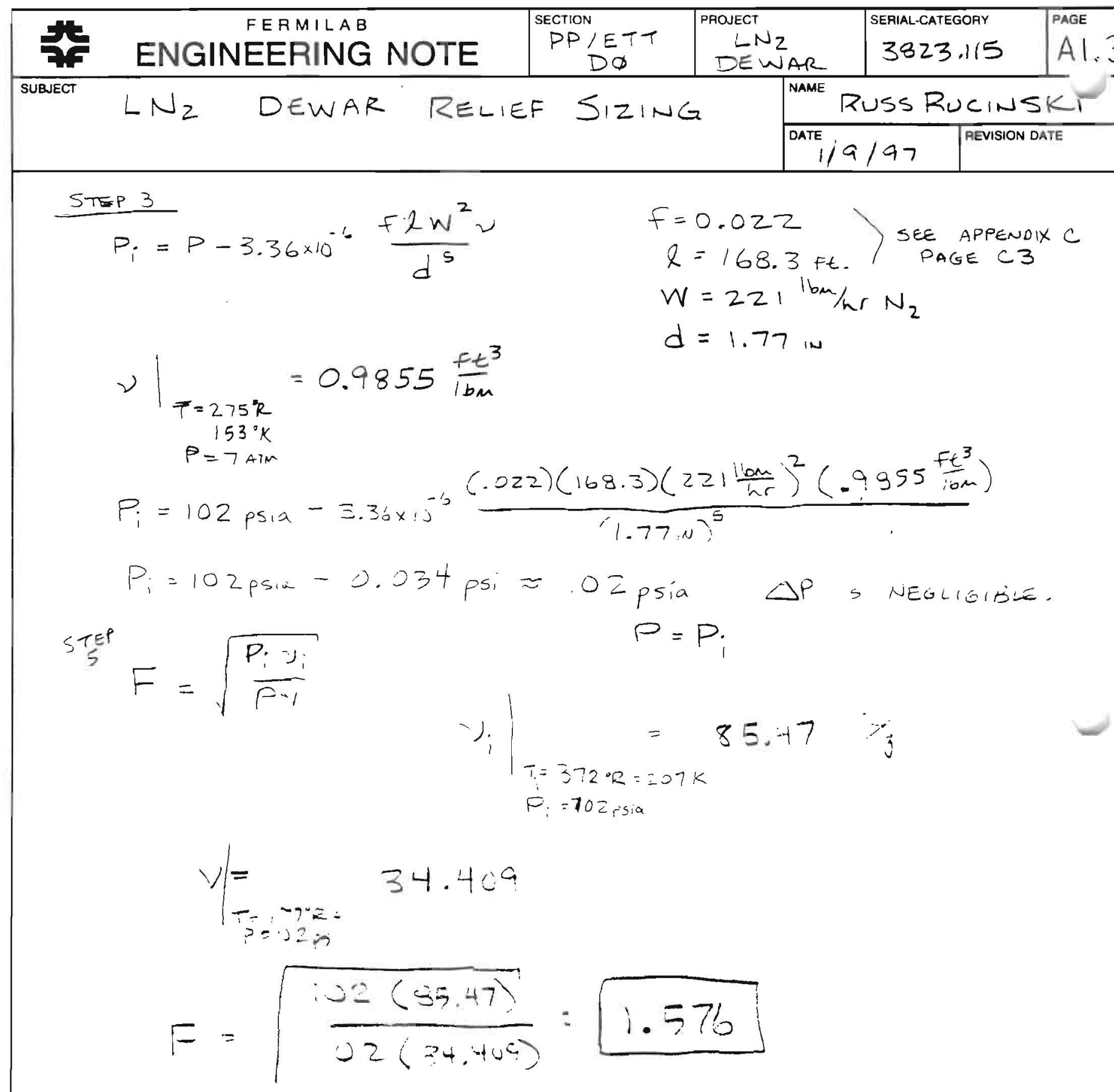




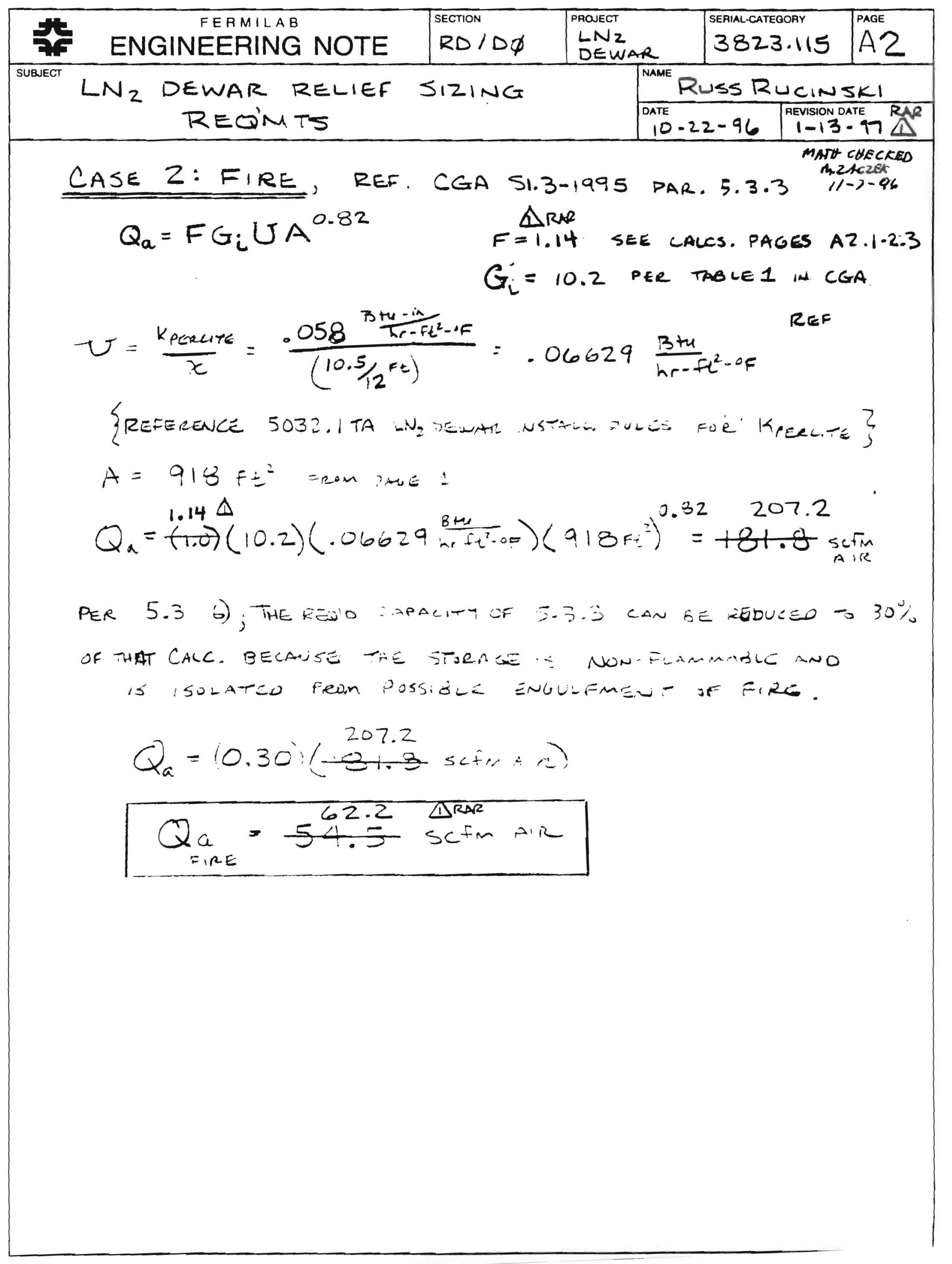




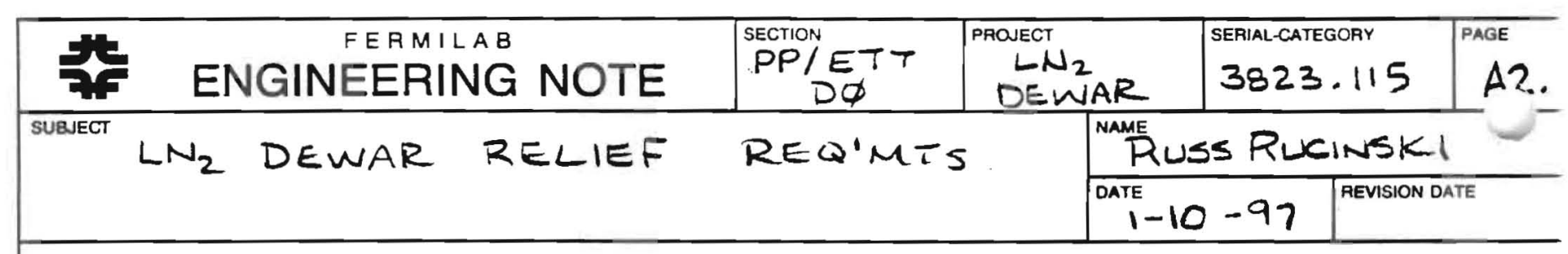

- determination of "fo" correction factor per coasi.3 - 1995 par. 5.1. 4

I. WILl aPply the same method as dONE fOr loss of VAcuUm, pages $A_{1} .2, A_{1} .3$. ReFerence those paves FOR LONGHAND ACCOUNT OF STEPS.

FIRE CASE

STEP 1

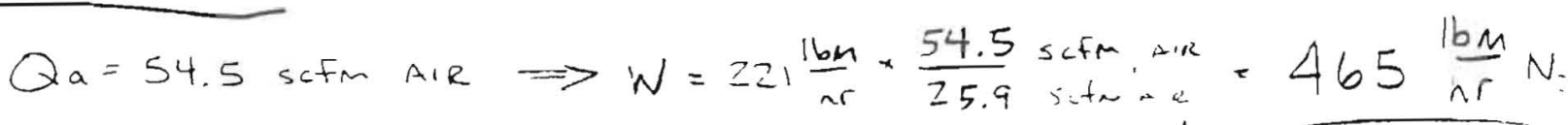$$
\text { STEP } 2
$$

$$
\begin{aligned}
& T_{i}=21+5-\frac{2145-T_{s}}{e^{\left[\frac{5.24 D L}{W C_{p}}\right]}}=2145-\frac{2145-i 7 i}{e^{\frac{5.24(1.9)(40)}{465(.29634)}}} \\
& T_{i}=I 0 \equiv 5 \quad \cdot R
\end{aligned}
$$

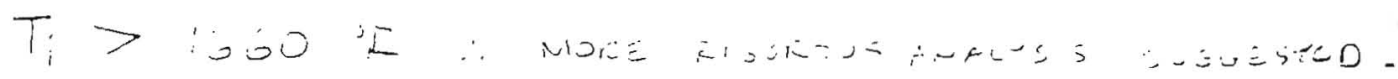

WHILE LIQUID REMAINS W THE TANK THE InNER WALl temperature ian be ASSUMED T BE TSAR $P$ P $P=121 \%$ MANY $=112 P \leq A=7.6 \mathrm{ATM}$ FR JUL NED: E:IND $=E$ 'TOLE

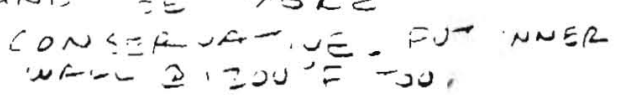

$T_{5,1}=: 80 \%$

JESSE- WAR WA :L
$<10,5:>$

MODE: AS:

$1.9 " 1$ PERLITE

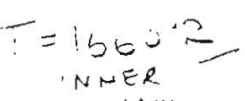

4. 3

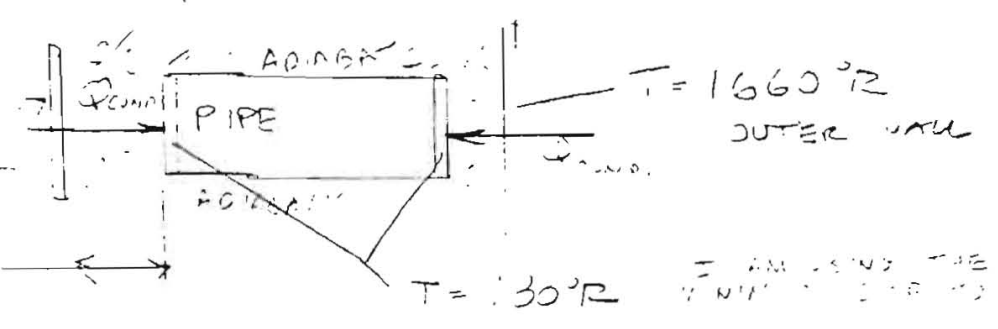

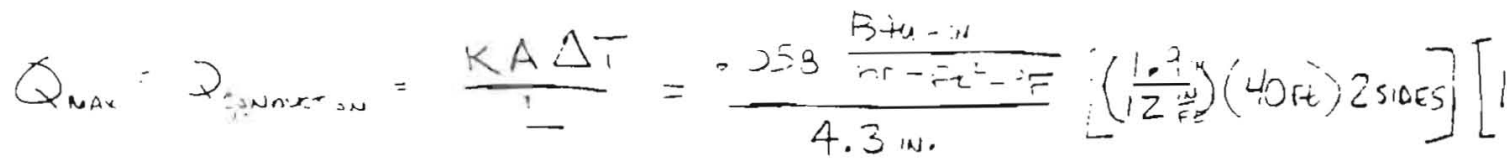

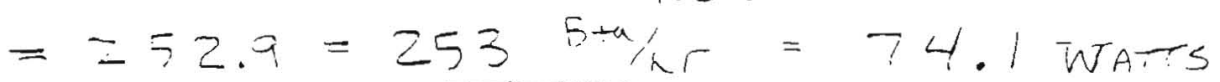




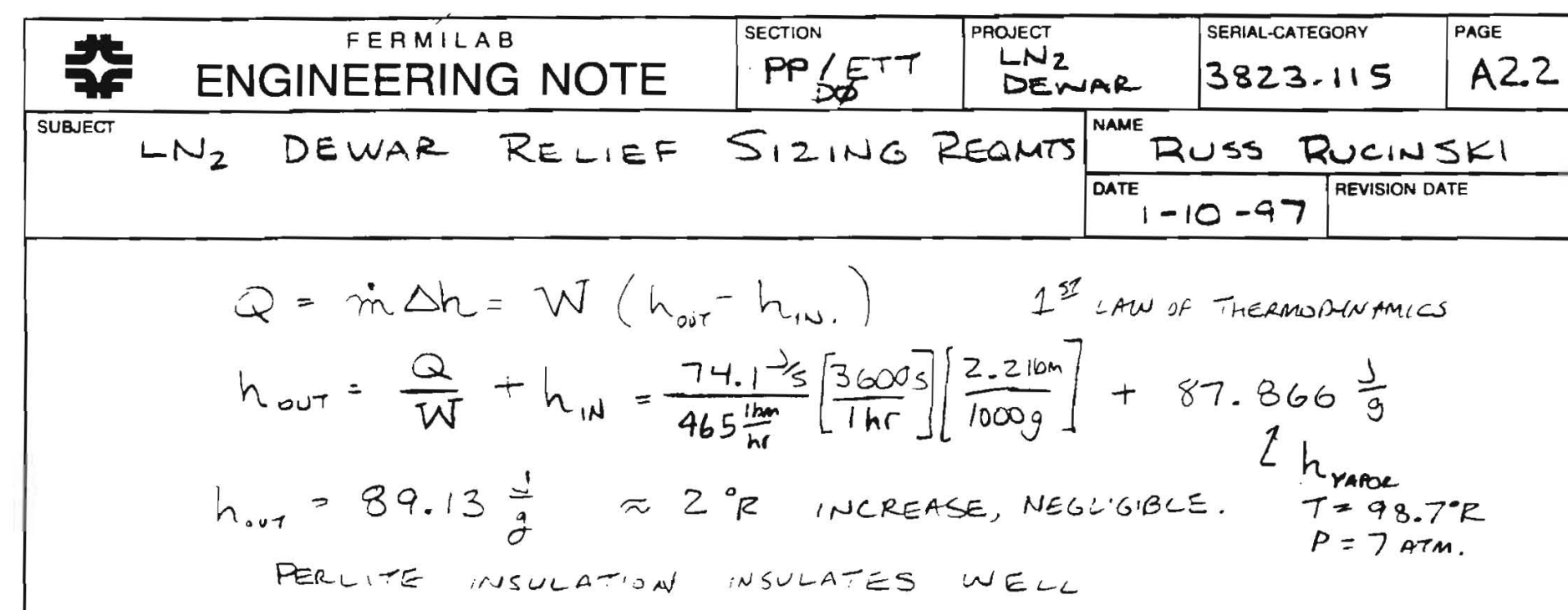

Alternative check of the heat flux number:

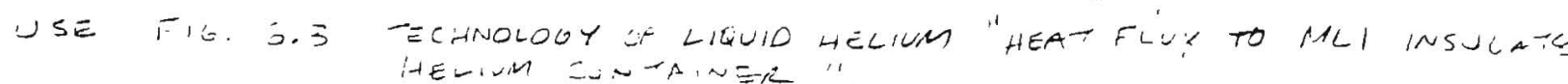

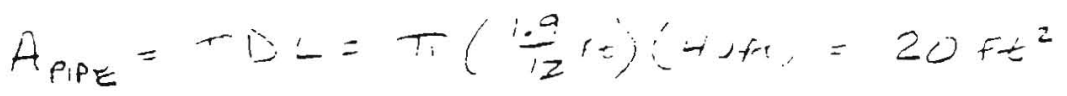

$$
\begin{aligned}
& \text { ChoOSE FIRE: 4.j" SI HEAT FLUV }=20.000 \frac{\mathrm{BH}}{\mathrm{hr}} \text {. }
\end{aligned}
$$

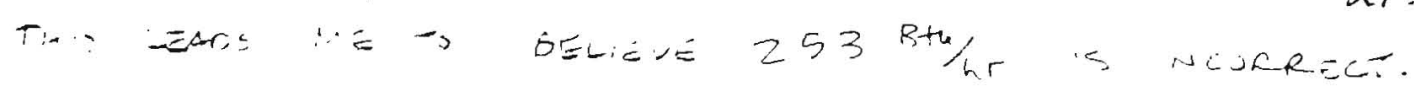

CONSIDER:

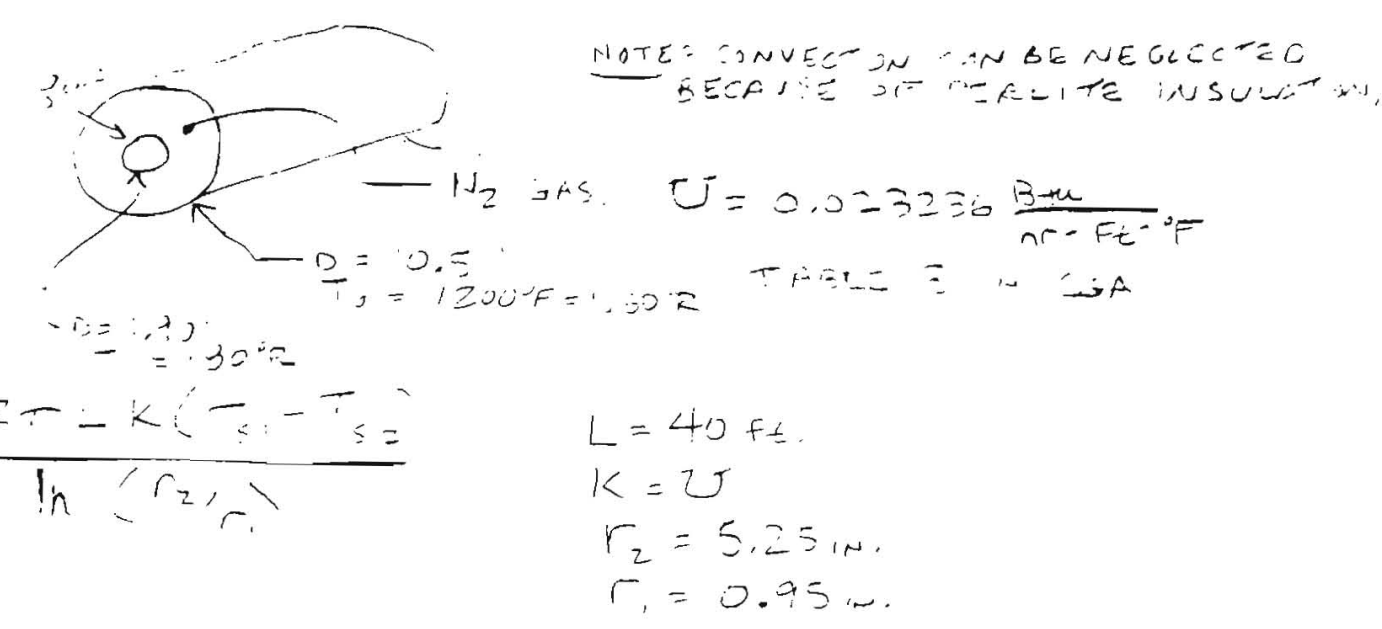

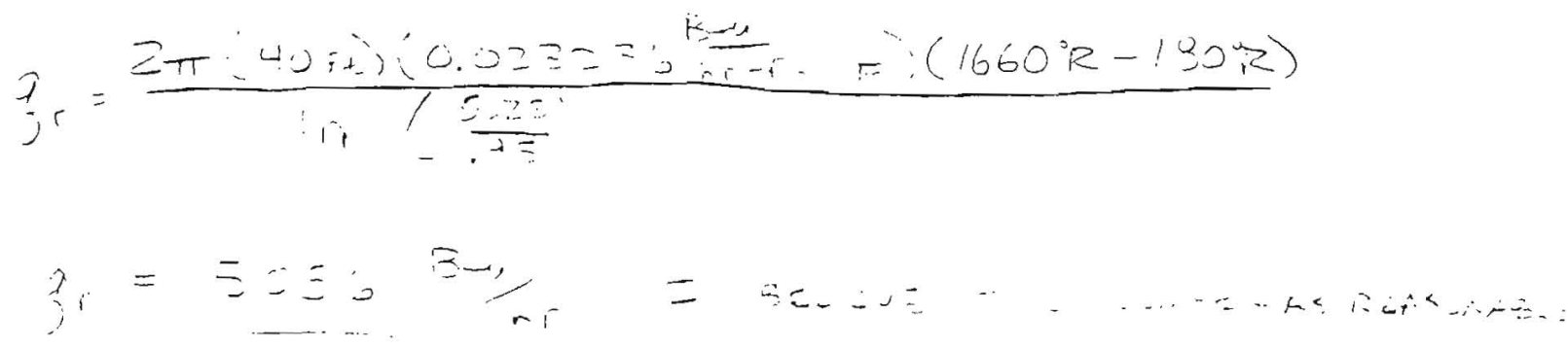

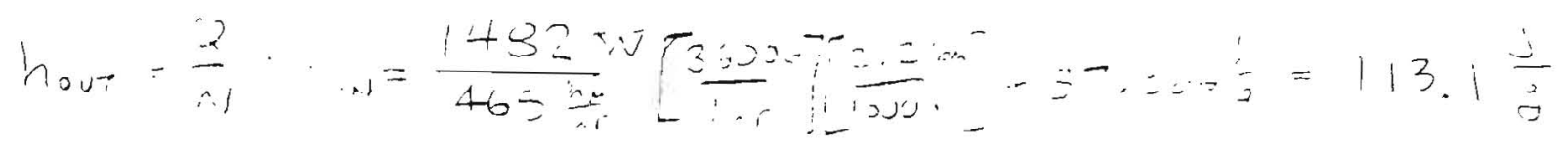




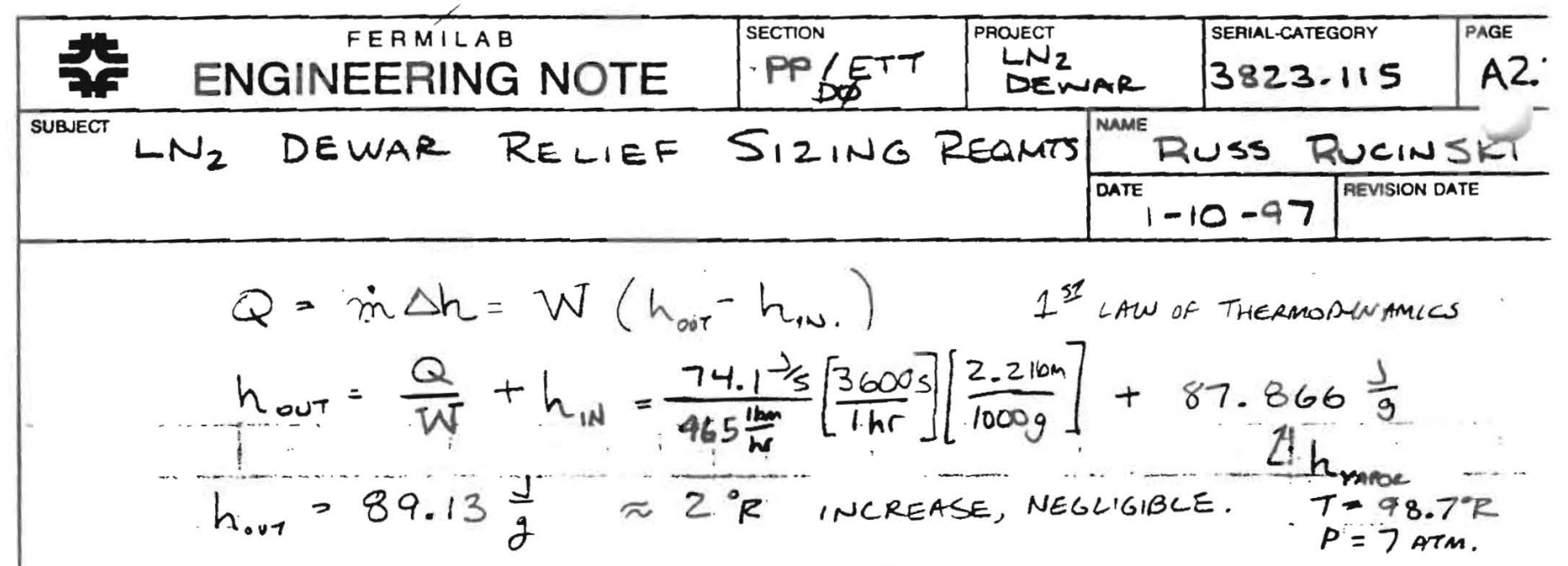

perlite insulation insulates well

Alternative check of the heat flux number;

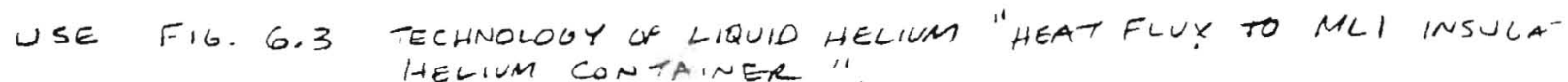

$$
A_{\text {PIPE }}=\pi D L=\pi\left(\frac{1.9}{12} \mathrm{Ft}\right)(H U \mathrm{FH})=20 \mathrm{Ft}^{2}
$$

CHOOSE FIRE, 4.5" SI HEAT FLUY $=20,000 \frac{\mathrm{BH}}{\mathrm{hr}}$. Titis leads ME to believe $253 \mathrm{Bth} / \mathrm{hr}$ is incorrect.

CONSIDER;

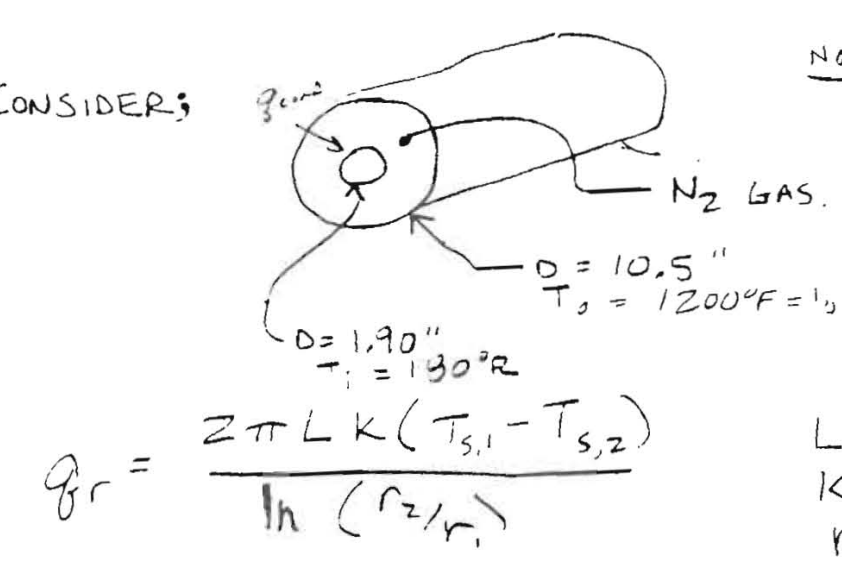

NOTE CONVECTION GAN BE NEGLECTED BECAUSE DO PERLITE INSULATION

$$
\begin{aligned}
& U=0.023236 \frac{B+\mu}{n F^{-}{ }^{\circ} F} \\
& \text { TABLE } 3 \text { i G GA }
\end{aligned}
$$

$$
\begin{aligned}
& L=40 \mathrm{ft} . \\
& K=U \\
& r_{2}=5.25 \mathrm{~N} . \\
& r_{1}=0.95 \mathrm{~N} .
\end{aligned}
$$

$$
\begin{aligned}
& q_{r}=\frac{2 \pi(40 \mathrm{ft})\left(0.023236^{\frac{B-u}{0 r}-F+\cdots}\right)\left(1660^{\circ} R-180 \mathrm{R}\right)}{\ln \left(\frac{5.25}{.75}\right)}
\end{aligned}
$$

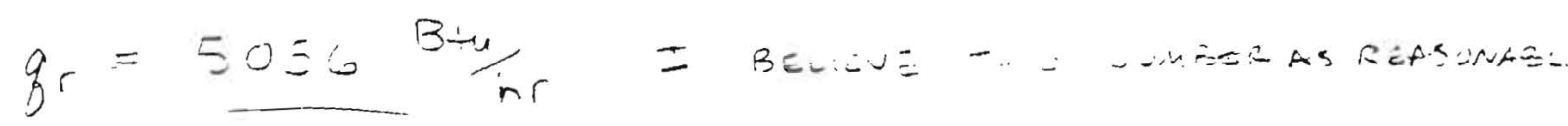

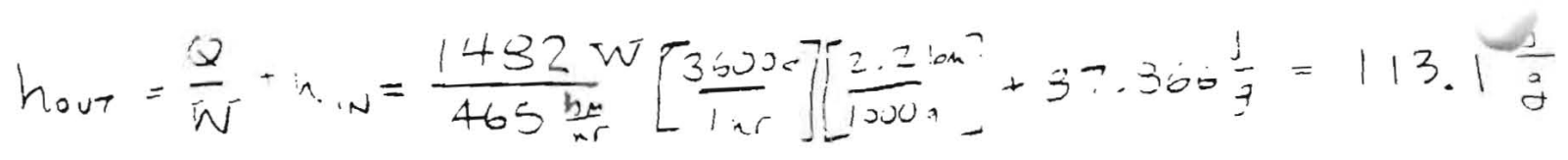

From therm properties tables $P=T_{\text {ATM }}, T_{i}=118 \mathrm{~K}$

$$
T_{i}^{i}=212.4^{\circ} \mathrm{R}
$$




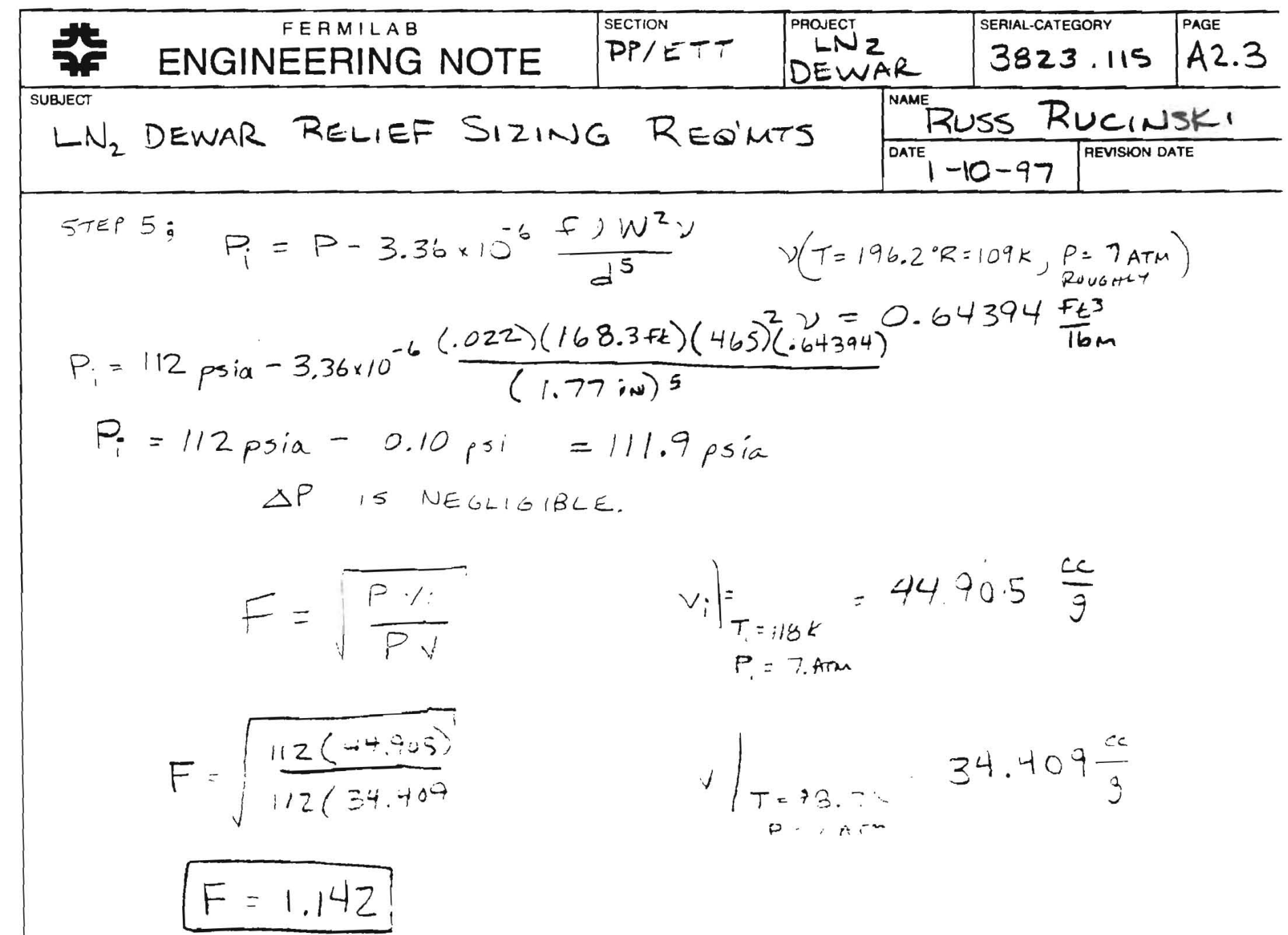




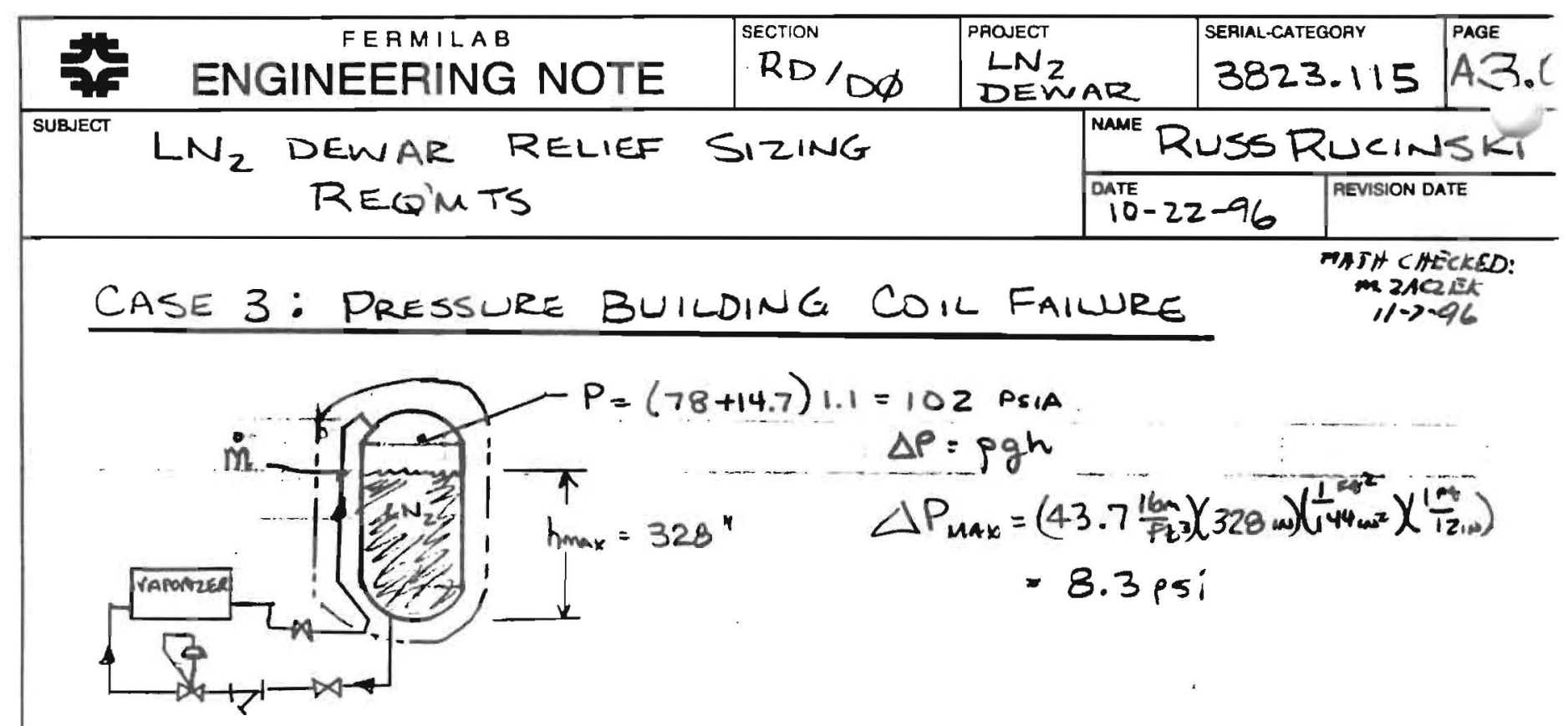

Determine the required relief valve capacity for the case of wide open inlet and outlet valves to the vaporizer.

THE SOLUTION TO THIS PROBLEM CAN BE BOUNDED BY THE LESSER OF Z MODELS;

1 II MODEL: CONSIDER the vaporizer as an INFINITE SOURCE OF HEAT. THE MASS FLOW RATE IS THEN LIMITED BY THE INLET E OUTLET PIPING SIZE AND THE MAXIMUM HEAD OF 8.3 PSi. ASSUMING SATURATED LIQUID THRU THE VAPORIZER INLET PIPING AND SATURATED VAPOR THRU the outlet, the result is:

$$
\dot{m}_{\text {MODEL 1 }}=1564 \mathrm{lb} / \mathrm{hr} \mathrm{Nz}
$$

2" MODEL : CONSIDER THE PIPING TO BE SUFFICIENTLY LaR oE SUCh that the mass flow rate :S LIMITED BY THE HEAT INPUT CAPACITY of the vaporizer. The result is;

$$
\begin{aligned}
& \dot{M} \text { MODEL } \\
& Q_{a}=82.3 \mathrm{SCFM} \mathrm{AIR}
\end{aligned} \text { SAT. } \mathrm{N}_{2} \text { VAPOR }
$$

THE RAW CALCS: For BOTH MODELS Follow. 
PRESS BLDG. COIL FAILURE CASE 3

RAN CALLS.

R.Rucinsic 10/22/96 3.1

PB COL FAILURE

MATH CHECKS

SCENARIO, PRV-2770-N FAILED FULL OPEN $11-7-96$ SAM SPRING SNAPPED OR OPERATOR ERROR'J

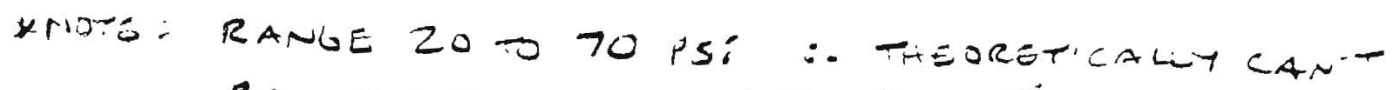
BE SET TO $>$ MAW $=78$ riGi

DRIVING FORCE FOR MASS FlOW IS LIQUID HEAD.

$$
h=328_{\mathrm{in}} \text {. }
$$

VAPORIZEr

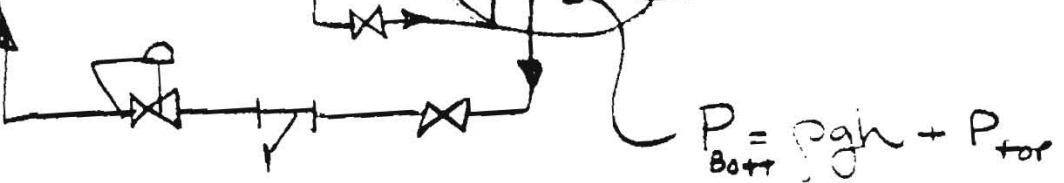

$$
\begin{aligned}
& \rho_{\text {LIQ }}(102 \text { psia: Tart })=.70^{\mathrm{g} / c c}=43.70^{\mathrm{lb}} / \mathrm{Ft}^{3} \\
& \Delta P=\left(43.7 \frac{1 \mathrm{~b}}{F_{t^{3}}}\right)(328 \mathrm{NN})\left(\frac{1 F_{t^{2}}}{144 N^{2}} Y \frac{1 F t}{12 \mathrm{~N}}\right)=8.3 \mathrm{psi}
\end{aligned}
$$

- max. flow late can be conservatluecy calculated BY ASSUMing SAT. LIVUID. TAHOLLAH VAPORIZE L INLET F,OU-DET PIPING WiTH 3.3 PSi $\triangle P$.

SAT. apure ?

PHYSICAL PIPING SKETCH

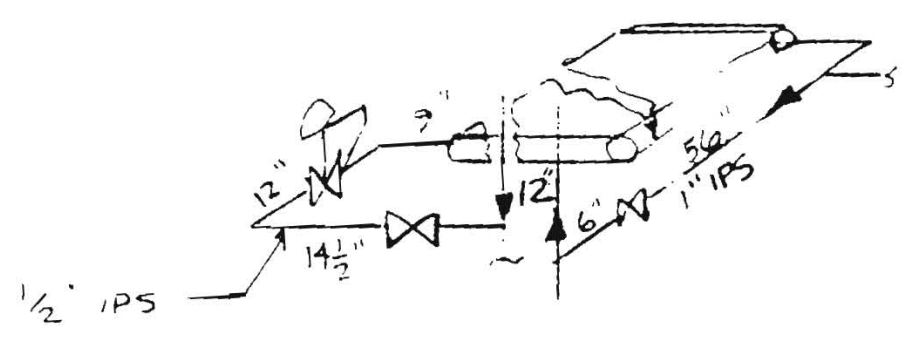

- calculate equivalent piping length for 1/2 lips size. INLET PIPE $L=12+14 \frac{1}{2}+12+9=47.5=4 \mathrm{FE}$.

inlet valves $K=340 f_{T}$ each ale

$I_{-}=\ldots$ 
PRESS BLDG. COLL FAILURE CASE 3

Russ Rucinski 10/22/96 3.2

$$
L=\frac{K D}{F}=\frac{2(340)(.027)\left(\frac{.622}{12}\right)}{.027}=\frac{35.2}{1 / 2 " \text { PIPE }}
$$

MATH CHECKED

M. $2 x 2$ \&

INLET TEE +2 ELBOWS $\Rightarrow K=60 \mathrm{Ft}+2\left(12 \mathrm{Ft}_{t}\right)=84 \mathrm{f}_{T}=(17.6 \mathrm{Ft}) \times \frac{84}{340}=4.35 \mathrm{Ft}$. OUTLET PIPING $L=56+6+12=74^{\prime \prime}=6.17 \mathrm{FE}$ " PIPE *

$$
K_{\text {Puff }}=\frac{F E}{D}=\frac{.023(6.17)}{\left(\frac{1.049}{12}\right)}=1.6225 \text { For " size }
$$

OUTLET

$$
\begin{aligned}
& \text { OUTLET: } 2 \text { TEES }+1 \text { ELbOW }=20 \mathrm{ft}_{t}+20 \mathrm{f}_{t}+60 \mathrm{ft}_{t}=100 \mathrm{ft}_{\mathrm{t}} \\
& K=100(.023)=2.3 \text { FOR 1" size } \\
& \sum K_{\text {purer }}=10.12 \\
& L=\frac{K D}{F}=\frac{10.12\left(1 . \frac{049}{12}\right)}{.223}=38.46 \mathrm{rz}
\end{aligned}
$$

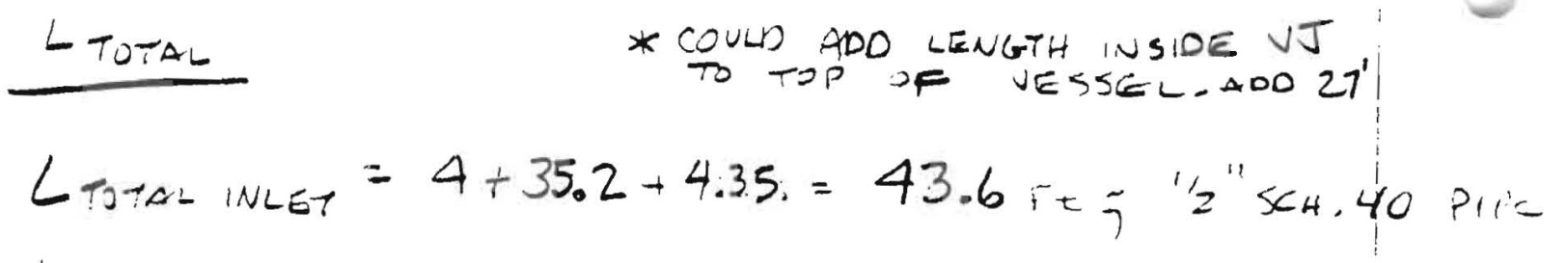

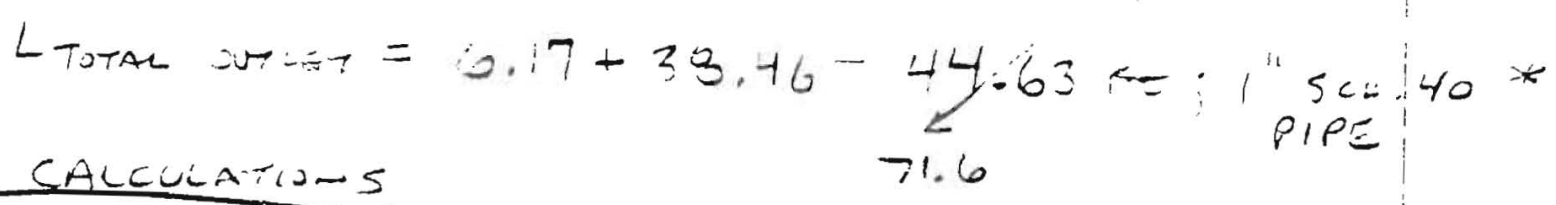

$\triangle P P$ calcuiatia-s

$$
\begin{aligned}
& f_{\tau}=.027 \text { for } 1 / 2 \text { " } \\
& F_{\tau}=.023 \text { for 1" }
\end{aligned}
$$

AND GHELK Re

$$
\begin{aligned}
& \text { iN GENL } \triangle P=3.36 \times 10^{-6} \frac{F L W^{2}}{\rho d^{9}} \\
& \Delta P_{\text {ITAL }}=3.36 \times 10^{-6}\left[\frac{F_{1} L_{1} W^{2}}{\rho_{l} d_{1}^{5}}-\frac{F_{2} L_{2} W^{2}}{\rho_{g} d_{2}^{5}}\right]
\end{aligned}
$$

Solving For W:

$$
W^{2}=\frac{\triangle P_{\text {TOTAL }}}{3.36 \times 10^{-6}\left[\frac{F_{1} L_{1}}{P_{\ell O_{1}^{j}}}+\frac{F_{2} L_{2}}{\left.P_{g d_{2}}\right]}\right.}
$$




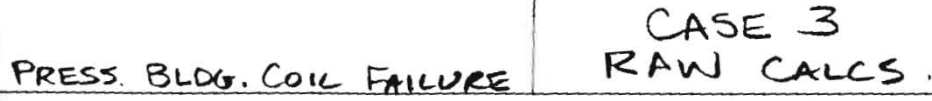

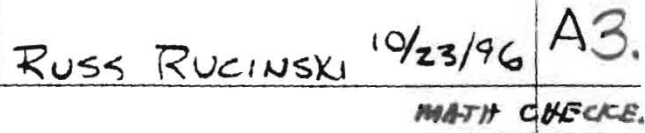

values; $\Delta P_{\text {Tut al }}=8.3$ psi

M. $2 x, 265$

$$
\begin{aligned}
& F_{1}=.027 \quad F_{2}=.023 \\
& L_{1}=43.6 \quad L_{2}=44.6 \\
& 71.6 \mathrm{Ft} . \\
& \rho_{l}=43.7 \frac{\mathrm{lb}}{\mathrm{ft}^{3}} \quad \rho_{g}=.02884 \frac{\mathrm{g}}{\mathrm{c} c}=1.8 \mathrm{lb} / \mathrm{ft}^{3} \\
& d_{1}=.622 \mathrm{in} . \quad d_{2}=1.049 \mathrm{in} \text {. } \\
& W^{2}=\frac{8.30 \text { es }}{3.36 \times 10^{-6}}\left[\frac{.027(43.6)}{43.7(.622)^{5}}+\frac{.023(44.6)}{1.8(1.049)^{5}}\right]^{-1} \\
& W=\frac{1564.2}{16} \mathrm{~kg} / \mathrm{hr} \quad \mathrm{Nz} \\
& \Delta P_{1}=3.36 \times 10^{-6} \frac{(.027)(43.6)(1562.2 .5)^{2}}{43.7(.622)^{5}}=2.38 \rho 5 i \\
& \Delta P_{2}=8.3-2.4=\underline{5.9} \text { ps: } \\
& \text { W }=(1829.5) 1564.2 \quad 2.14 \times 10^{5} \\
& \operatorname{Re}_{1}=6.31 \frac{\omega}{d \mu}=6.31 \frac{(.829 .5}{(.622)\left(755.9 \times 10^{-6} \frac{g}{\mathrm{~cm}-3)} \frac{100 \mathrm{cp}}{\left(\frac{g}{c_{\mu}-3}\right)}\right.}=2.5 \times 10 \\
& \frac{\varepsilon}{D}=\frac{.00015}{\frac{.622}{12}}=.0029 \\
& \Rightarrow F_{1}=.026 . \\
& \operatorname{Re}_{2}=6.31 \frac{\mathrm{N}}{d \mu}=6.31 \frac{18 \times 29.5^{150 t .2}}{(1.049)\left(70.79 \times 10^{-6}\right)(100 \mathrm{Cp})}=\frac{1.33 \times 10^{6}}{1.55 \times 10^{6}} \\
& \varepsilon_{D}=\frac{.00015}{\frac{1.044}{12}}=.0017 \\
& \Rightarrow F_{2}=.023
\end{aligned}
$$

FRICTION FALTORS ARE O.K. MAXIMUM FlOW RATE CALL. IS OK

$$
W=1564 \mathrm{lb} / \mathrm{hr} \quad \mathrm{N}_{2}
$$


PRESS. BLOG. COL FAILURE

CASE 3

RUSS ZXCINSK1 O/23R6 AB:

- check amt. of heat redid to change the phase from liquid to gas.

$$
\begin{aligned}
& Q_{\text {CEO'O }}=\bar{m} h_{f g}=1564 \frac{16}{h r}[87.866-(-75.578): \\
& \times \frac{1 h r}{36005} \quad P=7 A T M, N B S \cdot T N \cdot 1025 \text { ThC } \\
& Q_{\text {REOSD. }}=32,276 \text { WaTtS. }
\end{aligned}
$$

THIS PROBABLY EXCEEDS AMOUNT pOSS bl by vaPoRIZER, $\therefore$ aT THIS flow rate; outlet of vaporizer wOULD be 2 phase; $\triangle P$ by quid head goes away.

conservative estimate vaporizer capacity:

SURFACE AREA OF FINS;

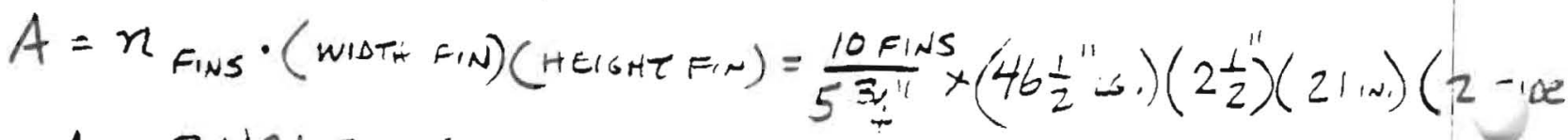

$$
\begin{aligned}
& A_{\text {INS. }} 8491.3 \mathrm{in}^{2}=5.48 \mathrm{~m}^{2}
\end{aligned}
$$

$$
\text { SAM } \begin{aligned}
h & =10 \frac{\mathrm{W}}{\mathrm{m}^{2} \mathrm{~K}} \quad \text { RANGES CON } \quad 2-25 \mathrm{~W} / \mathrm{m}^{2} \mathrm{~K} \\
\Delta T & =293 \mathrm{~K}-99 \mathrm{~K}=194 \mathrm{~K} \\
q^{\prime \prime} & =h \Delta T=10 \frac{\mathrm{w}}{\mathrm{m}^{2} \mathrm{~K}}(194 \mathrm{~K})=1940 \frac{\mathrm{w}}{\mathrm{m}^{2}}
\end{aligned}
$$

SAM FIN EFFICIENCY $=1.0$

$$
Q_{\substack{\text { Tate } \\ \text { Var }}}=q^{\prime \prime} A=1940 \frac{\mathrm{w}}{\mathrm{m}^{2}}\left(5.48 \mathrm{~m}^{2}\right)=10,631 \mathrm{~W}
$$

FLU WILL BE LIMITED BY AMOUNT OF HEAT VAPORIZE. CAN PRUDE RATHER THAN FRICTION PRESSURE DROP. THCZFFORE A BETTER CALCULATION JP THE VAPORIzER CAPACITY is PRUDEN, 
PRESS. BLDG. cur EAIUUE CASE 3

$10 / 24 / 96$

Raw calls.

RUSS RUCiNski

A

- vaporizer heat transfer capacity.

Assume heat transfer rate inside of TUBES IS $\rightarrow$ OUTSIDE SINCE NUCLEATE BOILING INSIDE \& FREE CONVECTION OUTSIDE.

$$
\begin{aligned}
& T_{\text {OUTS IDE }}^{T_{\text {TUES }}}=T_{\substack{\text { SJ } \\
\text { 102 PSIa }}}=99 \mathrm{~K} \\
& T_{\text {AMBiENT }}=300 \mathrm{~K} \quad\left(80^{\circ} \mathrm{F}-\right.\text { a summer am) }
\end{aligned}
$$

The heat transfer model that describes this SITUATION IS FREE CONVECTION WITHIN ISOTHERMAL parallel plate channels.; assume trans = Tíblis

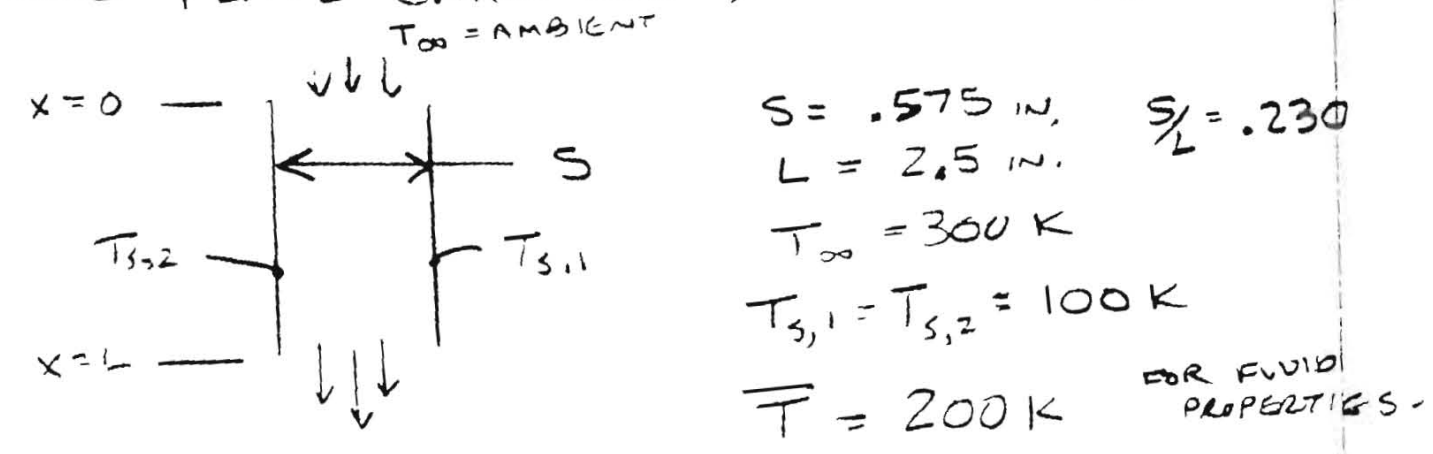

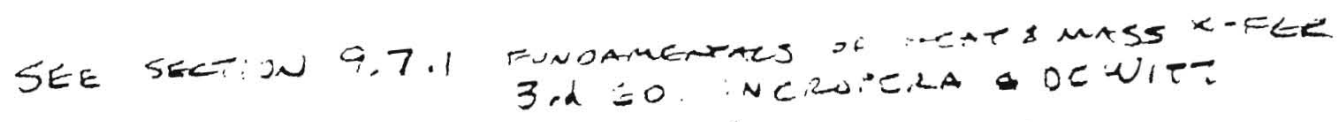

$$
\begin{aligned}
& \bar{N}_{u_{s}}=\frac{1}{24} \operatorname{Ras}\left(\frac{s}{L}\right)\left\{1-\exp \left[-\frac{35}{\operatorname{Ras}_{s}(S / L)}\right]\right\}^{3 / 4} \operatorname{EaN} .
\end{aligned}
$$

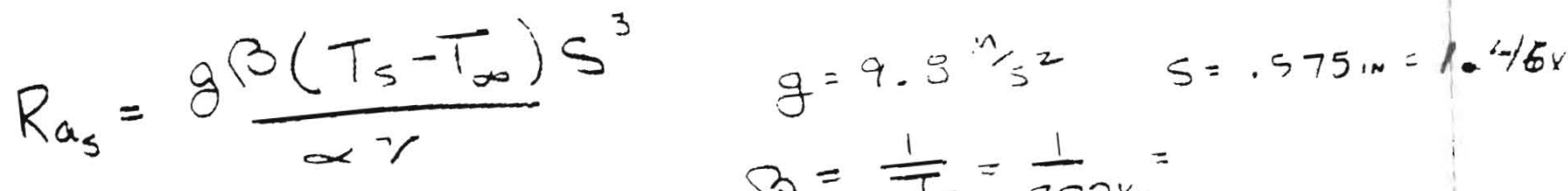

$$
\begin{aligned}
& \beta=\frac{1}{T_{I}}=\frac{1}{200 k}=
\end{aligned}
$$

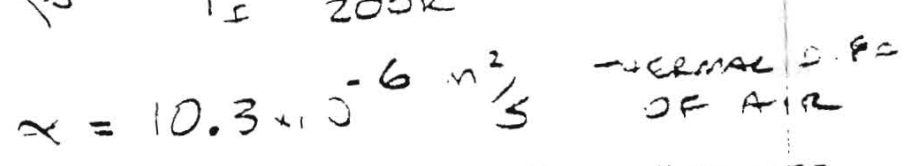

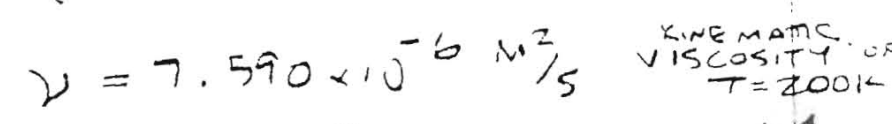

$$
\begin{aligned}
& R_{x_{3}}=\frac{(9.5 \mathrm{~m} / \mathrm{s})\left(\frac{1}{200 \mathrm{~K}}\right)(100 \mathrm{~K}-300 \mathrm{k})\left(1.46 \times 10^{-2} \mathrm{~m}\right)^{3}}{\left(10.3 \times 10^{-6} \frac{\mathrm{m}^{2}}{\mathrm{~s}}\right)\left(7.59 \times 10^{-6} \frac{\mathrm{m}^{2}}{\mathrm{~s}}\right)} \\
& \text { tabere A.4 } \\
& R_{a c}=-3.9053 \times 10^{5} \text { Laminar free convex.... }
\end{aligned}
$$




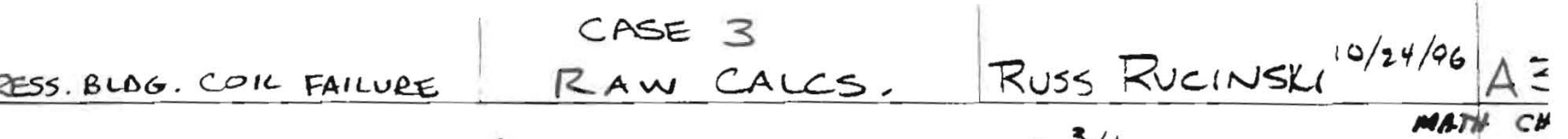

$$
\begin{aligned}
& \sqrt{u_{s}}=\frac{1}{24} \operatorname{Ras}(s)\left\{1-\exp \left[\frac{-35}{R a_{s}(s / L)}\right]\right\}^{3 / 4} \\
& N_{u_{s}}=\frac{1}{24}\left(43.9053 \times 10^{5}\right)(-23)\left\{1-\exp \left[\frac{-35}{\left(+3.9053 \times 10^{5}\right)(-23)}\right]\right\}^{3 / 4} \\
& \bar{N}_{u_{s}}=\quad\left\{3.89584 \times 10^{-4}\right\}^{3 / 4} \\
& N_{u_{s}}=10.378 \\
& \text { * } \bar{N} u_{s}=\left(\frac{G / A}{T_{s}-T_{\infty}}\right) \frac{S}{K} \quad \operatorname{EON} 9.37 \\
& K_{\substack{\text { Ali } \\
200 \mathrm{~K}}}=18.1 \times 10^{-3} \mathrm{~W} / \mathrm{mK} \text { tabLE A.4 } \\
& \text { Re-Anatering } 9.37 \\
& g_{A}=\frac{N_{s}}{s} k\left(T_{S}-T_{\infty}\right)=\frac{(10.378)\left(18.1 \times 10^{-3} \frac{\mathrm{W}}{\mathrm{nk}}\right)}{\left(1.46 \times 10^{-2} \mathrm{~m}\right)} \\
& q / A=-2573 \quad \frac{W}{m^{2}}
\end{aligned}
$$

STILL WE- FIN EFFICIENCY $=1,0$ TO $3 E$ CONSERUNTIC THIS WILL MORE THEN COMPENSATE FOR NOT INGLUDI G THE SURF AGE AREA UF THE bARE TJBES.

$$
\begin{aligned}
& Q_{\text {aAA }}=8 / A \cdot A_{f, M s} \neq 2573 \frac{w}{m}=\left(5.48 \mathrm{~m}^{2}\right) \\
& Q_{\text {Tor }}=14,100 \text { Watts THK } * \text { is }
\end{aligned}
$$

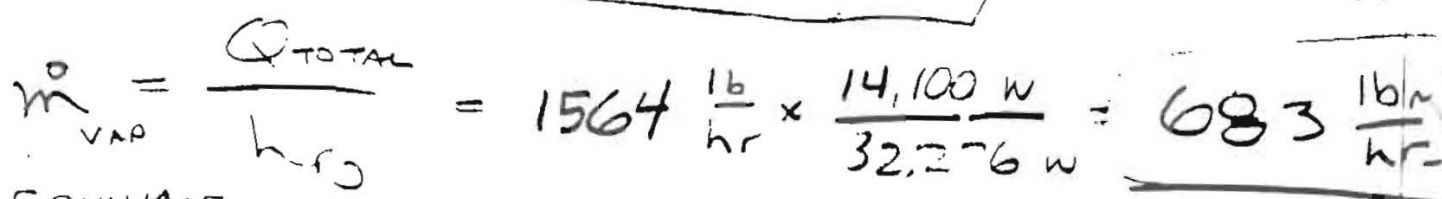

AIr EQUIVALENT:

$$
Q_{a}=\frac{3.1}{60} T \frac{356}{C} \sqrt{\frac{Z T}{M} \frac{28.97}{520}}
$$

EQN. $6 A-1$ TECH. OF LH E 


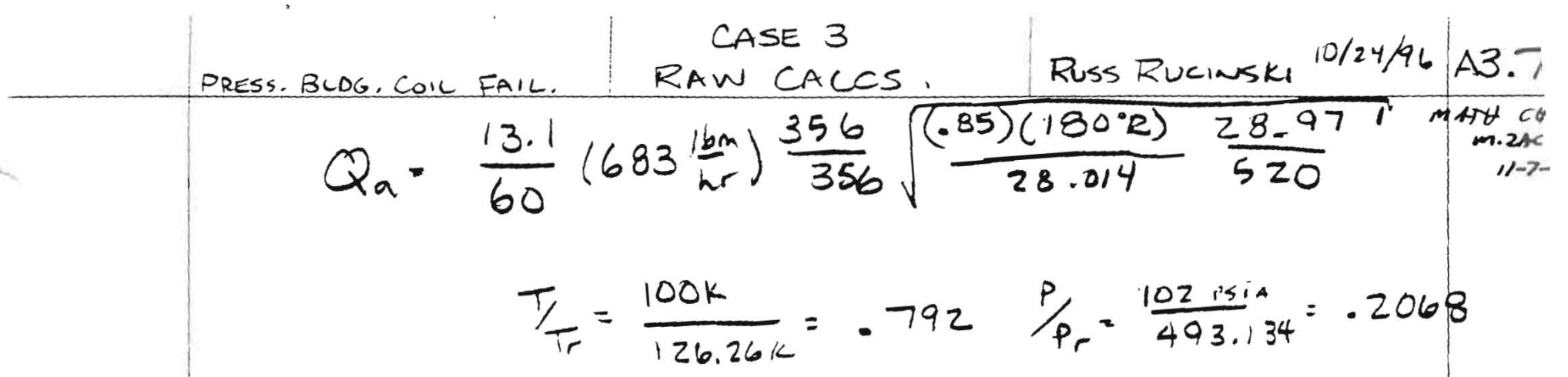

$Z=0.85$ frem Fig. A.5 GeNERAL compeess: Fundanentous of classicmiting. 3'ED. Van WTLEN SOAN

$$
Q_{a}=82.3 \text { scFn AIR }
$$




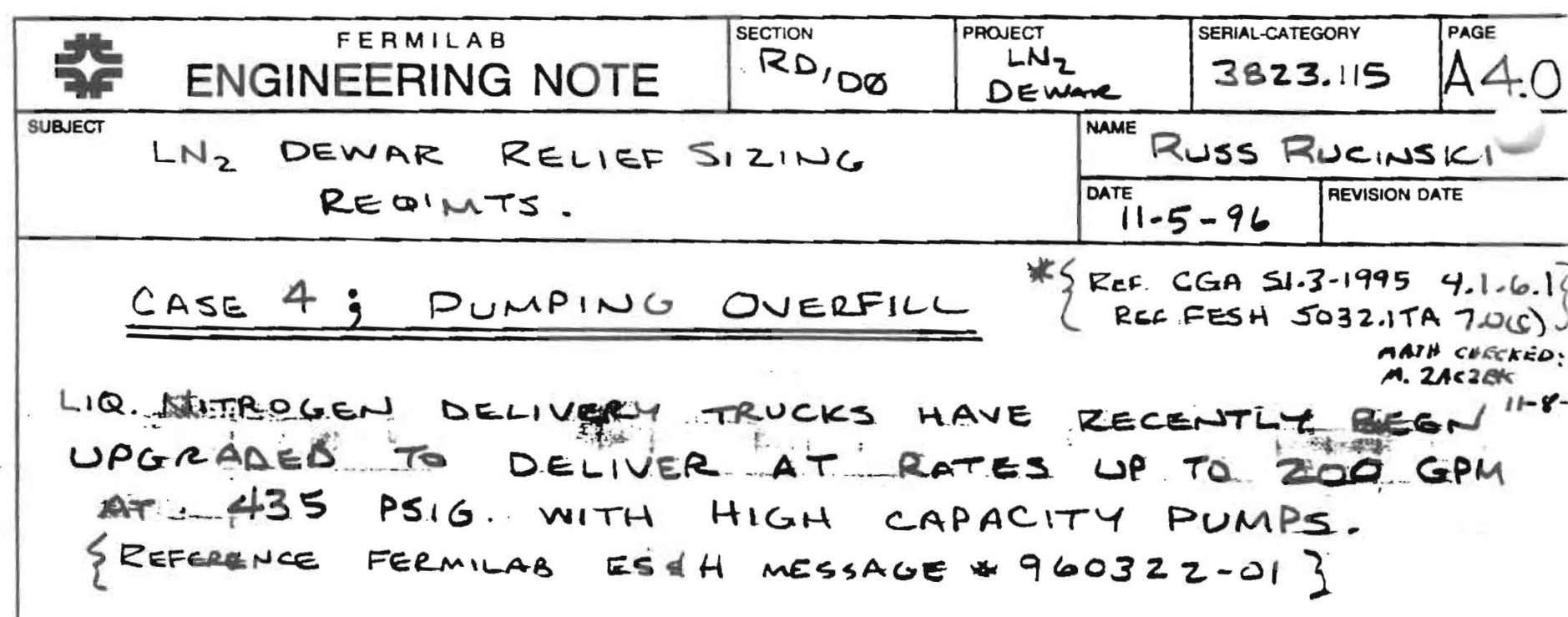

The relieving requirement for such a large Flow rate canNoT be met BU CONUENTIONAL means. therefore, a suitable alternative METHOD has beEN CHOSEN.

Vessel overpressure due to FILLING is PREVENTED BM AN AUTOMATIC SHUT OFF valve installed on the fill line. A SIMPLIFIED SYSTEM SCHEMATIC IS SHOWN BELOW:

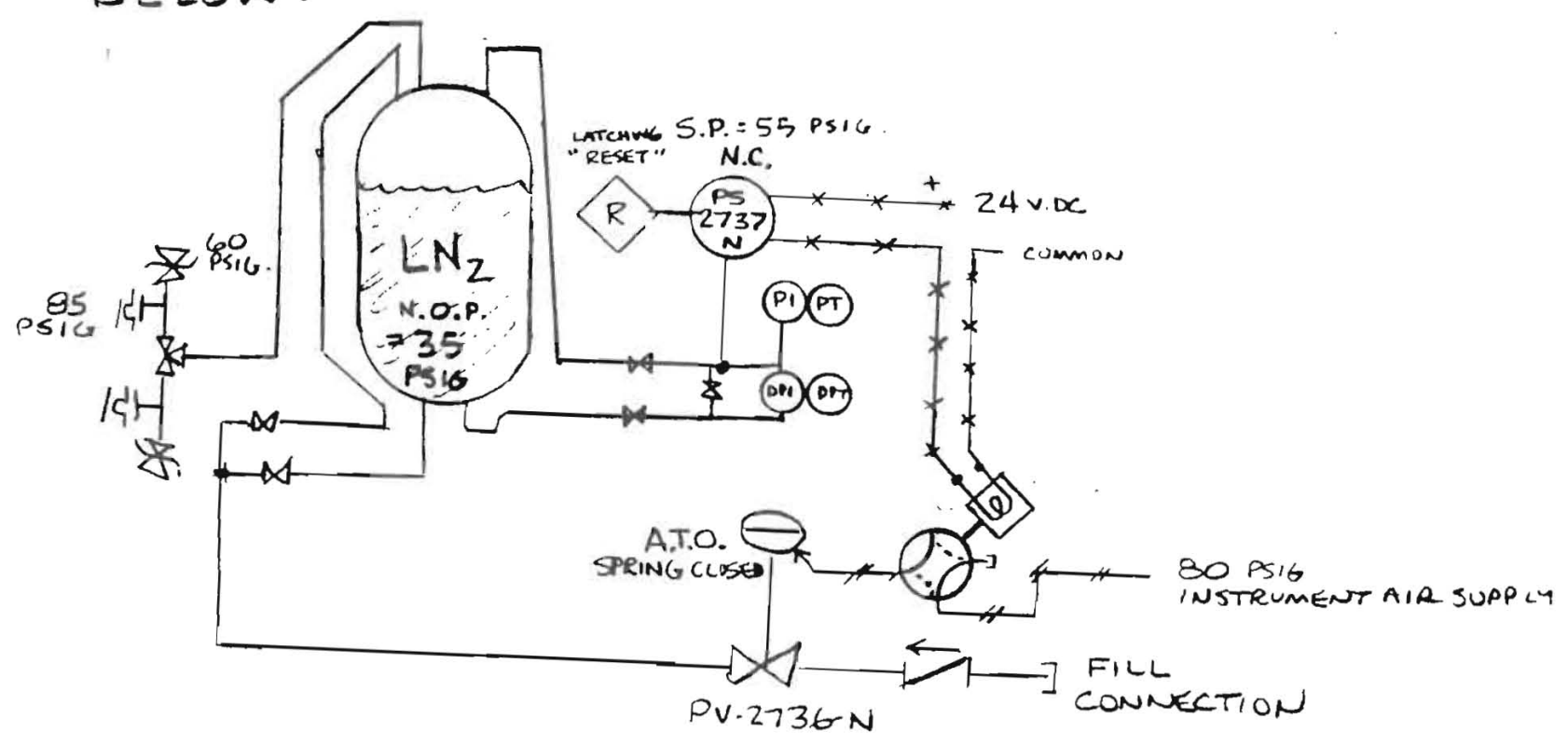

SKETCH 4.1 SIMPLIFIED SCHEMATIC of automatic Fill SHUT-OFF 


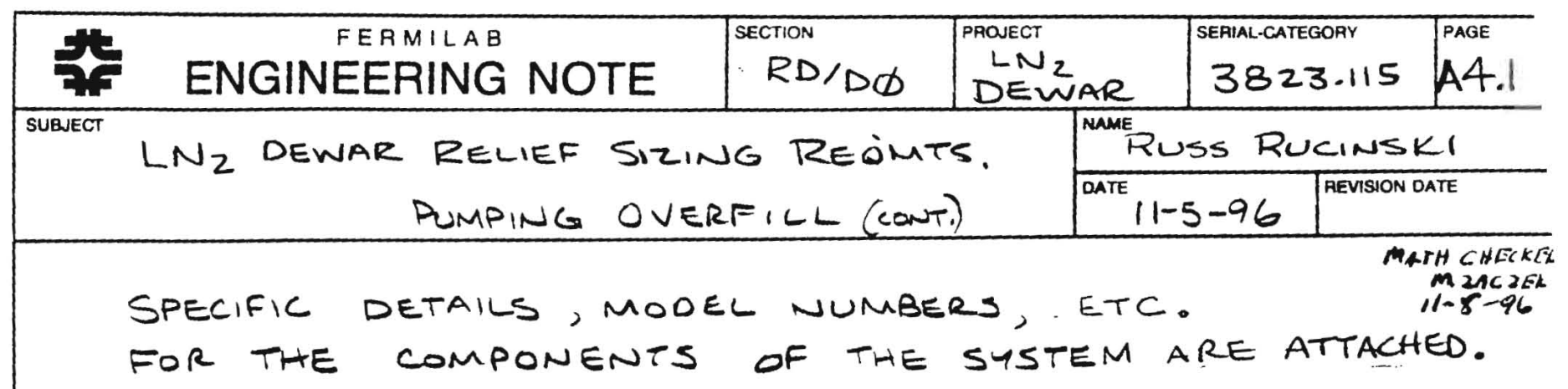

THE NORMAL operating PRESS URE OF THIS DEW TAR IS 35 PIG. DURING A NORMAL FILL THIS PRESSURE SHOULD NOT VARY MORE THAN 5 PSI. FOR A FILLING ACCIDENT case (ériver disRegards level gage.? the PRESSURE SWITCH, PS 2737-N WILL TRIP AT A FIXED SET POINT OF 55 PSI. WHEN TRIPPED, IT BREAKS THE CIRCUIT SUPPLYING POWER TO THE 4 WAY SOLENOID. THE 4 WAY SOLENOID blocks aND VENTS THE AIR SUPPLY THAT HOLDS OPEN THE FILL Valve pV.2736-n. The fill VAlVe closes WHICH PROTECTS THE VESSEL FROM THE SOURCE OF OVERPRESSURE.

THE PRESSURE SWITCH IS PROVIDED WITH A LATCHING RESET BUTTON SO THAT MANUAL INTERVENTION is NECESSARH TO RESUME FILLING OPERATIONS ALL COMPONENTS ARE COMMERCIALLY AVAILABLE, COMMON ITEMS WITH GOOD RELIABILITY. THE SYSTEM IS FAIL SAFE IN AS MUCH AS A LOSS of EITHER ELECTRICAL POWER OR INSTRUMENT air will close the fill valve pV-2736-N.

a reliability analysis follows and stows THat THE SMSTEM is AS SAFE as a class $\phi$ OOH AREA. 


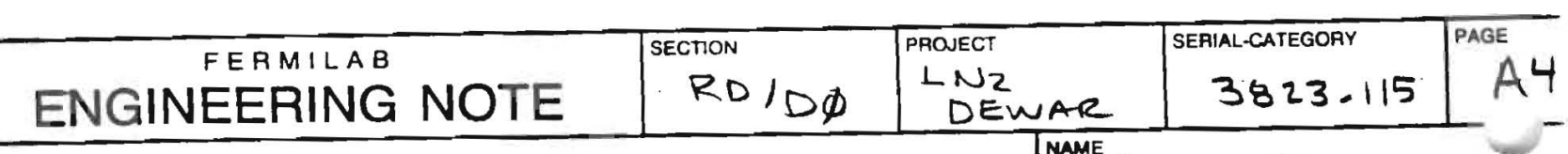

SUBJECT

LN z Dewar ReLIEF SIZING REQIMTS PUMPING OVERFILL (CONE.) Russ Rucinsk 1 $11-7-96$

Reliability/ Probability analysis

FOR AUTO FILL SHUT - OFF ON OJERPRESSU

I WILl USE A PROBABILITM TREe DIAGRAM THERP MODEL \{REF. "HUMAAN FACTURS" BY KantonItZ SORKIN, ISBN 0-471-09594-X\}

Filling event

MAN CHE 14.21628
$11-8-96$

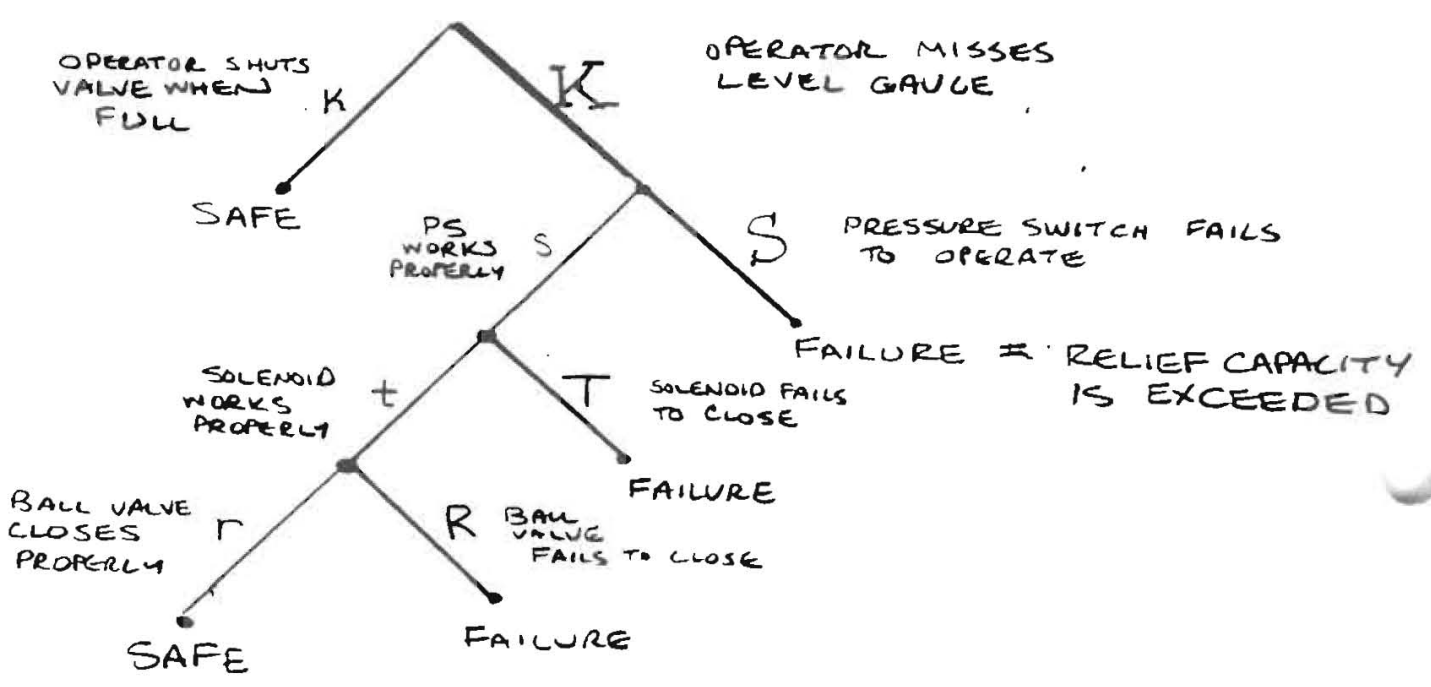

Probabilities

$$
\begin{aligned}
& K=.01 \quad K=99 \quad \text {.On PROBABiLITY OF FAILURE TO RECOCNIZ } \\
& R=.0003
\end{aligned}
$$

ProbabiLITY OF = Pr

$\operatorname{Pr}\left(\begin{array}{c}\text { SAFE } \\ \text { OUTCOME }\end{array}\right)=K+K(S \mid K)(t \mid(S \mid K))(r|t|(S \mid K))$

$$
\operatorname{Pr}\left(\begin{array}{l}
\text { SAFE } \\
\text { OUTCOME }
\end{array}\right)=.99+.01(.99999)(.999)(.9997)
$$

$99 \%$ PROB. OPEOANTIN Givers WFF FlOW

13. proansiuty Pros. P.S.

ITERATOR

OPERATES

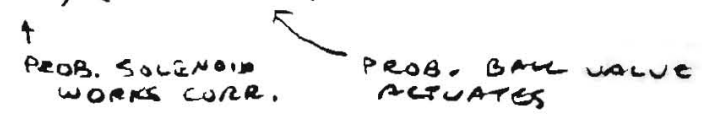




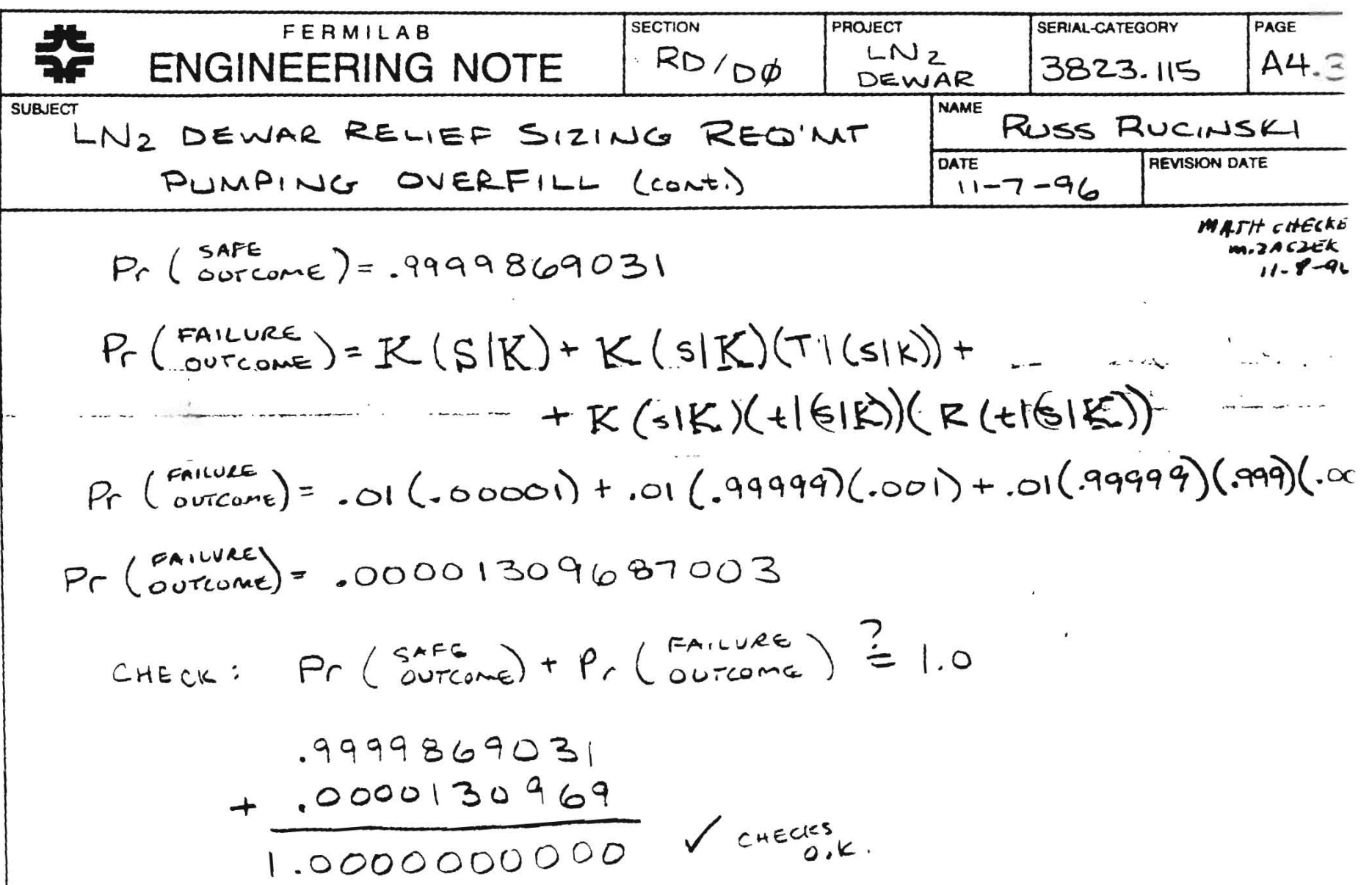

What this means in more tangible terms is,

$$
\left[\operatorname{Pr}\left(\begin{array}{c}
\text { Failure } \\
\text { outcome }
\end{array}\right)\right]^{-1}=76,354
$$

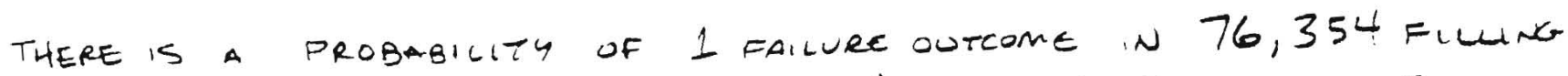
OPERATIONS. ASSUMING 3 FILLS/ WEEK; THIS MEATS 1 faIlure in 489 yeArs. PutTing THIS IN ODH PROBABILITY UNITS OF $\mathrm{hr}^{-1}$;

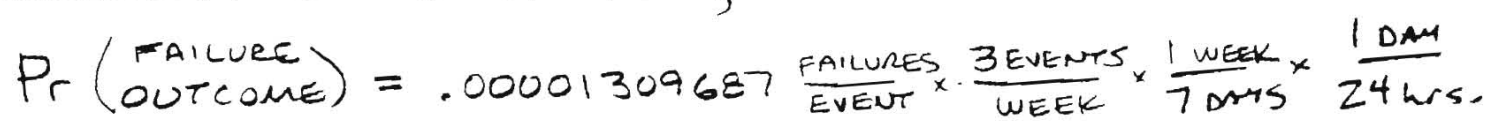

$$
\begin{aligned}
& =2.34 \times 10^{-7} \frac{\text { FAiLURES }}{H r}
\end{aligned}
$$

A failure outcome just means the relief capacity WOULD bE EXCEEDED IF THE PUMPING RATE WAS GREATER HAN $110 \mathrm{gPM}$ (FROM CAPACITY CALLS. NOT presented.) DURING tHAT FILL EVENT. JUST FOr DISCUSSION, ASSUME a one in ten fatality factor fur a FAILURE EUENT. THEN the fatality rate wOULD BE:

$$
\phi=\operatorname{Pr}_{i} F_{i}=\left(2.34 \times 10^{-7} \frac{\text { manures }}{\mathrm{hr}}\right)\left(\frac{1 \text { fatality }}{10 \text { failuas }}\right)=2 \times 10^{-8} \mathrm{hr}^{-1}
$$




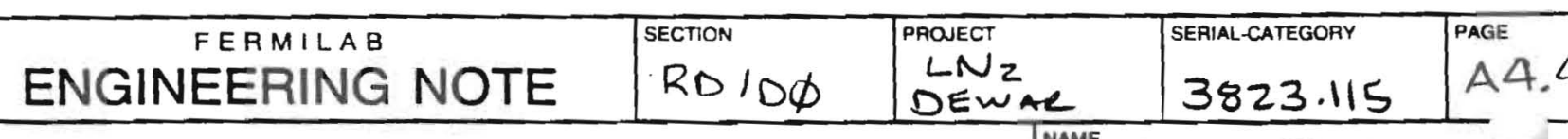

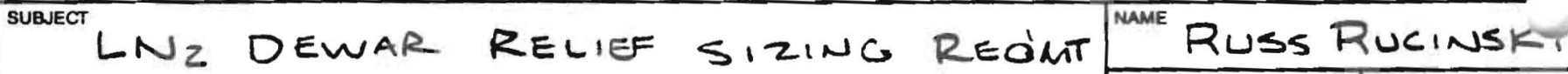
PUMPING OVERFILL (CONT.)

DARE $11-7-96$

REVISION DATE

MATH CHECK

FOR COMPARISON, THE DH CLASS CRITERiA IS THAT IHS $\phi<10^{-7} \mathrm{hr}^{-1}$ for an ooh class $\phi$ area.

I. CONCLUDE THAT IN GOOD ENGINEERING JUDGEMENT, TH AUTO FILL SHUT OFFSUSTEM ON OVERPRESSURE is reliable enOUgh to reduce the hazard to an acceptable level.

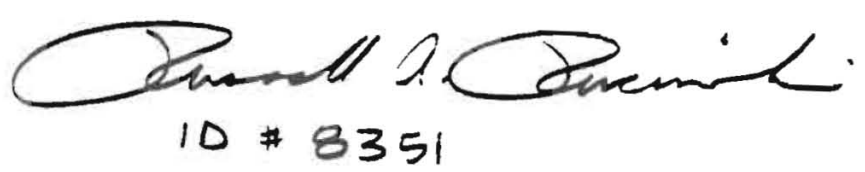


Russ Rucinski EMPLOYEE NO TEXT (SEE INSTRUCTIONS ON REVERSE TMAL STATION

DESIRED DEUVERY
REQUESTORS IDENTIFIER

$12 / 9 / 96$

SUGGESTED VENDOR

RD/DO

PAOJECT DESCRIPTION

357

B\&B Instruments, Inc.

219-932-4474

DØ Upgrade Solenoid

6444 Columbia Ave., PO box 4039

Hammond, IN 46324

DELIVER TO (INCLUDE NAME \& BUILDING NO)

Russ Rucinski, D-Zero Ass'y Bldg.

\section{SHORT DESCRIPTION (30 CHARACTERS OR LESS) \\ Pressure switches - LN2 dewar}

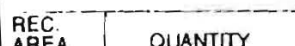

- LN2 dewar

REC
AREA
COD

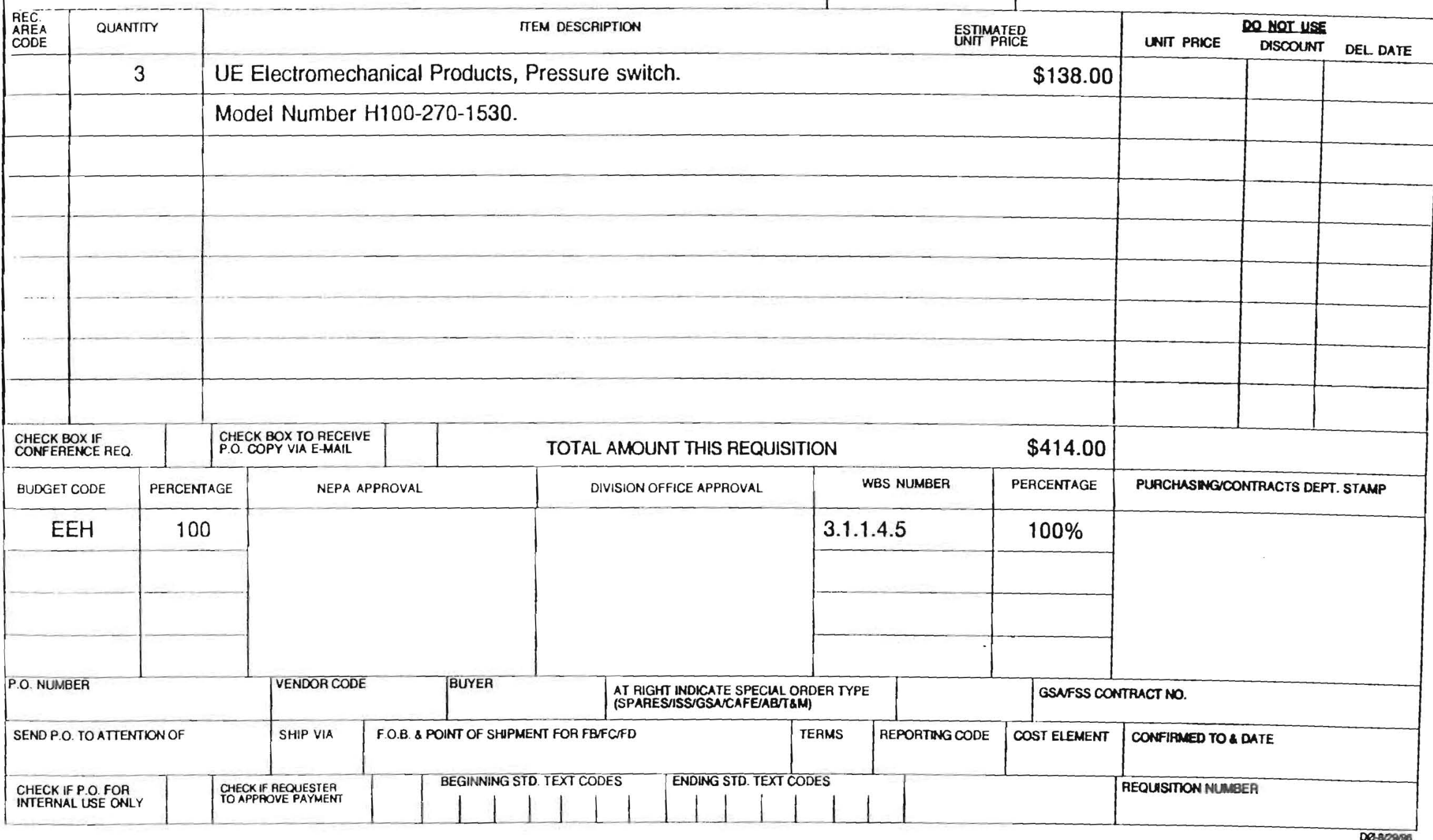

TEM DESCRIPTION

APPROVALS

$11 / 5 / 96$ 


\section{블}

(7)

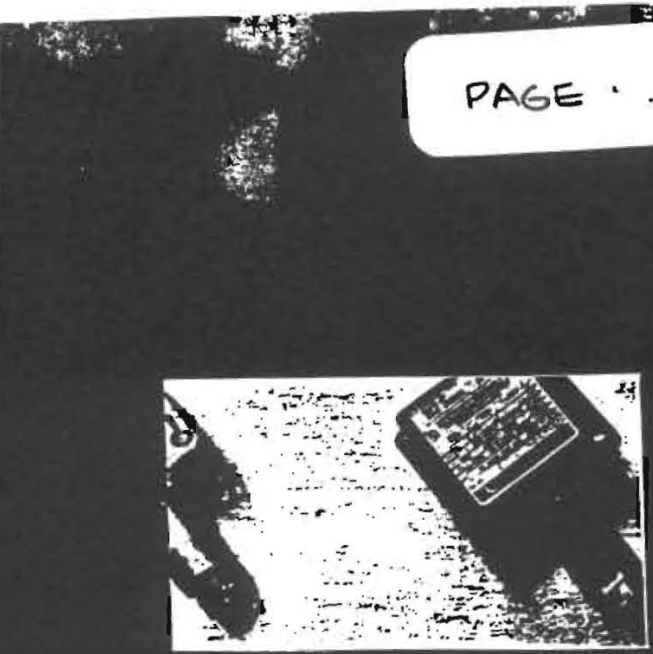

UEElectromechanical Products

Pressure and Temperature Switch Indicating Temperature Contrors Temperature Recorders

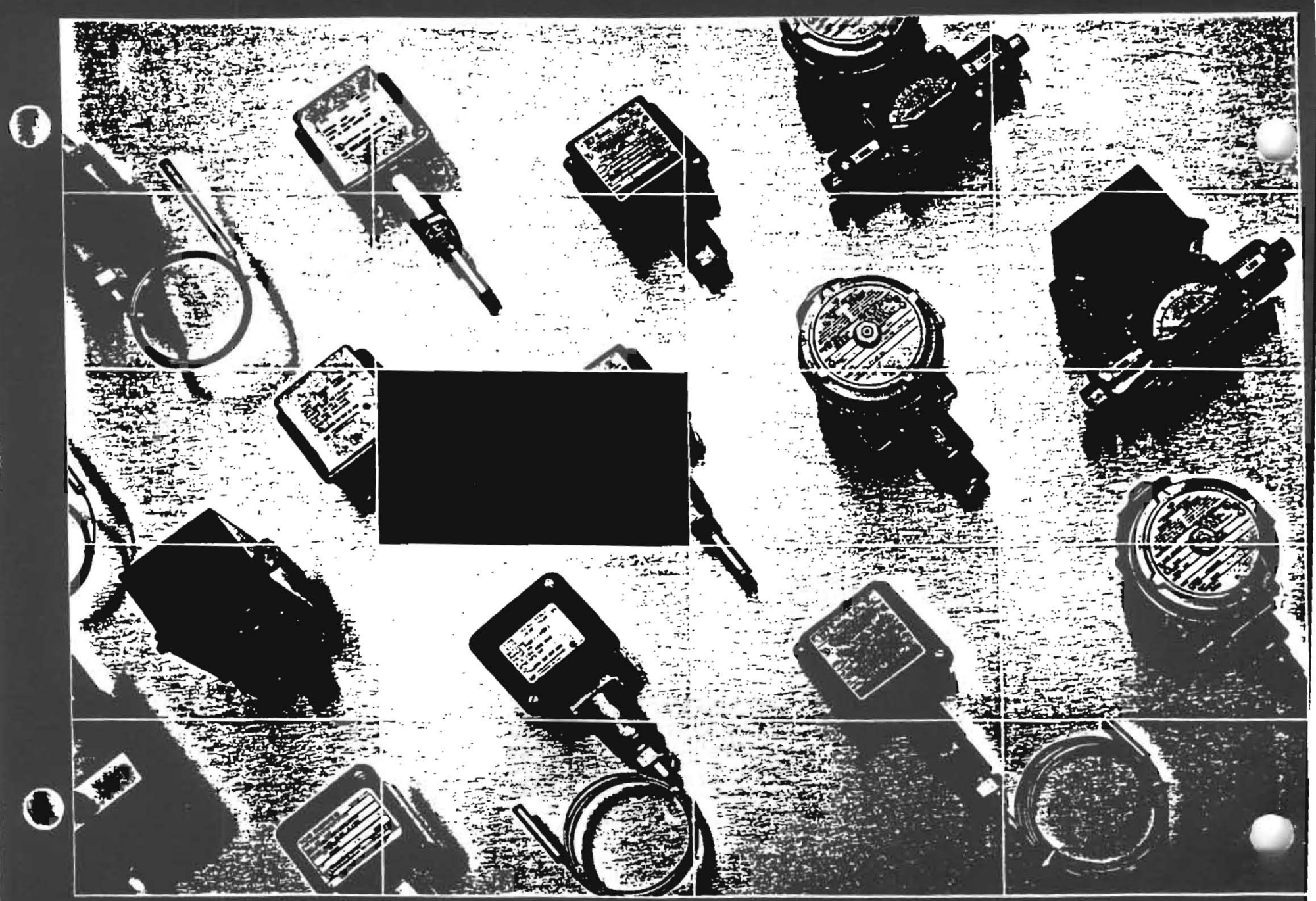



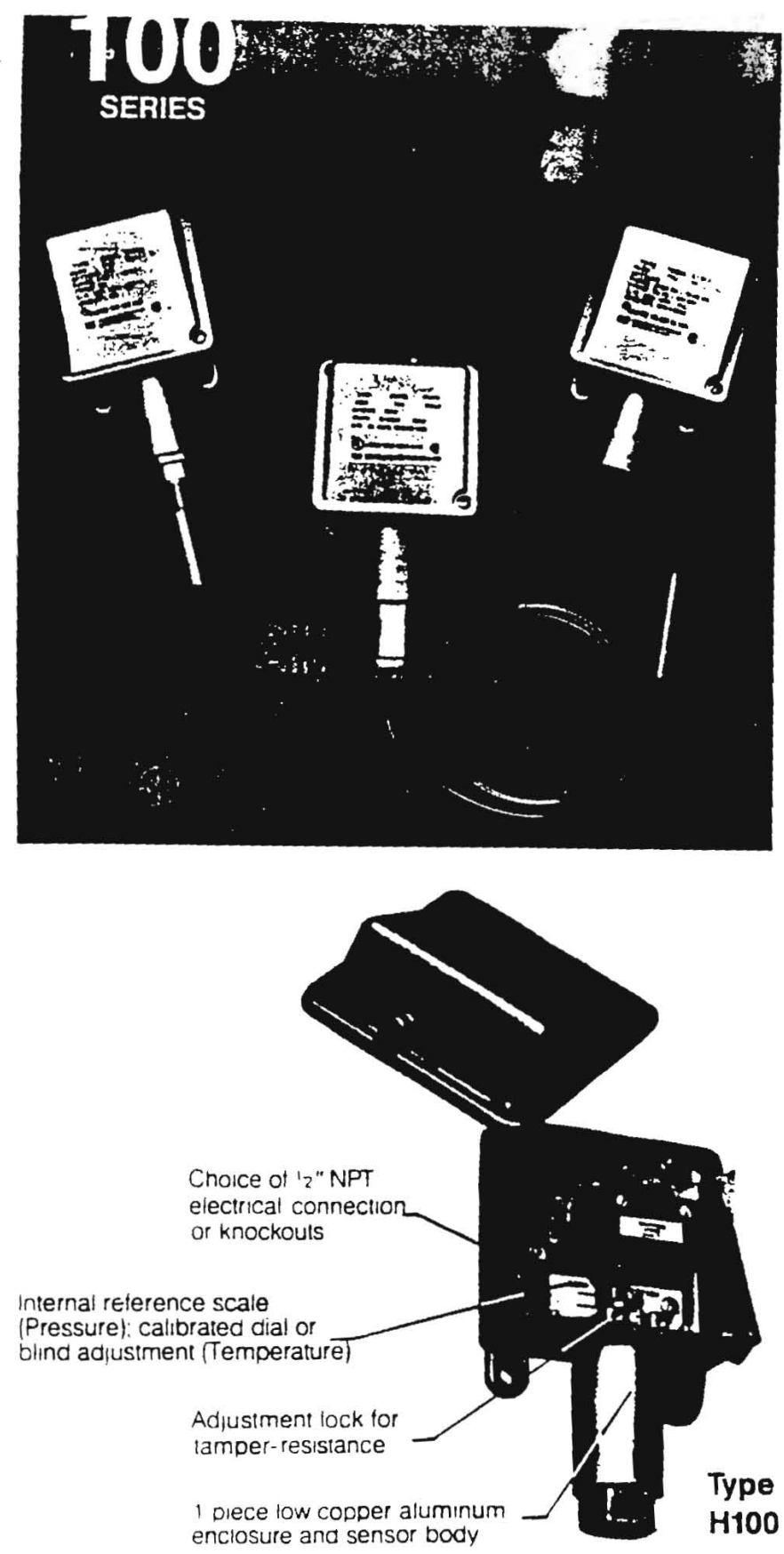

\section{SWr:TEE Tor IVEIVIA 4, Generai Purpose Service

- Introducing a family of low-cost pressure and temperature switches for the process plant or OEM

- Wide selection of adjustable ranges within 3-5000 psi and -180 to $1000^{\circ} \mathrm{F}$

- Internal reference scales and adjustment lock

- Enclosure design permits safe and easy access for wiring

- Many models available from stock

- Factory setting

\section{Specifications}

Approvals:

Storage Temperature:

Ambient Temperature limits:

Shock:

Vibration:

Enclosure Classification:

Set point Repeatability:

Switch Output:

Electrical Rating: Enclosure:

Weight:

Electrical connection: Pressure connection: Temperature Assembly Bulb and Capillary:

Immersion Stem Fill:

Temperature Deadband:

HTFP Heat Tracing/ Freeze Protection:
Ul listed

CSA certified.

$-6510 \pm 160^{\circ} \mathrm{F}$

-40 to $\pm 160^{\circ} \mathrm{F}$, except models $701-706.0$ to $160^{\circ} \mathrm{F}$ Sel point typically shitts less than $1 \%$ of range for a $50^{\circ} \mathrm{F}\left(28^{\circ} \mathrm{C}\right)$ ambient temperature change.

Set pount repeats after $15 \mathrm{G}, 10$ millisecond duration

Set point repeats atter $2.5 \mathrm{G}, 5-500$ CPS.

NEMA 4: (4X see 117 series).

Temperature Models $\pm 1 \%$ of adfustabte range.

Pressure Models 701-706, 270-376 $\pm 1 \%$ of adjustable range. $190-194,612-680 \pm 1.5 \%$ of adjustable range.

1 SPDT. Switch may be wired "normaily open" or "normally closed".

15 amp 125/250/480 VAC resistive.

Die cast aluminum, (max $0.6 \%$ copper)

Light grey aluminum tacquer finish, gasketed

Approx. 2 lbs.

1/2"NPT. (2) 陀" diameter knockouts.

4 "NPTF except models $190-194$ (1/2"NPTF)

6 feet copper or 304 stainless steel models M9B. M9BB 347 stainless steel

\section{Brass}

Models 1BC. 18S are solvent filled models

2.8 are orl-filled and modeis M9B, M9BB are mercury filled.

Typically $1 \%$ of range under laboratory conditions $\left(70^{\circ} \mathrm{F}\right.$ ambient circulating bath at rate of $: 2^{\circ} \mathrm{F}$ per minute change).

Switches designed specifically for heat tracing and treeze protection applications Avallable with types 8100 and E100. Spec fications are the same as above except: B100-13546 includes: 22 amo switch with a stainless steel immersion stem.

E100-13545 includes: 22 amp switch and 10 teet of stainless steel capillary.

\section{Dimensions}

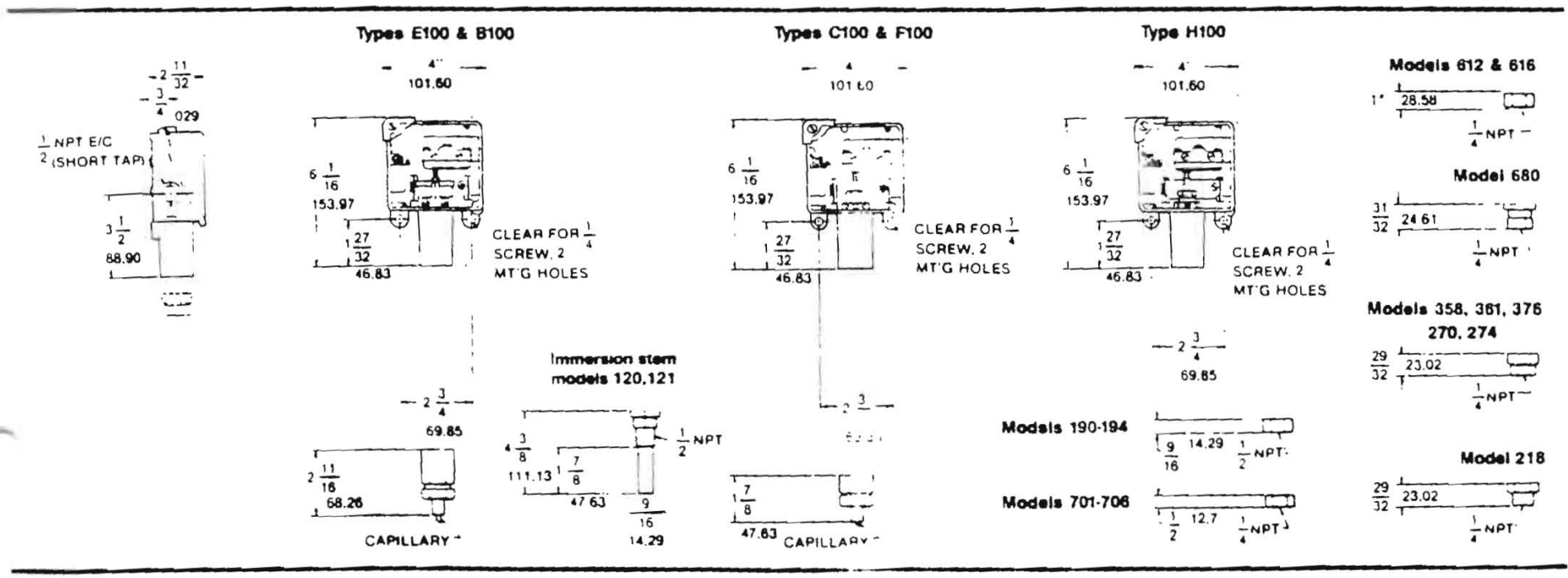




\section{How To Order}

Follow Steps 1.4 to specify the 100

Series pressure and temperature

switch for your application.

1 Specify Type

Pressure:

Type H100 - One SPDT output

Temperature:

Type C100 - Immersion stem,

Type B100 - immersion stem. internal adjustment va calibrated dial

Type F100 - Bulb and capillary. internal adjustment

Type E100 - Bulb and capillary,

$\begin{array}{ll}\text { Bulb and capillary, } & 3 \text { For models not in stock, specify } \\ \text { internal adjustment via } & \text { type and model (eg. H100 mode }\end{array}$ calibrated dial

2 Select standard models in stock for fast delivery. Order by type and stock number (eg. $\mathrm{H} 10 \mathrm{O}$ -
95122). Be sure to check for stocked models with options. If your selection is not stock, go to step 3. 706)

4 If an option is required. add code number from pages 46 and 47 to

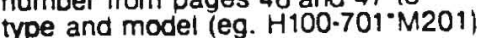

\section{PRESSURE}

\section{Model Chart}

Type H100

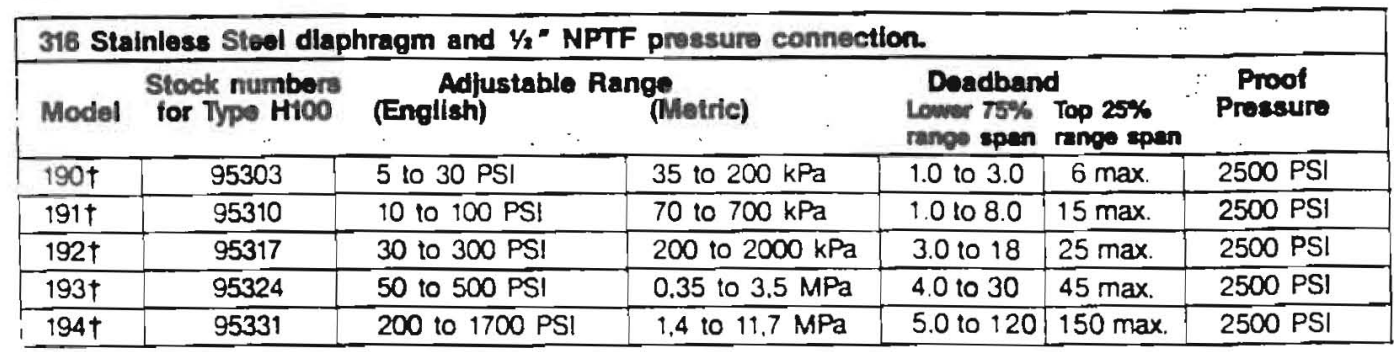

tDeadband Note: Models 190-194 are expressed as the lower $75 \%$ and top $25 \%$ of the range span because of the operating characteristics of the diapnragm sensor and switch.

\begin{tabular}{|c|c|c|c|c|c|}
\hline \multicolumn{6}{|c|}{ Phospher brones bollowrs and $1 / 4$ " NPTF brass pressure connection } \\
\hline Model & $\begin{array}{l}\text { Stock numbers } \\
\text { for Type H100 }\end{array}$ & $\begin{array}{l}\text { Adjustable Ran } \\
\text { (English) } \\
\end{array}$ & (Metric) & Deadband & $\begin{array}{l}\text { Proof } \\
\text { Pressure }\end{array}$ \\
\hline 218 & \begin{tabular}{|l|l|}
95704 \\
\end{tabular} & $30^{\circ} \mathrm{Hg}$ VAC to 0 PSI & -100 to $0 \mathrm{kPa}$ & 1 to 2 " $\mathrm{Hg}$ & $30 \mathrm{PSI}$ \\
\hline 270 & 95166 & 20 to $200 \mathrm{PSI}$ & 140 to $1400 \mathrm{kPa}$ & 1 to 4.0 PSI & $250 \mathrm{PSI}$ \\
\hline 274 & & 30 to $300 \mathrm{PSI}$ & 200 to $2000 \mathrm{kPa}$ & 1 to $5.0 \mathrm{PSI}$ & $350 \mathrm{PSI}$ \\
\hline \multicolumn{6}{|c|}{316 Stainless steel bellows and $1 / 4$ " NPTF pressure connection } \\
\hline 358 & & 20 to $200 \mathrm{PSI}$ & 140 to $1400 \mathrm{kPa}$ & 1 to $3.0 \mathrm{PSI}$ & $250 \mathrm{PSI}$ \\
\hline 361 & & 30 to $300 \mathrm{PSI}$ & 200 to $2000 \mathrm{kPa}$ & 1 to 1.4 PSI & 350 PSI \\
\hline 376 & & 50 to $500 \mathrm{PSI}$ & 0.35 to $3.5 \mathrm{MPa}$ & 1.5 to $5.0 \mathrm{PSI}$ & $575 \mathrm{PSI}$ \\
\hline \multicolumn{6}{|c|}{318 Stalnless steol bollows and $1 / 4$ " NPTF pressure connection } \\
\hline 680 & 95415 & 100 to $1700 \mathrm{PSI}$ & 7 to $11.7 \mathrm{MPa}$ & 91023 PSI & $2500 \mathrm{PSI}$ \\
\hline \multicolumn{6}{|c|}{$\begin{array}{l}303 \text { Stainiess steel piston and Buna-N }{ }^{-1} \text { ring } 1 / 4^{*} \text { NPTF pressure connection, (not recommended } \\
\text { for gas service since drying of the "O" ring can allow bleeding of medium into the atmosphere). }\end{array}$} \\
\hline 610 & & 100 to $1000 \mathrm{PSI}$ & 0.7 to $7 \mathrm{MPa}$ & 30 to $150 \mathrm{PSI}$ & $10000 \mathrm{PSI}$ \\
\hline 612 & 95712 & 200 to 3000 PSI & 1,4 to $20.0 \mathrm{MPa}$ & 40 to $250 \mathrm{PSI}$ & $10000 \mathrm{PSI}$ \\
\hline 616 & & 700 to $5000 \mathrm{PSI}$ & $4.81035 .0 \mathrm{MPa}$ & 40 to $375 \mathrm{PSI}$ & 10000 PSI \\
\hline \multicolumn{6}{|c|}{$\begin{array}{l}\text { Buna- } N \text { dlaphragm, "O" ing and } 1 / 4 " \text { NPTF brass pressure connectlon. Buna-N dlaphragm limited } \\
\text { to process temperatures below } 165^{\circ} \mathrm{F} \text {. }\end{array}$} \\
\hline 701 & 95122 & 3 to $30 \mathrm{PSI}$ & 20 to $200 \mathrm{kPa}$ & 1.0 to $2.0 \mathrm{PSI}$ & 600 PSI \\
\hline 702 & 95129 & 10 to $100 \mathrm{PSI}$ & 70 to $700 \mathrm{kPa}$ & 1.0 to $4.0 \mathrm{PSI}$ & $600 \mathrm{PSI}$ \\
\hline 703 & 95136 & 30 to $300 \mathrm{PSI}$ & 200 to $2000 \mathrm{kPa}$ & 1.0 to $5.0 \mathrm{PSI}$ & $600 \mathrm{PSI}$ \\
\hline 704 & 95142 & 50 to $500 \mathrm{PSI}$ & $0.35103 .5 \mathrm{MPa}$ & 2.0 to $8.0 \mathrm{PSI}$ & $2500 \mathrm{PSI}$ \\
\hline 705 & 96149 & 100 to $1000 \mathrm{PSI}$ & 0,7 to $7.0 \mathrm{MPa}$ & 3.0 to $20.0 \mathrm{PSI}$ & 2500 PSI \\
\hline 706 & & 200 to $1700 \mathrm{PSI}$ & 1.4 to $11.7 \mathrm{MPa}$ & 10.0 to $30.0 \mathrm{PSI}$ & $2500 \mathrm{PSI}$ \\
\hline
\end{tabular}

-Stocked items with options. 


\section{Options}

Many UE products with options are available from stock. These are indicated in model charts throughout the catalog by a stock number

beginning with the number "g" $(\mathrm{H} 100-95606$, for example, refers to an

$\mathrm{H} 100$ pressure switch with option M315I. Order these by type and stock number (H100-9536, eg.). Switches listed below are UL listed, CSA approved. All switches are SPDT unless statec otherwise

For products with options that are not in stock, follow 1-3.

1. Specify product type $\mathrm{H} 100$

2. Select model from chart $\mathrm{H} 100-70$

3. Select option from these pages after checking availability listed H100-701"0140"M201.

\section{Optlonal Switches \\ Code Description/avaliablilty \\ 01401 amp 125 VAC res. gold flashed contact (dry circuit). Available: \\ Types J400, J\&H402, J403 (C400, 02, 03) (F400, 02, 03) \\ Types J. C. F, 120: E. B. H122 series $J 6$ and 100 \\ 05005 amp 125/250 VAC res. switch, close deadband. \\ Avallable: Series $400,120,100,6,21 \mathrm{~K}, 54,55$ \\ 1010 DPDT switch, 10 amp 125/250 VAC resistive 120 deadbands wider than standard. Available for certain models \\ J120, J120K: all models F120, E121, H100. F100, C100 \\ 107010 amp 125 VDC switch. Available for the falowing: \\ Types J. C. F 400, all type 402 \\ Types C, F, J 120 \\ Series J6, 100 and 54 \\ Deadbands wider than standard. \\ Hermetic Seal \\ SPDT. 11 amp 125/250 VAC. resistive UL. CSA listed. Avalable C. F. $j 120$ \\ 1190 DPDT, 11 amp Hermetlc Seal. Avallable: 117, 120 Series. \\ 151915 amp adjustable deadband 480 VAC res. max. Available: J120.J120K. C120. F120. C. F. H100 \\ 152015 amp 277 VAC res. max., adjustable deadband. \\ Avallable: Series J6 (126-S160. 218-230) \\ Types J. C. F 400 and J. C. F 402 \\ Types $\mathrm{H} 105, \mathrm{~J} 54$ and $\mathrm{J} 54 \mathrm{~S}$ \\ Series J21K \\ $1521 \quad 15$ amp 277 VAC res. max., $\mathbf{2 . 4 0 \%}$ of span adjustable \\ deadband construction. Avallable: \\ J6 (126-S160B) J400, (126-S164B, 451-454) j400K}

$153015 \mathrm{amp} 125 / 250$ VAC res. external manual reset on increasing pressure and temperature.

Types J. C. F 400: J. C. F 402 Series: J21K, J6

Types C. F. H 100: B. C. E. F. J54

Types C. F. J120.'E55, E55A

200020 amp 125/250 VAC res. switch. Avallable:

Types J C F 400.402.403 Series 120 single switch only.

Series $55,100, J 6$, J21K, J27KB, 800, 820

Type E105. B105

\section{Recorder options}

Code Description/avallablilty

MOO2 60Hz, 115VAC, 7 day chart drive

Avallable: 650 Series

M0O3 $50 \mathrm{~Hz}, 115 \mathrm{VAC}, 7$ day chart drive

MOO $50 \mathrm{~Hz}, 230 \mathrm{VAC}, 7$ day chart drive

M009 60Hz, 230VAC, 24 houp rotation

\section{General Options \\ Code Description/avaliability}

M007 Drlled electrical opening

800 Series, right side.

M201 Sot one switch; specify increasing or decreasing pressure or temperature and set point

M202 Set two switches; specity increasing or decreasing

pressure or temperature and set point.

M203 Sat three switches; specify increasing or decreasing

pressure or temperature and set point.

Available: H122K, H121K, J400K, J402K (models 147-S157B)

M270 Meiric calibrated diala (Celsiua)

Dual reference scales standard for many Series. Available: E54. $E 55,650$ 's only.

M300 NEMAA 4 construction

Available for the 800 Series

Standard for many Series.

J21K, 400 Series only requires option M900 for compliance.

M315 Epoxy Polyamide coating of enclosure, exiernal aluminum parts. Available: Types J, C, F 402 Series: 100 Series: Types C F. J 120 . B. E. H122 only.

M321 Gasketed Lexan window

Available on Types, B. E. H 400 Series.

Cenelec Flameproof enclosure, BS5501, part 5. 1977, IP-66.

BASEEFACENELEC EN 50014/50018: EEx $11 \mathrm{~b}$ T6.

Approval available for the 120 Series only. Prefix model number with ' $Q$ ' eg.

QF120-6BS (EExdlle T6-Consult factory).

M410 CSA option

Avallable for the following:

J6, J40, 54

M411 Compliance to NACE std. MR-01-80, 120, 100; 117 Series pressure modeis 190-193. Consult factory.

M444 Paper ID tag

M446 Stainless steel ID tag \& wire attachment

(attachment will vary depending on product)

M449 Mounting hardware

800 Series

M450 Breather drain

Avallable for cerain 120 Series models only. Consult factory

M540 Viton construction (deadbands may increase slightly)

$H 100, H 100 D$. H117, H117D and 120 Series models $700-713$

wetted parts include Viton diaphragm and "O" ring plus 303 St/SI. 1/4" NPTF pressure connection.

120 Series models $450-454,400$ Series models $448-454$

54 Series models $22-28$ plus $120 \mathrm{~K}, 400 \mathrm{~K}$ and $105 \mathrm{~K}$ models

455-457: wetted parts include Viton diaphragm and "O" ring

plus standard connection material.

All products within models $610-618$ and $50-55$ : "O" ring

(only) is changed to Viton.

M550 Oxygen service clesning. Internal construction may change, consult UE. Avaliable for:

Pressure controls (H60, 100, 105, 120, 400 Series).

Not avallable: J400-614, J402-610

Differential pressure controls (120K, 105K, 400K)

M900 Watertight condult titting. Converts $7 / 8^{\circ}$ hole to $1 / 2^{\circ}$ NPT

Available for the following:

400 Series 800 Series

117 Series 650 Series

M901 Watertight conduit titting. Converts $12^{\circ}$ NPT $103 / 4^{\circ}$ NPT.

(117 Series).

Note. For celsius models, std. 24 hour rotation order odtion M270

For celsius models. 7 day rotation suffix " $C$ " after option code (eg: $\mathrm{M002C)}$

-Use of 1530 or M450 options provide CSA. Class I. Div. 1 group C \& D only 


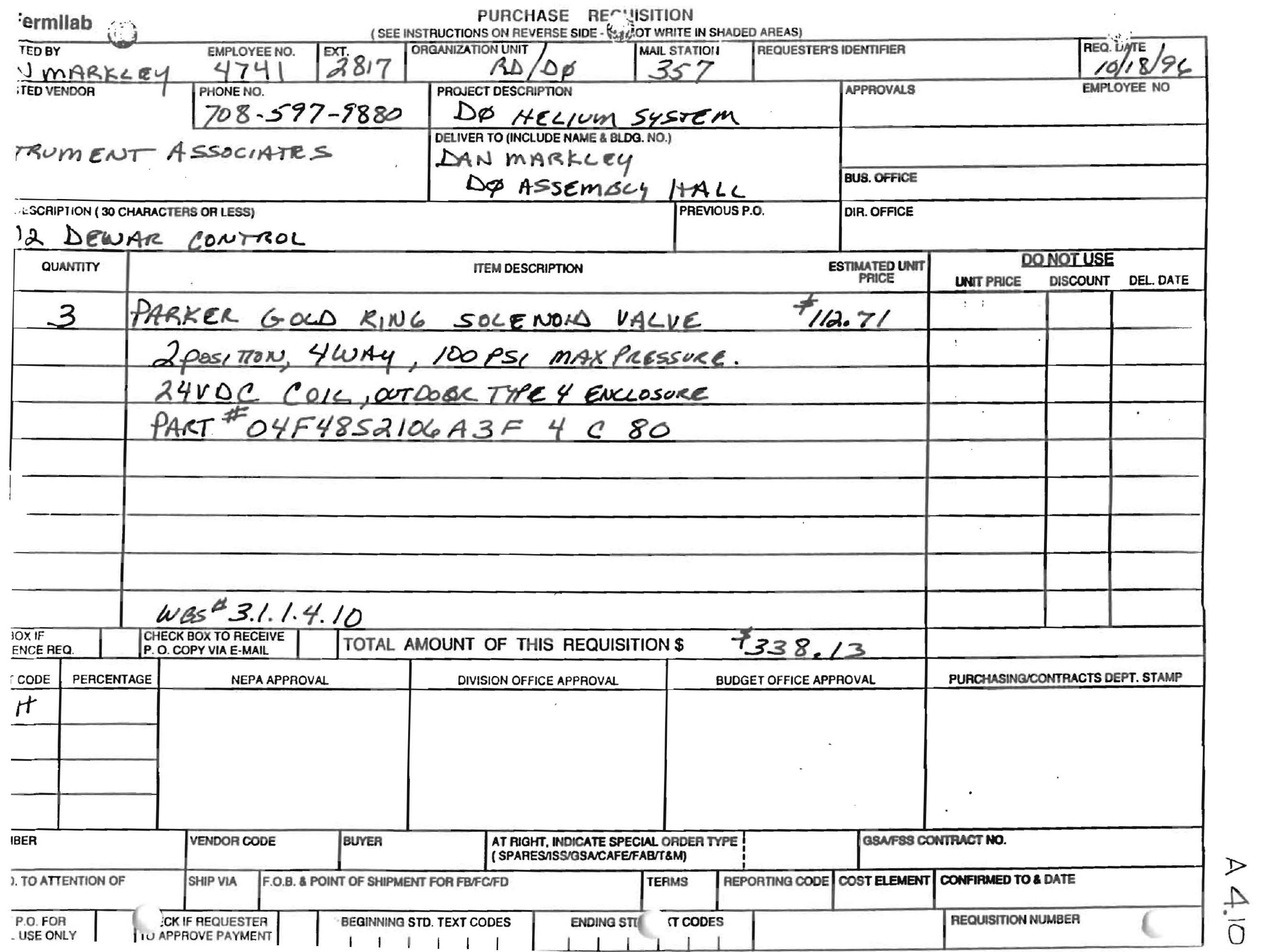




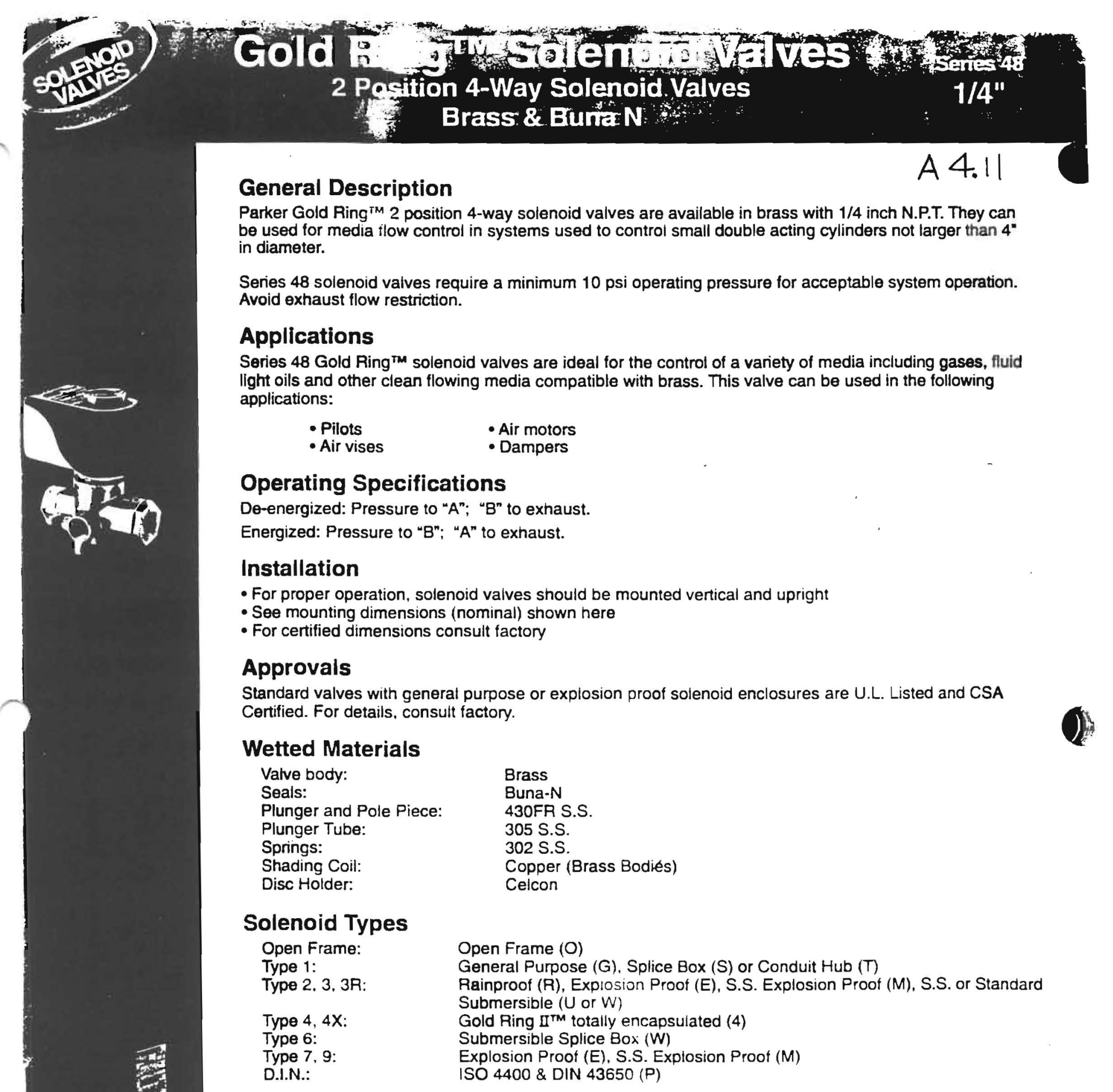

\section{Electrical}

$24 / 60,110 / 120-50 / 60,220 / 240-50 / 60,440 / 480-50 / 60$ volts A.C.

$6,12,24,120,125$ volts D.C. Other voltages as requested.

\section{Coil}

Class F, Standard. Class H Available.

\section{Temperature Ratings}

Media as listed

-A.C. voltages: $180^{\circ} \mathrm{F} \max$ - D.C. voltages: $104^{\circ} \mathrm{F}$ max.

Ambient: $32-77^{\circ} \mathrm{F}$ (standard)

For temperature vanations, consult factory. 


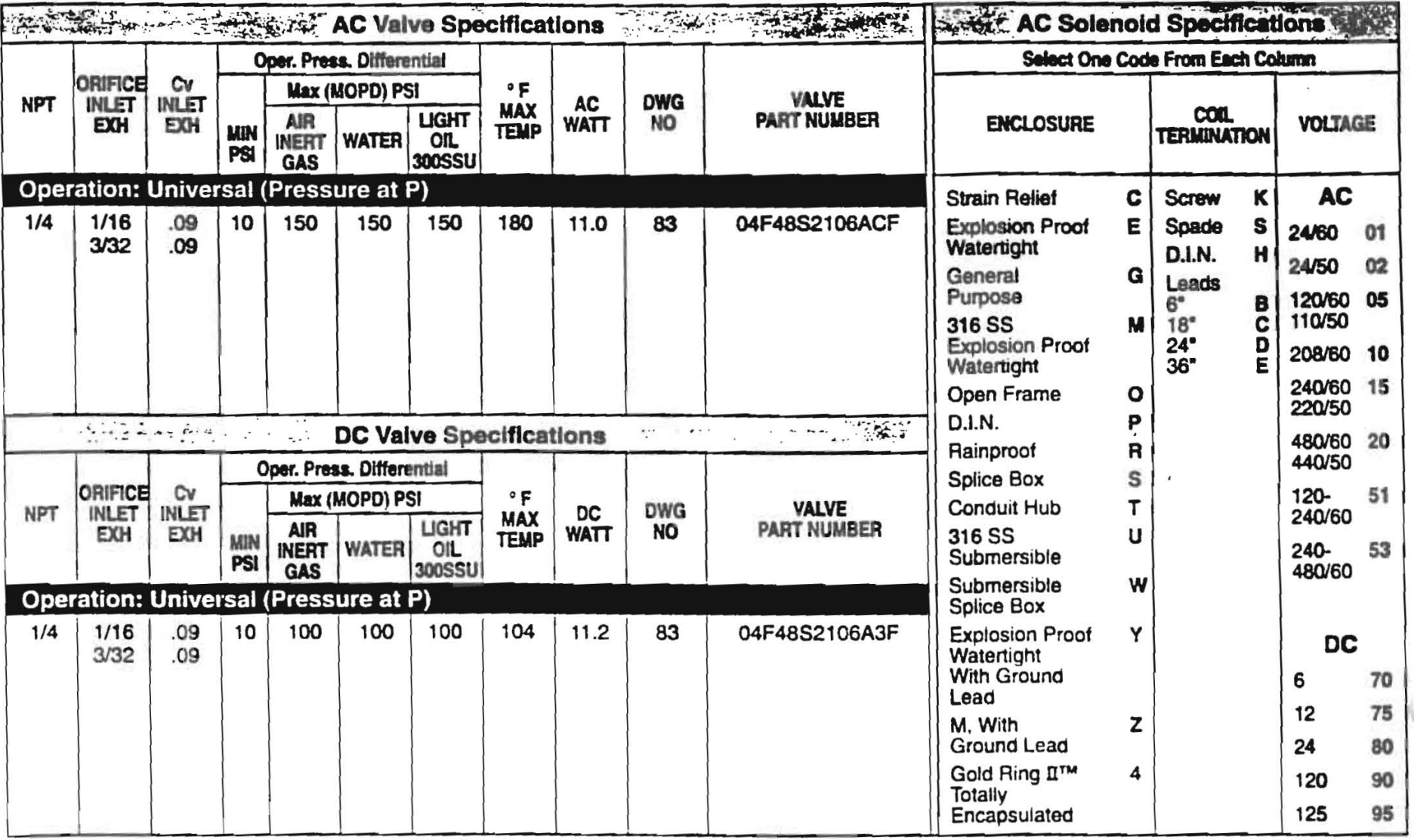
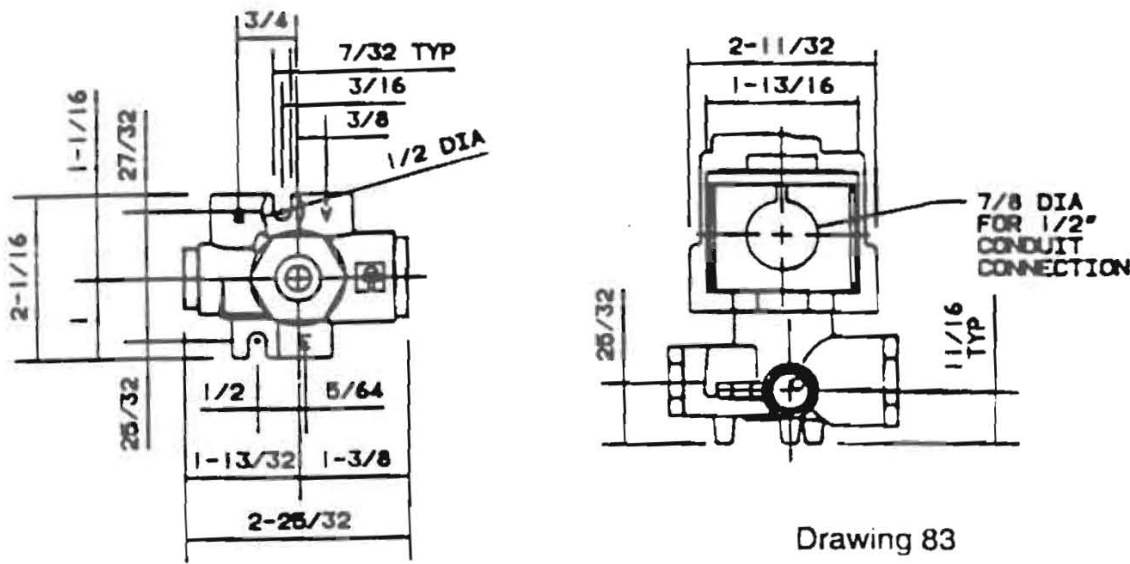

Drawing 83

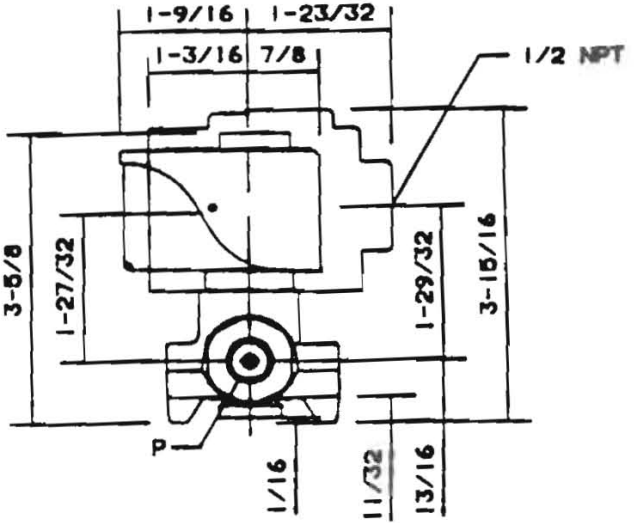

Explosion-Proot/Watertight Shown in Outline 


\section{각 Fermilab}

REQUESTED BY

Russ Rucinski

SUGGESTED VENDOR

Columbia Pipe \& Supply

1803 Moen Avenue

Rockdale, IL 60435
PURCHASE REQUISITION

(SEE WSTRUCIONS ON REVERSE SIDE - DO NOT WRITE IN SHADED AREAS)

\begin{tabular}{|c|c|c|c|c|}
\hline $\begin{array}{l}\text { EMPLOYEE NO EX } \\
8351\end{array}$ & EXT. & ORGANIZATION UNIT & RD/DO & $\begin{array}{c}\text { MAIL STATION } \\
357\end{array}$ \\
\hline
\end{tabular}

PHONE NO

815-729-3200

PROJECT DESCAIPTION

DØ Upgrade Solenoid

DELIVER TO (INCLCLDE NAME \& BULLING NO)

Russ Rucinski, D-Zero Ass'y Bldg.

\section{SHORT DESCRIPTION (30 CHARACTERS OR LESS)}

LN2 dewar valves

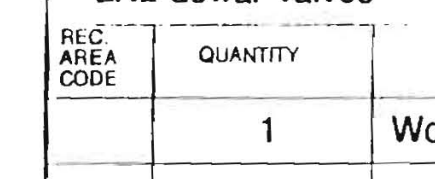

Worcester 1-1/2" C4466-PM-SW-V1 Cryogenic diverter ball valve

with extended stem handle. 300 series stainless steel body, ball \& stem.

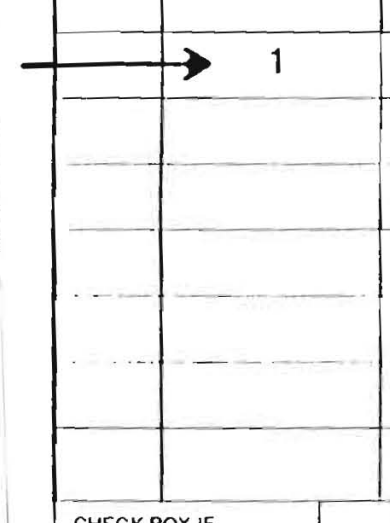

Worcester 1-1/2" C4466-PM-SW Cryogenic C4 Ball valve with mounted

actuator \#2039SN, Spring return, air to open, fail closed, no solenoid.

300 series stainless steel body, ball \& stem. Will be operated

with 80 psi air supply.

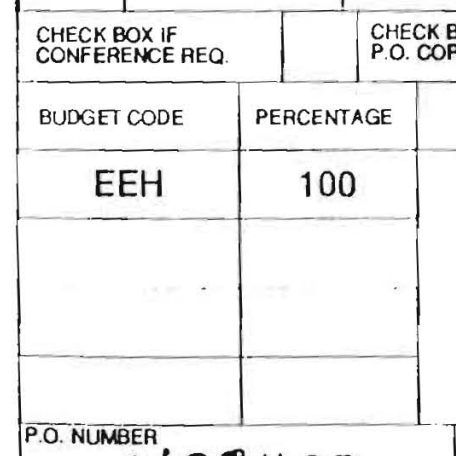

\section{P. NUMBER 98400}

SEND P.O. TOATTENTION OF

VENDOR CODE

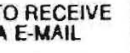

VITAL AMOUNT THIS REQUISITION

\begin{tabular}{|l|l|}
\hline WBS NUMBER & $\$ 2,100.00$ \\
\hline & PERCENTAGE \\
\hline
\end{tabular}

DESIAED DELIVERY

12/10/96

REQUESTOR'S

\begin{tabular}{|c|c|c|}
\hline & DESIAED DELIVERY & $12 / 10 / 96$ \\
\hline IDENTIFIER & & $\begin{array}{l}\text { REQ. DATE } \\
11 / 1 / 96\end{array}$ \\
\hline
\end{tabular}

APPROVALS

EMPLOYEE NO

DIR. OFFICE

$-$

AT RIGHT INDICATE SPECLAL ORDER TYPE

\begin{tabular}{|l|l|}
\hline 3.1 .1 .4 .5 & $100 \%$ \\
\hline
\end{tabular}

PURCHUSINGCONTRACTS DEPT. STAMP

INTERNAL USE ONLY

F.O.B. \& POINT OF SHIPMENT FOR FBFCFD

GSAFSS CONIRACT No.

COST ELEMENT

CONFIPAMED TO \& DATE 


\section{C4 and C4 Diverter Valves with Stem Extensions}

\begin{tabular}{|c|c|}
\hline Vahre Slzes: & 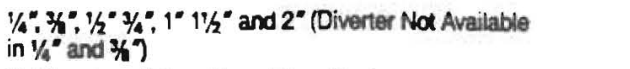 \\
\hline Styles: & $\begin{array}{l}\text { Unidirectional Fow, Threopiece Design } \\
\text { Extended, OnoPiece, Blowour-Proof Stem Diverter-V1, V2 Porti }\end{array}$ \\
\hline Body: & $\begin{array}{l}\text { ASTM A743 CF3M. 316L Stainiess Stoet } \\
\text { ASTM B283 C37T00, Brass }\end{array}$ \\
\hline Plpa Ends: & $\begin{array}{l}\text { ASTM A743 CF3M, (316) Stainless Stoel } \\
\text { ASTM A743 CFBM, (316) Stainless Stoel } \\
\text { ASTM B283 C37700, Brass }\end{array}$ \\
\hline Ball: & 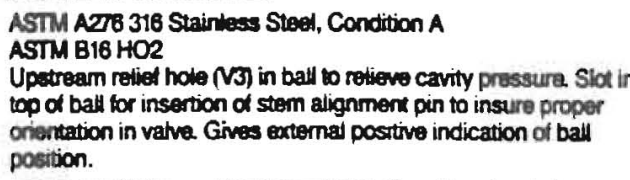 \\
\hline Stem: & 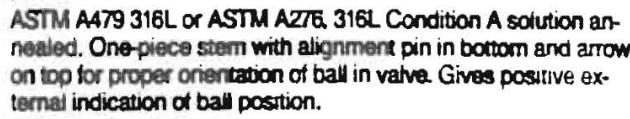 \\
\hline Seats: & Potyfill \\
\hline Body Seals: & "S" Gaskets (Tefion Coared 316 Stainless Steel) \\
\hline Stem Packing: & Chevron Packing (Tefion Style C-VH Pings) \\
\hline \multicolumn{2}{|c|}{ Packing Followers: ASTM A479 316L } \\
\hline Split Ring: & ASTM A4TY 316L \\
\hline $\begin{array}{l}\text { Split } \\
\text { Thrustbearing: }\end{array}$ & Pontill \\
\hline Extemals: & 300 Series Stainiess Steel \\
\hline \multirow{5}{*}{ End Connections: } & SE - Screwed Pipe Ends (NPT) (Dimenstons to ANSI B1.20.1) \\
\hline & $\begin{array}{l}\text { SW - Socket Weld Ends (Diameter and Depah of Bore to ANSI } \\
\text { B16.11) }\end{array}$ \\
\hline & $\begin{array}{l}\text { Swo. Socket Weld Ends for Q.D. Tubing to ASTM A269 (Stain. } \\
\text { loss Sleet Only) }\end{array}$ \\
\hline & $\begin{array}{l}\text { BW1 - Bum Wold Encts ior Schedule } 10 \text { Pipe (Dimensions to } \\
\text { ANS1 B16.25) (Stainiess Stoel Only) }\end{array}$ \\
\hline & $\begin{array}{l}\text { TE - Tube Ends (Sweat Ends tor Type K, L and M Copper } \\
\text { Tubing to ASTM B88) (Brass Only) }\end{array}$ \\
\hline $\begin{array}{l}\text { Temperatur } \\
\text { Range: }\end{array}$ & $-425 \circ \mathrm{F} 10+500^{\circ} \mathrm{F}$ \\
\hline $\begin{array}{l}\text { Maximum } \\
\text { Pressure: }\end{array}$ & 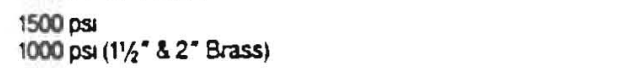 \\
\hline
\end{tabular}

\section{Wafer Valves and Flanged Valves with and without Extensions}

\begin{tabular}{|c|c|}
\hline Valve Sizes: & $3^{\circ}, 4^{\circ}$ and $6^{\circ}$ \\
\hline Style: & $\begin{array}{l}\text { Unidirectional Flow. Water/Flangeless and flanged Design } \\
\text { Blowout-Proot Stem }\end{array}$ \\
\hline Valve Ratings: & $\begin{array}{l}\text { Water - Fils between ANSI Class } 150 \text { and } 300 \text { Flanges } \\
\text { Flanged - ANSI Class } 150\end{array}$ \\
\hline $\begin{array}{l}\text { Body and } \\
\text { End Plug: }\end{array}$ & $\begin{array}{l}\text { ASTM A351 CF8M Stainiess Steet } \\
\text { ASTM B584 C83600 Brass (Water Onty) }\end{array}$ \\
\hline Ball: & $\begin{array}{l}\text { ASTM A240-316 or ASTM A312 GA TP.316 } \\
\text { Stainiess Steet } \\
\text { ASTM B584 C } 83600 \text { Brass (Water Onty) }\end{array}$ \\
\hline Stem: & ASTM A2T6 316 Stanless Steol Condrtion A \\
\hline Seats: & Polyfill \\
\hline Body Seal: & TFE-Virgin \\
\hline Stem Seals: & TFE - $15 \%$ Glass Filled \\
\hline $\begin{array}{l}\text { Centering } \\
\text { Washers: }\end{array}$ & $\begin{array}{l}\text { AISI } 316 \text { Stainiess Steor } \\
\text { ASTM B121 C34200 Brass (Wafer Onty) }\end{array}$ \\
\hline Thrustbearing: & TFE - $15 \%$ Glass Filed \\
\hline Follower: & AISI - 304 Stainiess Stoet \\
\hline Externais: & 300 Series Stainiess Steet \\
\hline
\end{tabular}

\section{C4 and C4 Diverter Valves without Extensions}

\begin{tabular}{|c|c|}
\hline Vhlve Slaes: & 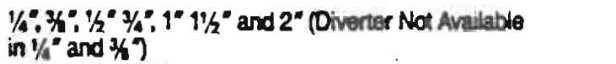 \\
\hline Styles: & $\begin{array}{l}\text { Unidirectional Fow, Threafiece Design } \\
\text { Top Entry Extended, One-Piece, Blowout-Proot Stem Diverter - } \\
\text { V., V2 Porting. }\end{array}$ \\
\hline Body: & $\begin{array}{l}\text { ASTM A743 CF3M. 316. Stainless Steal } \\
\text { ASTM B283 C3TTOC, Brass }\end{array}$ \\
\hline Prpe Ends: & $\begin{array}{l}\text { ASTM A743 CF3M, (3164) Stainless Steel } \\
\text { ASTM A743 CFBM, (316) Stainiess Steel } \\
\text { ASTM B283 C3T700, BFes }\end{array}$ \\
\hline Sents: & Pohrill \\
\hline Body Seais: & "S" Gaskets (Totron Coated 316 Stainless Steen) \\
\hline Externalia: & 300 Series Stainless Stad \\
\hline \multirow[t]{5}{*}{ End Connections: } & SE - Scremed Pipo Ends (NPT) (Dimensions to ANSI B1.20.1) \\
\hline & $\begin{array}{l}\text { Sw - Socker Weid Ends (Diameter and Depth o Bore to ANSI } \\
\text { Biai1) }\end{array}$ \\
\hline & $\begin{array}{l}\text { SWo - Socteat Weld Ends for Q.D. Tubing to ASTM A269 (Stain- } \\
\text { Steet Only) }\end{array}$ \\
\hline & $\begin{array}{l}\text { BW1 - Butt Weld Ends for Schedule to Pipe (Dimensions to } \\
\text { ANSI B16.25) (Stainless Steel Only) }\end{array}$ \\
\hline & $\begin{array}{l}\text { TE - Tube Ends (Swaat Ends for Fype K, L and M Copper } \\
\text { Tubing io ASTM B88) (Brass Onty) }\end{array}$ \\
\hline Ball: & $\begin{array}{l}\text { ASIM A276 } 316 \text { Stainiess Sted, Condition A } \\
\text { ASTM B16 HO2 } \\
\text { Upstream reliet hole (V3) in ball to relieve cavity pressure. }\end{array}$ \\
\hline Stem: & $\begin{array}{l}\text { ASTM A276 } 316 \text { Stainless Steel, Condition A } \\
\text { ASTM B16 HO2 }\end{array}$ \\
\hline Packing follower: & AISI 3:6L Stainiess Steel \\
\hline Stem Seal: & TFE - $15 \%$ Glass Filled \\
\hline Thrustbeanng: & Polytill \\
\hline
\end{tabular}

\section{General Specifications}

Shutott:

The valve exceeds the tightness requirements of ISA SP79 for Lovel II.10 SCFH per inch of vatve (10) 100 psi Helium and $-320 \%$ F.

Packaging: End connectons are capped or plugged, and valves are placed in 4 mil thick (minimum) potyettmylene bags and heat sealed.

Operation: Lover handie, pneumanc or electric actuators.

Cleaning: Cleanng tor all vaves is based on the axygen cleaning procedures of Linde Division Spec, GS38 Body, ball, stem and pipe onds degreased.

Lubrication: Molycoar 321P.

Aceembly: Assembled in a Class 100 emvronment per Federal Standard 2098

Testing: Valves are ory tested. internally pressurized with 80 psi Helium. checked with hand-hold helium leak detector (Helium Mass Spectrometer) sonstive $101 \times 10^{-9} \operatorname{ccs} s e c$ 
Body Temperature vs Bonne: -emperature $1 / 4=2 " 3$ Piece and Diverter Valves with Extension Ambient Temperature $75^{\circ} \mathrm{F}$

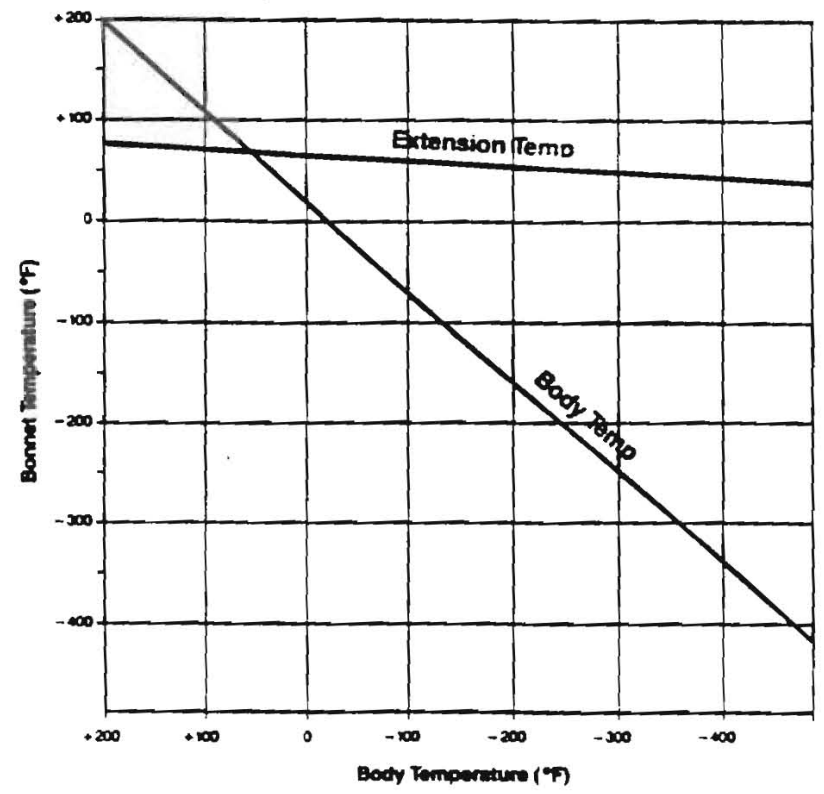

Pressure Torque Curves - Polyfill Seats $1 / 4^{\prime \prime}-2^{\prime \prime}$ CA and CA Diverter Valves

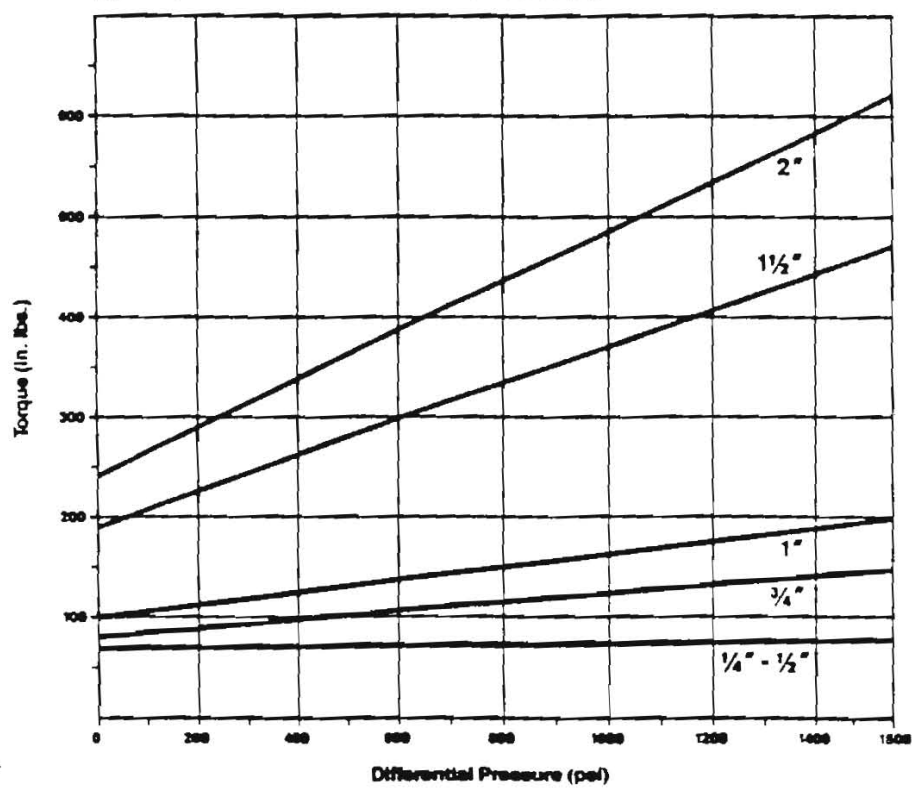

Pressure/Temperature Ratings

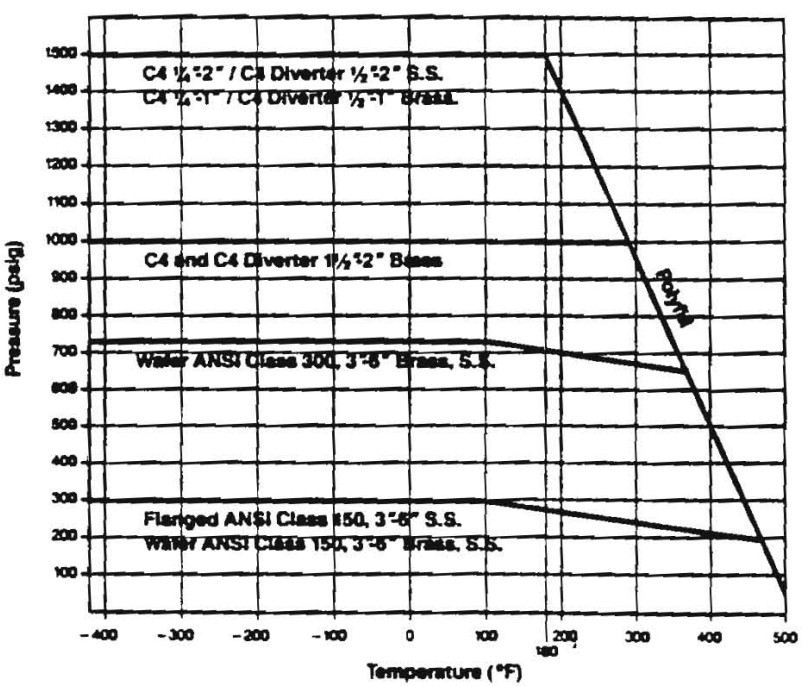

Pressure Torque Curves - Polyfill Seat $3^{\prime \prime}-6^{\prime \prime}$ Wafer and Flanged Valves

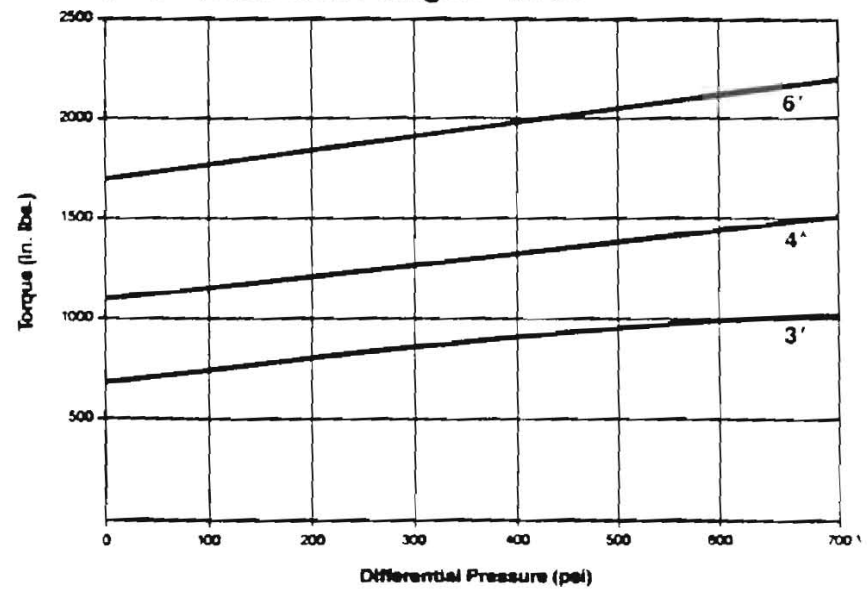

Flow Coefficient

Cv values and equivalent lengths of pipe

\begin{tabular}{|c|c|c|c|c|c|c|c|c|}
\hline & \multicolumn{8}{|c|}{ 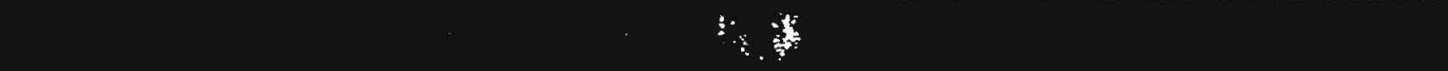 } \\
\hline $1 / 4=-1 / 2$ & 8 & 3 & & & 31 & 19.6 & & \\
\hline $3 / 3=$ & 12 & 5 & & & 63 & 30.4 & & \\
\hline $1^{\prime \prime}$ & 32 & 10 & & & 3.1 & 27.8 & & \\
\hline $11 / 2 "$ & 82 & 24 & & & 4.3 & 435 & & \\
\hline $2^{\prime \prime}$ & 120 & 36 & & & 75 & 22.7 & & \\
\hline $3^{\circ}$ & & & 350 & 350 & & & 7.1 & 7.1 \\
\hline $4^{\prime \prime}$ & & & 720 & 720 & & & 6.9 & 6.9 \\
\hline $6^{\prime \prime}$ & & & 1020 & 1020 & & & 20.4 & 20.4 \\
\hline
\end{tabular}




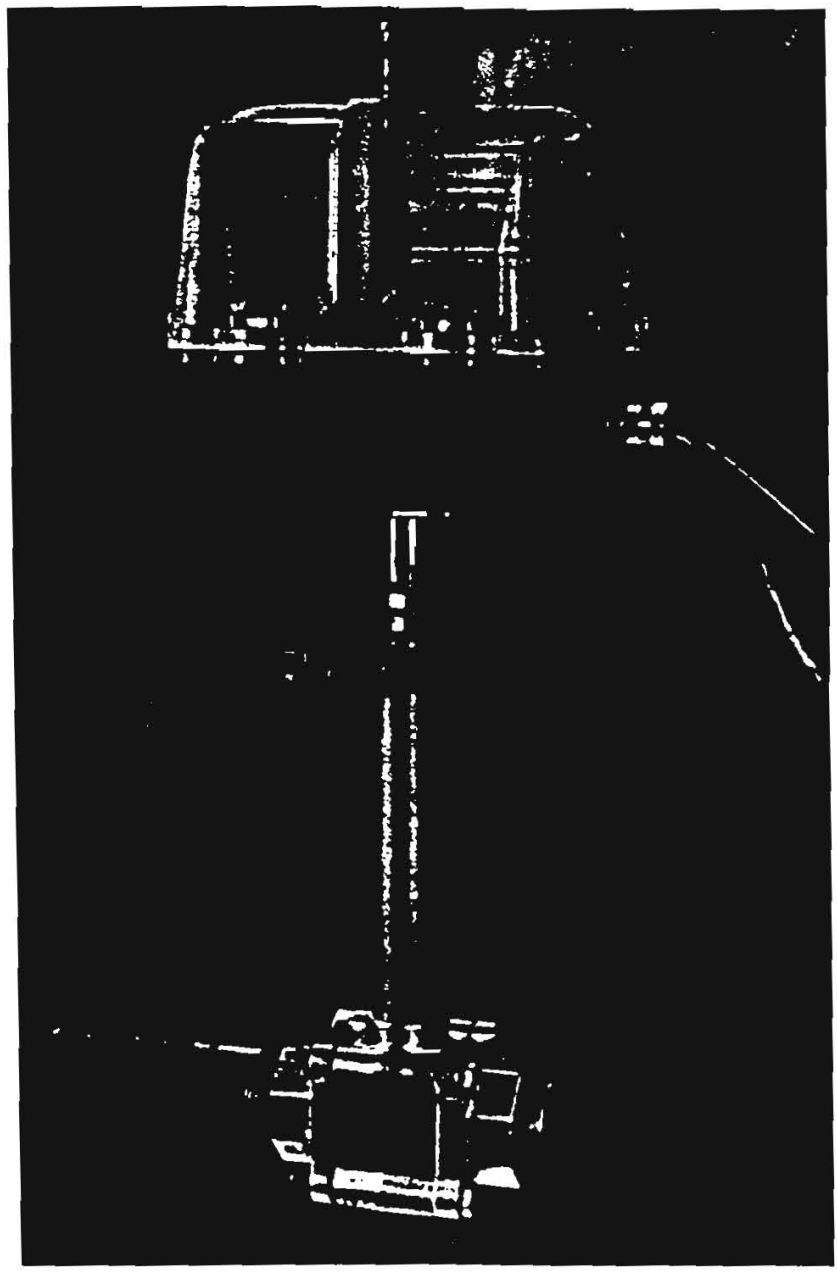

\section{AUTOMATION}

PNEUMATIC AND

\section{ELECTRIC CONTROLS}

Easy automation is assured by our Series 39 pneumatic or Series 75 electric actuators. Both are backed by our exclusive two-year warranty. The Series 39 actuator is the toughest and most versatile rotary actuator available. Positioners (including electro-pneumatic), fail-safe feature, and mechanical and proximity limit switches provide ON/OFF or proportional control to your system with the feedback you require. Refer to Bulletin No. PB302.

Mount a Series 75 electric actuator and you have a high performance control valve package specifically designed for computer or PLC control. For ON/OFF or proportional control, the Series 75 can take a digital or analog signal. A variety of options allow you to select the performance criteria and feedback information you desire. The Series 75 is available with NEMA I, IV, VII or IX enclosures. Refer to Bulletin No. PB730.

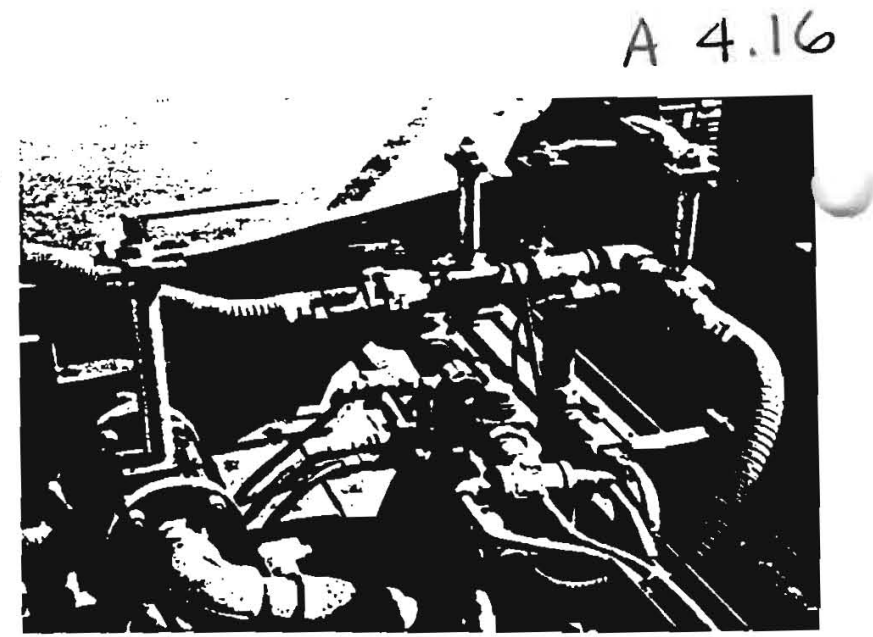

\section{APPLICATIONS}

Over the Road LNG-LPG Trailers

Terminal Unloading Stations

High Purity Cryogenic/Gas Systems

LNG Storage and Distribution

$\mathrm{CO}_{2}$ and Nitrogen Injection for Enhanced Oil Recovery

Over the Road $\mathrm{CO}_{2}, \mathrm{LN}_{2}$ Food Carners

Petroleum Refining Unleaded Gasoline (Gas Treatment Skids)

Lyophilization Systems

Air Separation Plants

Liquid and Gaseous Oxygen for Steel Production

Inerting and Heat Treatment
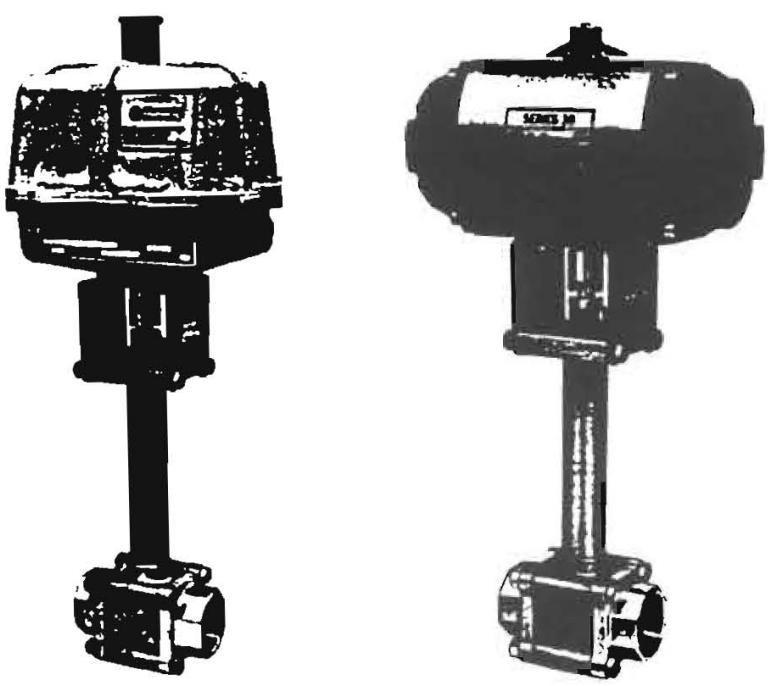
Worcester Controls

A BTR Company +
Series 39 Pneumatic Actuator

Twin piston, double rack and pinion pneumatic actuator offers long cycle life for rotary applications

PB-302-24

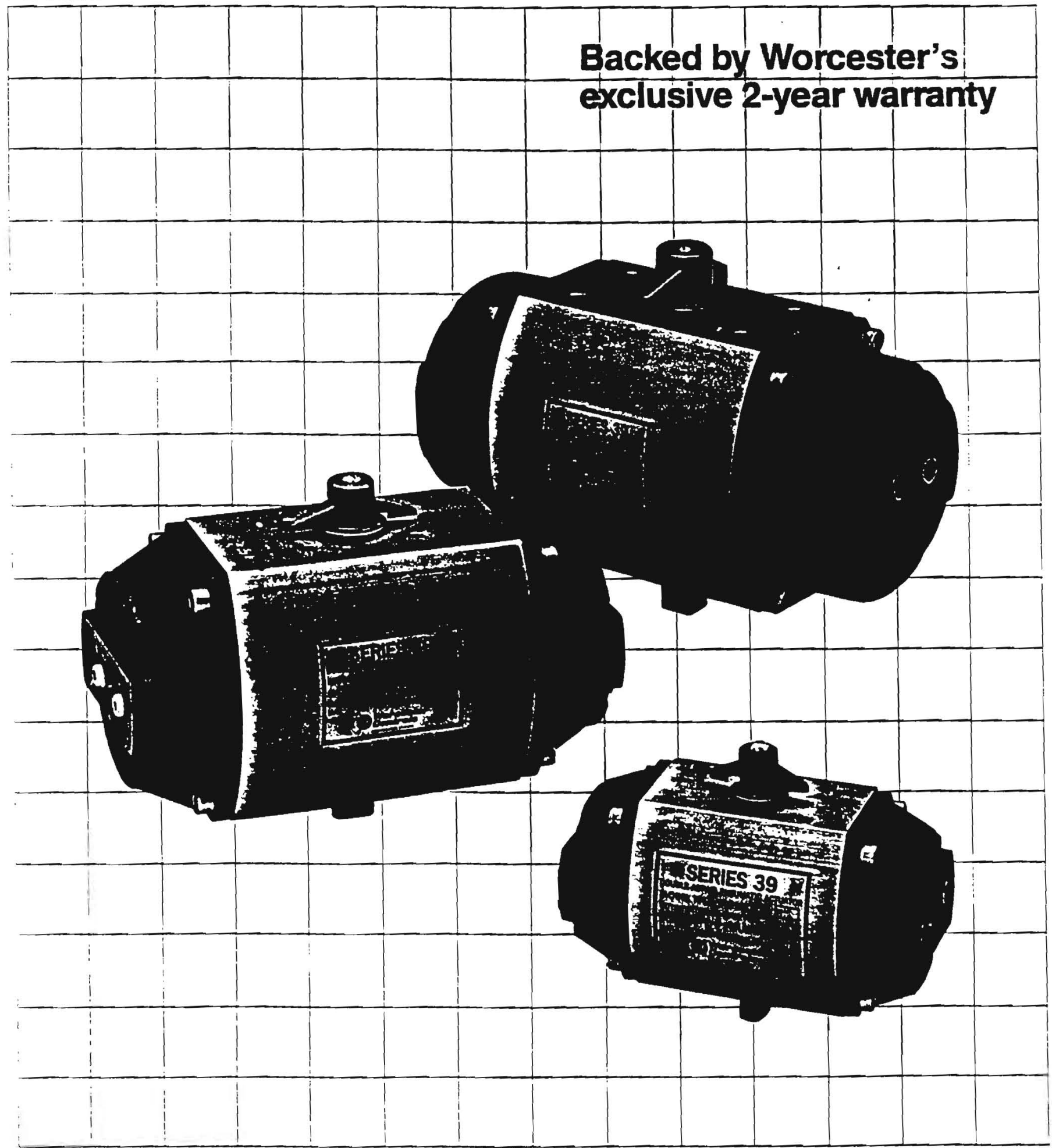




\section{Series 39 Fneumatic Aetuator}
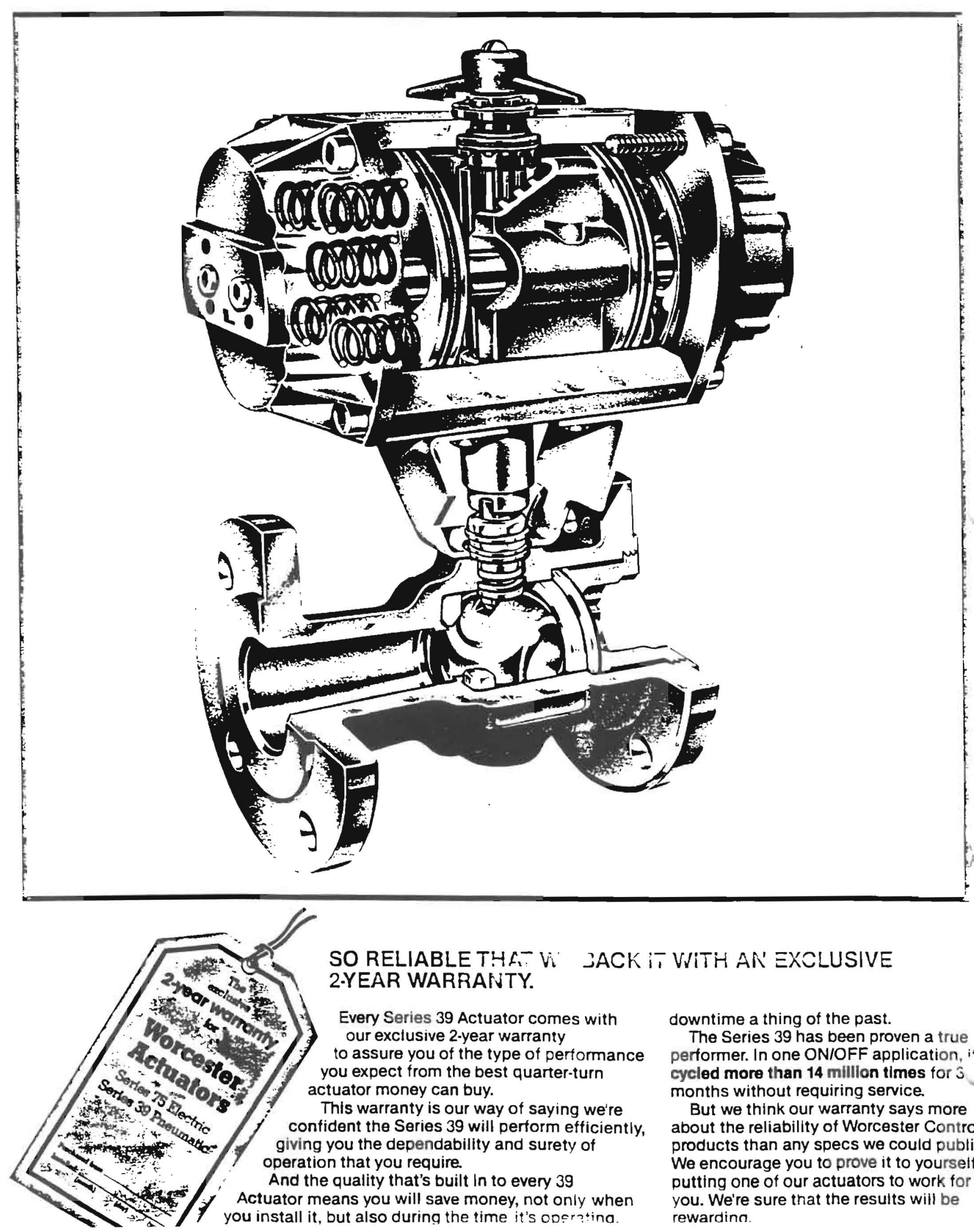

downtime a thing of the past.

The Series 39 has been proven a true performer. In one ON/OFF application, i* cycled more than 14 million times for 3 months without requiring service.

But we think our warranty says more about the reliability of Worcester Controls products than any specs we could publish. We encourage you to prove it to yourself by putting one of our actuators to work for you. We're sure that the results will be rewardinn. 
Design Features

\section{COMPACT DOUBLE-PISTON DESIGN}

develops extremely high torque output from a short stroke. Plant air from a single connection is valved through ports in sliding piston guide rods to inner and outer air chambers alternately. Both pistons thus provide power for both opening and closing actions. Each piston is affixed to, centered, and guided by a moving stainless steel guide rod, operating in new, lower friction acetal resin beanings, eliminating side loading of shaft.

RACK AND PINION power transfer with constant balanced-force engagement minimizes backlash and provides balanced piston loading for equal wear. Gear teeth cut across the full face of pistons and pinion provides massive engagement with minimum tooth loading. Internal piston bearings eliminate side loads on " $O$ " rings, extending life.

\section{RUGGED CONSTRUCTION - CORROSION}

RESISTANT body of the Series 39 actuator is aluminum. Coro-lube coated, both inside and outside, for strength and corrosion resistance. Increased metal thickness of body improves life of shaft bearing. Pistons are aluminum for strength and corrosion resistance. End caps are aluminum, Coro-lube coated. Integral pinion and shaft is steel, plated for corrosive resistance. Stainless steel end cap screws are used.

Coro-lube is a nickel-acetate filled coating. It provides greater resistance to wear and corrosion and also provides increased lubricity on internal surfaces.

EXTENDED TOP SHAFT provides visual indication of valve position and permits the use of optional accessories. such as top-mounted limit switches. a pneumatic positioner or means for manually overriding the actuator. It aiso allows the operation of two valves with a single actuator. The extended shaft simplifies mounting to virtually any rotary valve.

\section{LOWER INTERNAL FRICTION results from} Coro-lube coatings, low coefficient-of-friction acetal resin bearings, and polished piston guide rods. Operating speed and torque output are improved as a result of requiring a very small pressure differential to produce motion.

SPRING RETURN models are available for applications requiring the valve to move to either a closed or opened position on failure of electrical or air supplies. Depending on air supply pressure, the multi-spring design provides sufficient torque to cycte the valve in the event of such failures. The failure mode can be changed simply by inverting the actuator, changing the valve position and remounting the actuator.

In corrosive or contaminating environments, the spring chamber port can be piped to a cleaner environment to reduce corrosion inside the actuator.

CONTROL BLOCK provides extremely fine independently adjustable speed control for both opening and closing strokes of double acting units. Only the spring stroke is adjustable on spring return units. Available with General Purpose NEMA I, Watertight NEMA IV, Hazardous Environment NEMA VII (UL \& CSA listed for Class I, Group D) and NEMA IX (Class H, Groups E, F \& G) solenoids for 24, 115 and $230 \mathrm{~V} A C$ and 6,12 and $24 \mathrm{~V} \mathrm{DC}$ operation, NEMA IV and VII solenoid in one enctosure is also available. in the event of electrical failure or when local operation is desired, the double-acting solenoid may be overridden by manual operation of the control block.

\section{Operatinc Secjense}

Double Acting

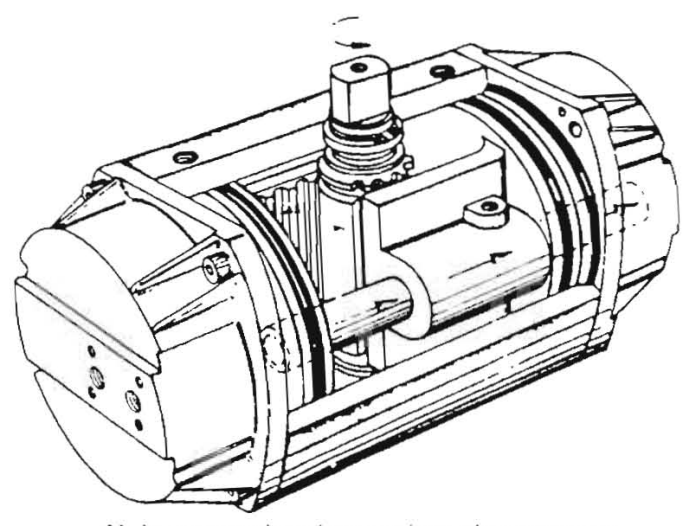

Air in center chamber pushes pistons apart.

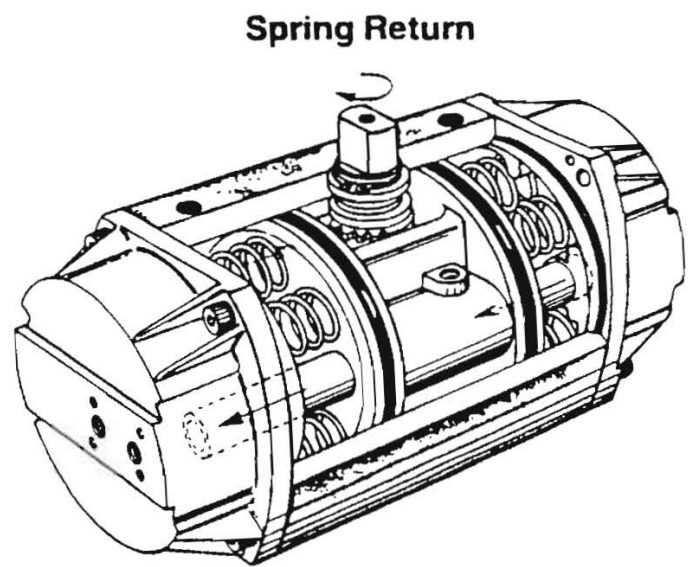

Springs in end caps of spring return actuator push pistons back together for fail safe operation.
In a double acting actuator, air enters the center chamber to push the pistons apart. opening the valve. When closing the valve, the double acting actuator relies on air supplied on the outside of each piston to push the pistons together again.
In a spring return actuator, air is supplied through the cemer chamber to push the pistons apart. Springs located in the end caps of the actuator relax to automatically push the pistons back together, thus closing the valve. These springs a!so provide a dependable ?ail safe closure in the event of 


\section{Engimeering Data (Revision 3)}

\begin{tabular}{|c|c|c|c|c|c|c|c|c|c|c|c|}
\hline \multirow{2}{*}{$\begin{array}{l}\text { nis-2 } \\
\text { Mopa } \\
\text { Ma }\end{array}$} & \multicolumn{4}{|c|}{ 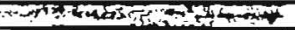 } & \multicolumn{7}{|c|}{ 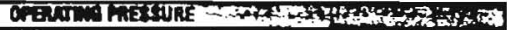 } \\
\hline & 30 & 40 & $\begin{array}{r}50 \\
?:- \\
\end{array}$ & \begin{tabular}{l|}
60 \\
$\therefore$
\end{tabular} & $\begin{array}{c}70 \\
14 n_{i}\end{array}$ & $\begin{array}{l}80 \\
5 \therefore 1 \\
\end{array}$ & 90 & $\begin{array}{c}100 \\
\therefore \therefore:\end{array}$ & $\begin{array}{c}110 \\
\therefore=:\end{array}$ & $\begin{array}{c}120 \\
: 3: 1 \\
0\end{array}$ & $\begin{array}{l}\text { PSI } \\
8::\end{array}$ \\
\hline & $\begin{array}{l}80 \\
5 ?\end{array}$ & $\begin{array}{l}125 \\
\because .\end{array}$ & $\begin{array}{l}160 \\
18:\end{array}$ & $\begin{array}{l}200 \\
22 \vdots\end{array}$ & $\begin{array}{l}245 \\
i 7 i\end{array}$ & $\begin{array}{l}270 \\
30 \lesssim\end{array}$ & $\begin{array}{l}310 \\
35:\end{array}$ & $\begin{array}{l}350 \\
39=\end{array}$ & $\begin{array}{l}385 \\
i 35\end{array}$ & $\begin{array}{l}425 \\
480\end{array}$ & $\begin{array}{c}\text { in. }+\mathrm{b} \text {. } \\
\mathrm{Nim}\end{array}$ \\
\hline & $\begin{array}{l}155 \\
\because-i\end{array}$ & 240 & 300 & $\begin{array}{l}370 \\
\vdots 1: \\
\end{array}$ & $\begin{array}{l}460 \\
5 ?: 2 \\
\end{array}$ & $\begin{array}{l}510 \\
57 \pi \\
10\end{array}$ & $\begin{array}{l}560 \\
655 \\
\end{array}$ & 650 & $\begin{array}{l}725 \\
8 i^{\circ} \\
\end{array}$ & $\begin{array}{l}790 \\
\text { ge . . } \\
\end{array}$ & $\begin{array}{l}\text { in. }+10 . \\
\text { An: }\end{array}$ \\
\hline 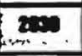 & $\begin{array}{c}285 \\
3 \\
\end{array}$ & $\begin{array}{c}435 \\
\vdots-1 . \\
\end{array}$ & $\begin{array}{l}545 \\
i 11 \mathrm{i} \\
\end{array}$ & $\begin{array}{l}680 \\
\text { î́:- }\end{array}$ & $\begin{array}{l}840 \\
94 ? ?\end{array}$ & $\begin{array}{l}935 \\
100^{\circ}\end{array}$ & $\begin{array}{l}1070 \\
12 .\end{array}$ & $\begin{array}{l}1200 \\
3 .\end{array}$ & $\begin{array}{l}1330 \\
155^{\prime}\end{array}$ & $\begin{array}{l}1460 \\
16:\end{array}$ & in.tib. \\
\hline & 590 & $\begin{array}{l}785 \\
\text { 列: }\end{array}$ & 980 & $\begin{array}{l}1280 \\
13^{\circ}\end{array}$ & $\begin{array}{l}1375 \\
15 \div\end{array}$ & $\begin{array}{l}1570 \\
177^{-}\end{array}$ & $\begin{array}{l}1770 \\
n:\end{array}$ & 1965 & $\begin{array}{l}2160 \\
12:\end{array}$ & $\begin{array}{l}2355 \\
2 \because i\end{array}$ & in.the \\
\hline & $\begin{array}{c}790 \\
! \\
\end{array}$ & 1200 & 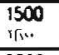 & $\begin{array}{l}1860 \\
: 1 " . . \\
\end{array}$ & $\begin{array}{l}2305 \\
26 ! \\
\end{array}$ & $\begin{array}{l}2580 \\
2 c^{\circ} \\
\end{array}$ & $\begin{array}{l}2935^{\circ} \\
33^{\circ} \\
\end{array}$ & $\begin{array}{l}3290 \\
37 \\
\end{array}$ & $\begin{array}{l}3645 \\
\vdots ! \\
\end{array}$ & $\begin{array}{l}4000 \\
i^{\prime} \\
\end{array}$ & 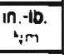 \\
\hline & $\begin{array}{l}2220 \\
? !\end{array}$ & $\begin{array}{l}2975 \\
35 . \\
\end{array}$ & $\begin{array}{l}3900 \\
44^{\circ}\end{array}$ & $\begin{array}{l}4800 \\
5 \div 2 \\
\end{array}$ & $\begin{array}{l}5600 \\
f 3:\end{array}$ & $\begin{array}{l}6400 \\
\therefore \cdots\end{array}$ & $\begin{array}{l}7200 \\
4:-\end{array}$ & $\begin{array}{l}8000 \\
4 i:-\end{array}$ & $\begin{array}{l}8800 \\
92::\end{array}$ & $\begin{array}{l}9600 \\
\cdots ! ? ?\end{array}$ & $\begin{array}{l}\text { In. }-1 \mathbf{b} \\
\mathrm{N}-\mathrm{-}\end{array}$ \\
\hline $\begin{array}{l}4007 \\
1061\end{array}$ & $\begin{array}{l}3110 \\
i^{2}\end{array}$ & $\begin{array}{l}4900 \\
5=-\end{array}$ & $\begin{array}{l}6200 \\
70^{\circ}\end{array}$ & $\begin{array}{l}76000 \\
0.4 \text { in } \\
\end{array}$ & ירי & $\begin{array}{l}10230 \\
\cdots 1\end{array}$ & $\begin{array}{l}11500 \\
1 ? n^{\prime \prime}\end{array}$ & $\begin{array}{l}12800 \\
\therefore \therefore\end{array}$ & $\begin{array}{l}14200 \\
: 020^{\prime}\end{array}$ & $\begin{array}{l}15500 \\
7+c .\end{array}$ & $\begin{array}{c}\mathrm{in} \cdot \dot{10} . \\
\mathrm{Nm}\end{array}$ \\
\hline $\begin{array}{l}4599 \\
797.1\end{array}$ & 9000 & 12700 & $\begin{array}{l}16100 \\
: 8 ?^{\circ} \\
\end{array}$ & $\begin{array}{l}19500 \\
7 ? 0 \\
7 ?\end{array}$ & $\begin{array}{l}22700 \\
\end{array}$ & $\begin{array}{l}26000 \\
\therefore .4 . \\
\end{array}$ & $\begin{array}{l}29400 \\
? 20 \\
\end{array}$ & 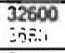 & $\begin{array}{l}360000 \\
-10 \div .1 \\
\end{array}$ & $\begin{array}{l}39500 \\
\text { isi? }\end{array}$ & $\begin{array}{c}1 .+10 . \\
: m \\
: m\end{array}$ \\
\hline 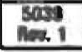 & $\begin{array}{l}13145 \\
\therefore .6\end{array}$ & 19000 & 24000 & $\begin{array}{l}29000 \\
i \div ?\end{array}$ & $\begin{array}{l}340000 \\
\text { ?H: }\end{array}$ & $\begin{array}{l}40000 \\
\therefore \because 4\end{array}$ & $\begin{array}{l}450000 \\
508: 1\end{array}$ & $\begin{array}{l}50000 \\
5 E \therefore .\end{array}$ & $\begin{array}{l}55000 \\
52^{\circ}\end{array}$ & $\begin{array}{l}600000 \\
n i ?\end{array}$ & $\begin{array}{c}\pi-10 . \\
1 \Gamma 1 \\
\Gamma 1\end{array}$ \\
\hline
\end{tabular}

STROKE TIME (seconds) (double acting)*

\begin{tabular}{|c|c|c|}
\hline-1008 & $\begin{array}{l}\text { Monum } \\
\text { (uwconden) }\end{array}$ & $\begin{array}{l}\text { wirn maximum } \\
\text { sries commat }\end{array}$ \\
\hline 1009 & Less than 1 & 10 \\
\hline 1539 & Less than 1 & 15 \\
\hline$=2039$ & 1 & 15 \\
\hline-2539. & $2 \cdot 3$ & 18 \\
\hline$\therefore 3009 \div$ & 3.4 & 20 \\
\hline$\because 359 \div$ & $4 \cdot 5$ & 25 \\
\hline 4009 Rov. 1 . & $5-6$ & 30 \\
\hline 4539 Aer.: 1 & $10 \cdot 12$ & 40 \\
\hline 5039 A Av. 1. & $12-14$ & 60 \\
\hline
\end{tabular}

NOTE: These figures are meant as an indication of obtainable speeds only. For more precise figures for any particular application, contact Worcester Controls. Faster speeds are obtainable, if required, by using additional control equipment.

werega imes under $50 \%$ losd conditione. 80 pes (with standerd sowenom).

Air Consumption per Stroke (free air)

\begin{tabular}{|c|c|c|c|c|c|c|c|c|c|}
\hline , & 1000 & 1500 & 2000 & 7539. & 300 & 350 & 4000 & 4500 & 5000 \\
\hline BOPEO $=$ & 0.04 & 0.08 & 0.16 & 0.28 & 0.43 & 0.9 & $\begin{array}{l}1.26 \\
35 \\
35\end{array}$ & $\begin{array}{l}3.1 . \\
57 .\end{array}$ & 5.5 \\
\hline $\begin{array}{l}\text { b CLOSE } \\
\text { (0.A. onvy) }\end{array}$ & 0.05 & 0.09 & 0.17 & 0.3 & 0.47 & $\begin{array}{c}1.27 \\
j !\end{array}$ & 1.43 & 46 & 7.0 \\
\hline
\end{tabular}

\section{ACTUATOR WEIGHTS *}

\begin{tabular}{|c|c|c|}
\hline \multirow{2}{*}{ 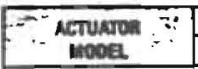 } & 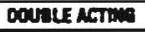 & 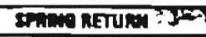 \\
\hline & Let (ln) & $=\operatorname{Lan}(x) \sim-2-1$ \\
\hline$+1009 \quad \therefore$ & $3(1.3)$ & $3.5(1.6)$ \\
\hline$\because \quad \because 1539 \because \ldots$ & $6(2.7)$ & $7(3.1)$ \\
\hline r. $\quad 2009 \ldots$ & $10(4.5)$ & $12(5.5)$ \\
\hline$-\because 2539 \cdots$ & $16.25(7.4)$ & $18.5(8.4)$ \\
\hline $3030-15$ & $246(11)$ & $27(12)$ \\
\hline$-3530-2 \div$ & $58(26)$ & $65(30)$ \\
\hline 4039 fow. 1 ..... & $70(32)$ & $80(36)$ \\
\hline 4539 Rov. $1^{-1}$ & $213(97)$ & $253(115)$ \\
\hline$=5039$ Rov. $1=$ & $304(138)$ & $355(161)$ \\
\hline
\end{tabular}

mithoul solenowe

\section{SIZING}

Determine appropriate valve torque requirements from valve literature. For double-acting actuators, select the actuator whose torque output at available air supply exceeds breakaway torque requirements of the valve. For detailed instructions, consult Worcester Controls Actuator Sizing Manual, bulletin ASM.

For fail-closed. spring-return actuators, select the appropriate size actuator whose torque output at end of spring stroke (at available air supply) is sufficient to close the valve.

For fail-open spring-return actuators, select appropriate actuator whose torque output at the end of the air stroke is sutficient to close the valve. For

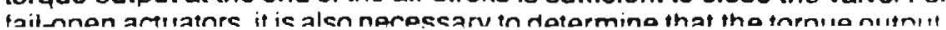

TORQUE OUTPUT — Spring Return Actuator

\begin{tabular}{|c|c|c|c|c|c|c|c|c|c|}
\hline \multirow{2}{*}{\multicolumn{2}{|c|}{ tor }} & \multicolumn{8}{|c|}{ 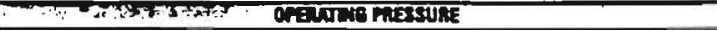 } \\
\hline & & \multicolumn{2}{|c|}{$12 n$} & \multicolumn{2}{|c|}{$\begin{array}{c}50 \\
(3.4) \\
\end{array}$} & 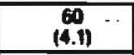 & $\cdots{ }_{(4.1)}^{70}$ & $\begin{array}{c}\infty \\
(5.4)\end{array}$ & $\begin{array}{l}\text { PSI } \\
\text { pain }\end{array}$ \\
\hline \multicolumn{2}{|c|}{ Na of Springs } & \multicolumn{2}{|c|}{4.4} & \multicolumn{2}{|c|}{. 6} & 8 & .8 & 10 & \\
\hline & STRONE & START & END & START & END & START END & START END & START END & \\
\hline \multirow{2}{*}{ 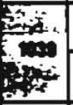 } & $\therefore N A$ & $\begin{array}{l}85 \\
96\end{array}$ & $\begin{array}{l}60 \\
5=\end{array}$ & $\begin{array}{l}105 \\
\cdots 5\end{array}$ & $\begin{array}{l}60 \\
11 .\end{array}$ & $\begin{array}{l}125 \\
\because::\end{array}$ & $170 \quad 120$ & $175 \quad 95$ & in. 16 . \\
\hline & Spraig & & $\begin{array}{l}35 \\
4 \\
\end{array}$ & $\begin{array}{l}95 \\
10\end{array}$ & $\begin{array}{l}55 \\
6^{n}\end{array}$ & 125 & 125 & 160 & in.to. \\
\hline \multirow{2}{*}{ tis } & ; & $\begin{array}{l}130 \\
\because:- \\
\end{array}$ & $\begin{array}{l}85 \\
95 \\
9.7 \\
\end{array}$ & $\begin{array}{l}200 \\
.2 \mathrm{~F} \\
\end{array}$ & $\begin{array}{l}125 \\
\because \therefore \\
\end{array}$ & 240 & \begin{tabular}{ll}
260 & 155 \\
\hdashline$-\therefore$ \\
\end{tabular} & $\begin{array}{ll}325 & 190 \\
35 & \therefore \\
\end{array}$ & 2.10 \\
\hline & spining & $\begin{array}{l}105 \\
19 \\
\end{array}$ & $\begin{array}{l}65 \\
73\end{array}$ & $\begin{array}{l}165 \\
186\end{array}$ & $\begin{array}{l}105 \\
\because \cdots\end{array}$ & $\begin{array}{ll}220 & 145 \\
\therefore: & \because .\end{array}$ & \begin{tabular}{|ll}
220 & 145 \\
$\therefore$ & $\mathrm{n}:$
\end{tabular} & \begin{tabular}{|ll}
200 & 185 \\
$3 ! !$ & $\cdots$
\end{tabular} & in. Ath. \\
\hline \multirow{2}{*}{ 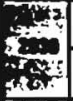 } & Nints & $\begin{array}{l}3000 \\
33 \div 0\end{array}$ & $\begin{array}{l}240 \\
\therefore:\end{array}$ & $\begin{array}{l}340 \\
35: 4 \\
\end{array}$ & 235 & $\begin{array}{ll}415 & 280 \\
\text { iri; } & :-\end{array}$ & $\begin{array}{ll}575 & 40 \\
\vdots= & \vdots- \\
\end{array}$ & $\begin{array}{ll}6000 & 360 \\
\text { ni. } & \because \\
\end{array}$ & n. \\
\hline & $\sin _{x \rightarrow \infty}$ & $\begin{array}{l}190 \\
7^{\circ}: \\
\end{array}$ & $\begin{array}{l}125 \\
1 .\end{array}$ & $\begin{array}{l}300 \\
33 \&\end{array}$ & 195 & $\begin{array}{c}265 \\
\ddots \\
\end{array}$ & 265 & $505 \quad 335$ & in - to. \\
\hline \multirow{2}{*}{ 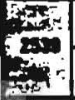 } & Non: & $\begin{array}{l}560 \\
53,\end{array}$ & $\begin{array}{l}400 \\
1^{2} 1\end{array}$ & 600 & 2350 & $\begin{array}{l}730 \\
\vdots\end{array}$ & 655 & 980 & in. 10 . \\
\hline & Spenga & $\begin{array}{l}345 \\
.3411\end{array}$ & 210 & $\begin{array}{l}540 \\
\therefore \because 6 .\end{array}$ & $\begin{array}{l}330 \\
3^{-}\end{array}$ & $\begin{array}{l}450 \\
: \quad .\end{array}$ & 720 & 575 & in. 10. \\
\hline \multirow{2}{*}{ 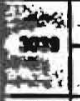 } & $\therefore$ An & $\begin{array}{l}840 \\
\text { is: }=\end{array}$ & $\begin{array}{l}610 \\
69: 1\end{array}$ & $\begin{array}{l}965 \\
10.4\end{array}$ & $\begin{array}{l}600 \\
\therefore \therefore .\end{array}$ & $\begin{array}{ll}1130 & 690 \\
\because & \ddots\end{array}$ & $\begin{array}{ll}1575 & 1145 \\
\cdots & !\end{array}$ & $\begin{array}{ll}1650 & 920 \\
4 & 9\end{array}$ & 10.10 \\
\hline & SProng & $\begin{array}{l}560 \\
r ?: 3\end{array}$ & $\begin{array}{l}340 \\
29+1\end{array}$ & $\begin{array}{l}870 \\
4 \div \div: 3\end{array}$ & 535 & $\begin{array}{ll}1160 & 730 \\
\because & \end{array}$ & 1160 & $1470 \quad 920$ & in. 1 to. \\
\hline \multirow{2}{*}{ Ther } & 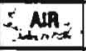 & 2100 & 1470 & $\begin{array}{l}2360 \\
3^{-1}-\end{array}$ & $\begin{array}{l}1450 \\
\cdots: \\
\end{array}$ & $\begin{array}{l}2850 \\
\because\end{array}$ & $\begin{array}{ll}3570 & 2615 \\
\vdots\end{array}$ & $\begin{array}{ll}3850 & 2210 \\
\vdots & \\
\end{array}$ & in. 10. \\
\hline & SPFina & 1330 & $\begin{array}{l}850 \\
\cdots .\end{array}$ & $\begin{array}{l}2070 \\
27.1\end{array}$ & 1330 & 2770 & $2770 \quad 1815$ & $\begin{array}{l}3500 \quad 2300 \\
\ldots . .\end{array}$ & in. 10 . \\
\hline \multirow{2}{*}{ in. of } & Springs & $\because \because$ & $12-2$ & & 8 & 16 & 22 & 24 & \\
\hline & $\therefore N R$ & $\begin{array}{l}3400 \\
2: 2\end{array}$ & $\begin{array}{l}1150 \\
\cdots \\
\end{array}$ & $\begin{array}{l}4250 \\
58: 1\end{array}$ & $\$ 150$ & 5400 & 2050 & 2830 & in +10 \\
\hline $\begin{array}{l}x+1 \\
\sin \\
2 x\end{array}$ & SPRTNG & 3850 & 1500 & \begin{tabular}{|l|}
5050 \\
$\therefore 7$ \\
\end{tabular} & 1950 & $\begin{array}{ll}5700 & 2200 \\
n+: & \vdots \\
\end{array}$ & 2750 & 3000 & in. 10 . \\
\hline \multirow{2}{*}{$\begin{array}{l}x+1- \\
4590 \\
\ln 1\end{array}$} & An & 8700 & $\begin{array}{l}4000 \\
i \\
\end{array}$ & $\begin{array}{l}10600 \\
? ; i K \\
\end{array}$ & $\begin{array}{l}4300 \\
\therefore \\
\end{array}$ & $\begin{array}{ll}13200 & 5900 \\
\because: & \cdots \\
\end{array}$ & 149006100 & $\begin{array}{l}17600 \quad 8000 \\
\text {. }\end{array}$ & $\ln .10$ \\
\hline & SPRTHG & 8300 & $\begin{array}{l}4000 \\
\because:\end{array}$ & $\begin{array}{ll}11800 \\
1 . \cdots\end{array}$ & 5500 & $\begin{array}{ll}15600 & 6300 \\
\therefore\end{array}$ & $\begin{array}{ll}16600 & 7800 \\
\cdots & \\
\end{array}$ & 180008400 & $\ln \cdot 10$ \\
\hline \multirow{2}{*}{5009} & NA & $\begin{array}{l}12500 \\
\because \cdots \\
\end{array}$ & 6000 & $\begin{array}{l}15500 \\
15 i^{\prime}\end{array}$ & 6000 & $19500 \quad 8500$ & $\begin{array}{ll}21800 & 8000 \\
\because & \end{array}$ & $26500 \quad 11500$ & $\ln \cdot 10$ \\
\hline & SPRinG & 13000 & 6500 & $\begin{array}{l}18000 \\
110\end{array}$ & $\begin{array}{l}8500 \\
3 .\end{array}$ & $20500 \quad 9500$ & $26000 \quad 12200$ & $28500 \quad 13500$ & $\ln \cdot 10$ \\
\hline
\end{tabular}

Air Flow Requirements

\begin{tabular}{|c|c|c|}
\hline$\therefore, \quad$ Actas & $\because$ UR. U. & $\therefore \quad \begin{array}{l}0=0 \\
49.8=\end{array}$ \\
\hline $\begin{array}{l}1039.1539 \\
2039.2539\end{array}$ & $1 / 8=$ Tubung & $1 / 4^{\circ}$ luang \\
\hline $\begin{array}{r}3539.4039 \\
4539.5039 \\
0\end{array}$ & $1 / 4^{-}$Tubang & $1 / 2=$ Tubxing \\
\hline
\end{tabular}

\section{Operating Conditions}

\begin{tabular}{|c|c|}
\hline \multirow[t]{2}{*}{$\begin{array}{l}\text { Pressure } \\
\text { Range }\end{array}$} & $\begin{array}{l}\text { 30 - } 120 \text { PSI Double Acting } \\
40-120 \text { PSI All Spring Return Versions" }\end{array}$ \\
\hline & 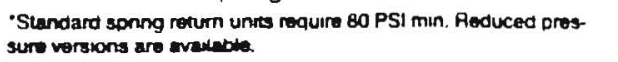 \\
\hline Media & Air or non-corrosive gas. \\
\hline \multirow{4}{*}{$\begin{array}{l}\text { Temperature } \\
\text { Aange }\end{array}$} & $0^{\circ}$ To $212^{\circ} \mathrm{F}\left(-18^{\circ}\right.$ to $\left.100^{\circ} \mathrm{C}\right)$ Actuator Only \\
\hline & To $130^{\circ} \mathrm{F}\left(54^{\circ} \mathrm{C}\right)$ Actuator with G.P. or X.P. Solenoid \\
\hline & $\begin{array}{l}\text { To } 160^{\circ} \mathrm{F}\left(71^{\circ} \mathrm{C}\right) \text { Actuator With W.P. or } \\
\text { NEMA IV/VII Solenoid }\end{array}$ \\
\hline & $\begin{array}{l}\text { High temperature option to } 250^{\circ} \mathrm{F} \text { continuous. to } \\
300^{\circ} \mathrm{F} \text { intermittent (without solenoid) } \\
\text { Low temperature option to }-70^{\circ} \mathrm{F} \text { (without solenoid) }\end{array}$ \\
\hline Rotation & $\begin{array}{l}\text { Actuators rotate in counter-ctockwise direction } \\
\text { when the outer air connection is pressurized. }\end{array}$ \\
\hline \multicolumn{2}{|l|}{ Movement } \\
\hline Sizes 10-35: & $\begin{array}{l}90^{\circ} \text { with up to } 1^{\circ} \text { overtravel counterclockwise } \\
\text { (valve open) and up } 103^{\circ} \text { oventravel clockwise } \\
\text { (valve closed) }\end{array}$ \\
\hline Sizes 40-50: & $90^{\circ}$ with up to $1 \frac{1}{2}{ }^{\circ}$ overrun each end \\
\hline SUPPLY AIR- & $\begin{array}{l}\text { - The Series } 39 \text { Actuator is factory lubricated. For } \\
\text { optimum pertormance. standard filtered and lubri- } \\
\text { cated air is recommended. }\end{array}$ \\
\hline
\end{tabular}




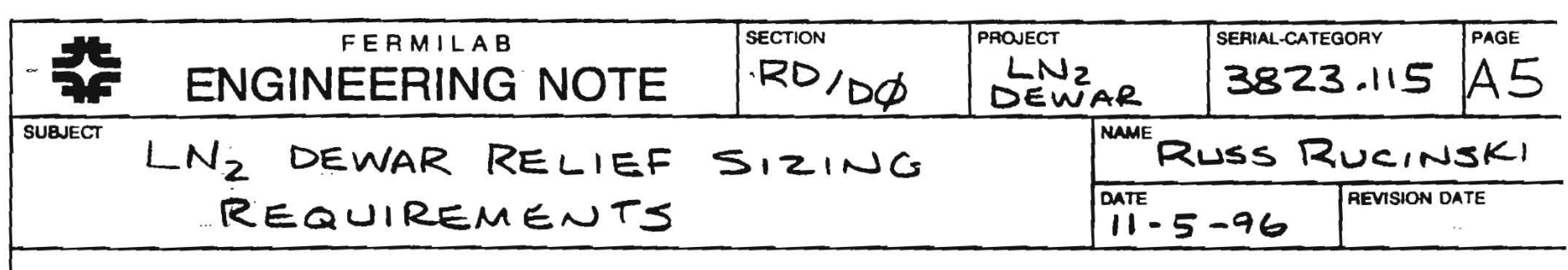

CASE 5: Filling a warm dewar

IH HE INITIAL FILL OR ANY WARM FILL of IH IS DEWAR" WILL 'BE SUPERVISED dY. EERMILAB PERSONNEL (MYSELF OR A DESIGNER).

Regardless of our presence however, THE VESSEL IS PROTECTED BY THE Automatic FILL SHUT-OFF VALVE THAT WAS DESCRIBED IN CASE 4. 


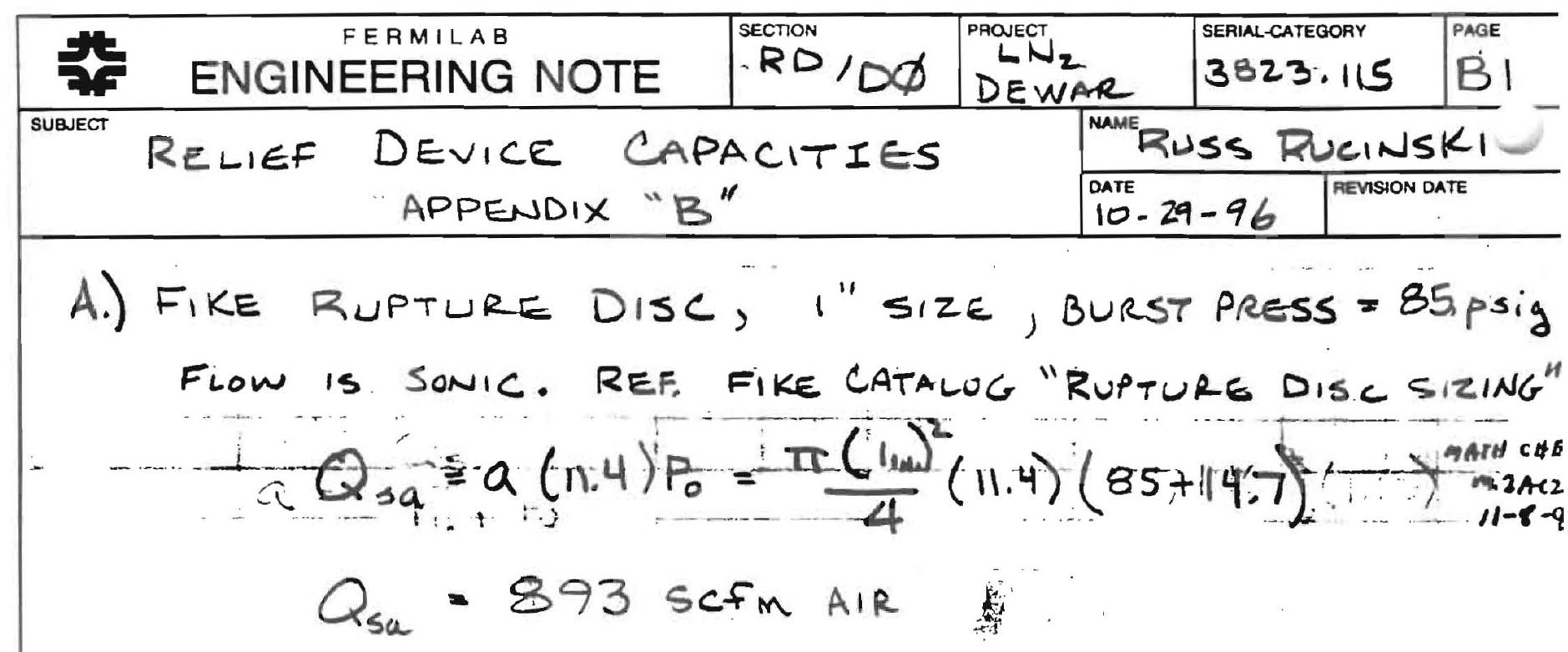

B.) AGCO $81 B 88-9$, S.P. $=60$ psig from Aglo catalog SERIES so reLIEFS;

$$
A=\frac{V \sqrt{M T Z}}{6.32 C K P_{1}}
$$

Re-Arranging the terms, $V=\frac{6.32 \mathrm{CKP}, A}{\sqrt{M T Z}}$

$$
V=\frac{6.32(356)(.816)(78 \mathrm{Psig}+14.7)\left(.196 \mathrm{in}^{2}\right)}{\sqrt{(29)\left(520^{\circ} \mathrm{R}\right)(1.0)}}
$$

$V=271.6 \mathrm{sCFM}$ AIR E MAWP OF VESSER. 


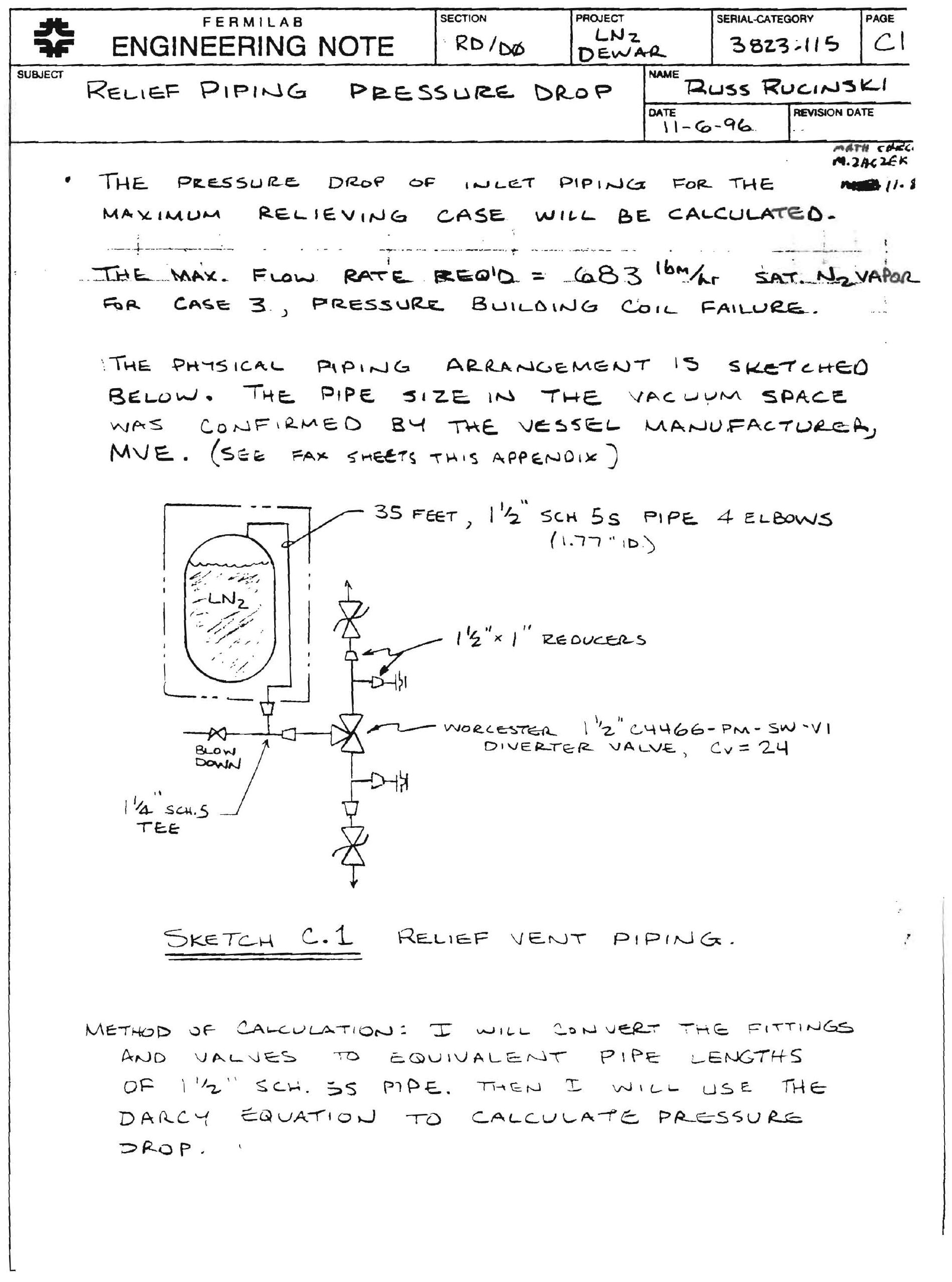




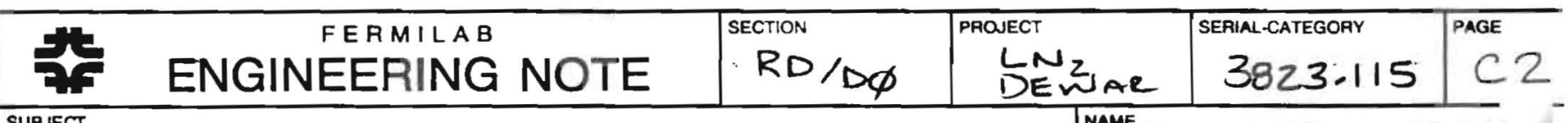

$$
\begin{aligned}
& \text { DATE } 11-6-96 \text { REVISION DATE } \\
& L=\frac{\sum K D}{F}=\frac{48 F_{T} D}{F_{T}}=48 D \\
& \text { EAm. } 2-4 \\
& L_{\text {ELBow }}=48\left(\frac{1,77}{12} \mathrm{ct}_{t}\right)=7.1 \text { FEET } 11 / 2 \text { "sCH. } 5 \mathrm{~s} \text { PIPE } \\
& K_{T E E}=60 F_{T} \quad K_{1 / 2}=K_{1 / 4}\left(\frac{d_{1 / 2}}{d_{1 / 4}}\right)^{4} \quad \text { LETT }\left.F_{T}\right|_{1 / 4}=\left.F_{\tau}\right|_{1 / 2}
\end{aligned}
$$

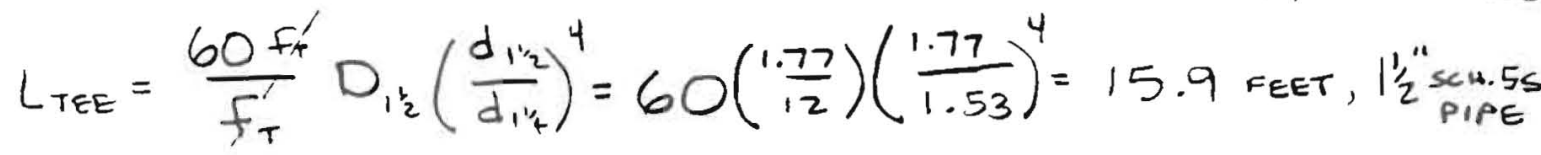

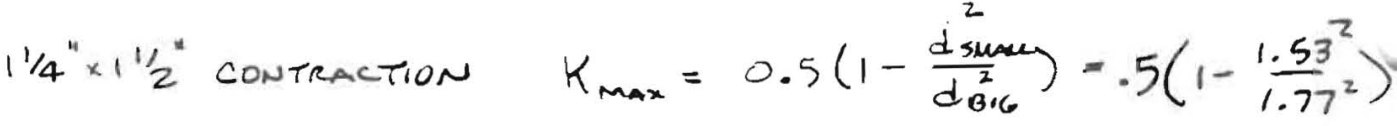

$$
\begin{aligned}
& K_{\text {max }}=.1264 \text { in Terms of 1"4" ait } \\
& L_{\text {RED }}=.1264\left(\frac{1.77}{12}\right)\left(\frac{1.77}{1.53}\right)^{4}=0.03 \mathrm{fte} \text {. NEGUGGBLE. } \\
& K_{\text {max }}=.5\left(1-\frac{1.09^{2}}{1.77^{2}}\right)=.31038 \text { in means or I" pipe } \\
& L_{\text {gEO }}=.31038\left(\frac{1.77}{12}\right)\left(\frac{1.77}{1.09}\right)^{4}=.318 \mathrm{Ft} \text {. }
\end{aligned}
$$

diverter valve: $C_{V}=24$ from worcester catalog.

$$
\begin{aligned}
& C_{V}=\frac{29.9 d^{2}}{\sqrt{k}} \text { EON 2-6 crane. } \\
& K_{\text {awe }}=\left[\frac{29.9 d^{2}}{C_{1}}\right]^{2}=\left[\frac{29.9(1.77)^{2}}{24}\right]^{2}=15.23 \\
& L_{\text {AWE }}=\frac{k \Delta}{F}=\frac{(15.23)\left(1 \frac{77}{12}\right)}{.021}=107 \text { sET }
\end{aligned}
$$

TEE P OUTLET OF DIVERTER:

$$
\begin{aligned}
& K_{T_{\text {Hind }}}=20 \mathrm{Ft}_{\mathrm{t}} \\
& L=20\left(\frac{1.77}{12} \mathrm{Ft}\right)=3.0
\end{aligned}
$$




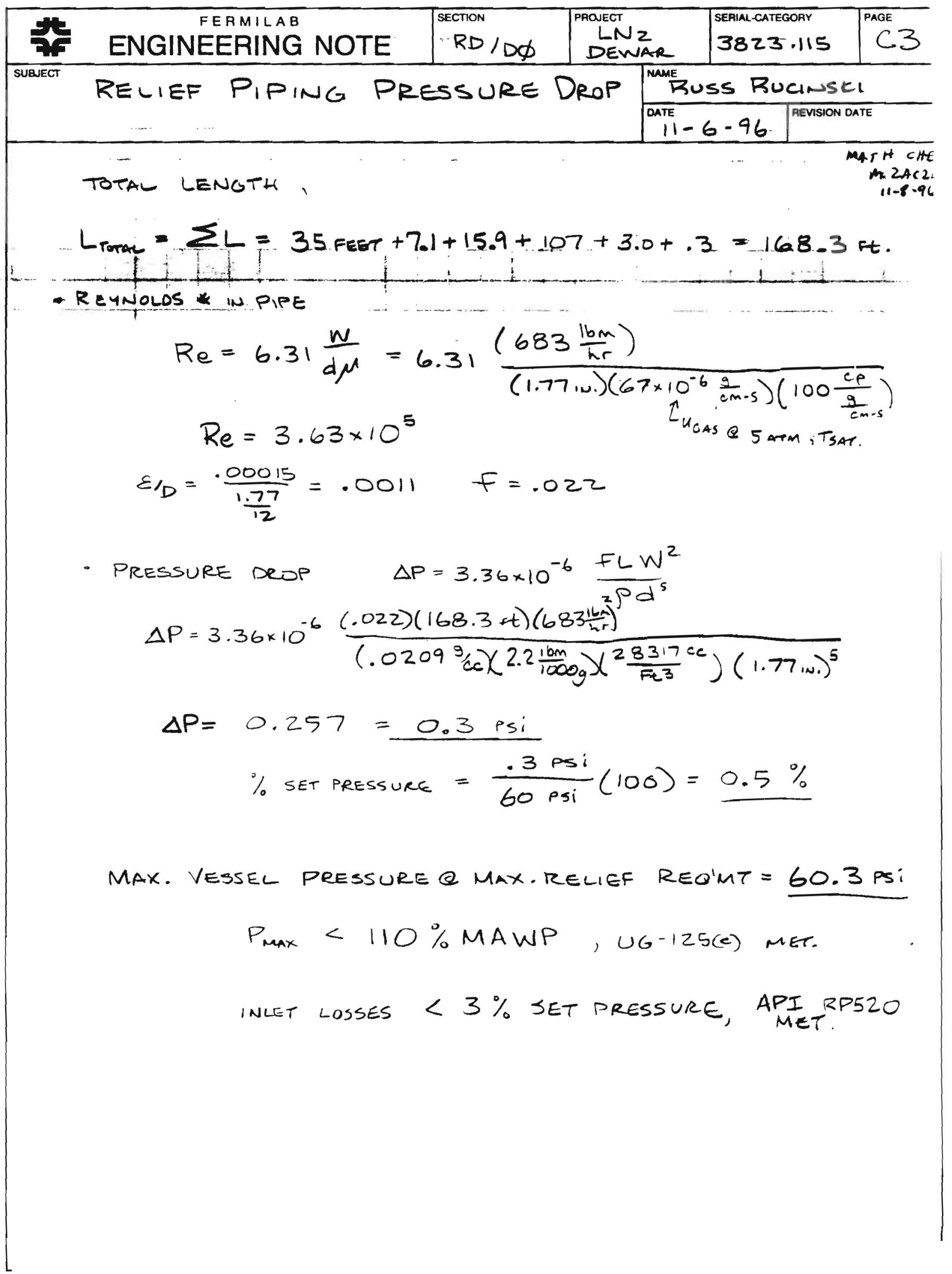


D-Zero Department FAX Cover Page Fax Number (630) 840-8481

To: PEGGY ERPELAK, MVE From: RUSS RUCINSKI [Prowl 630-840-2898] Subject: VLS -9000 , SNalO9 RELLIEF PIPE.

PegGy,

What size is the relief Valve inlet PIPING For This Tank? Please Fax

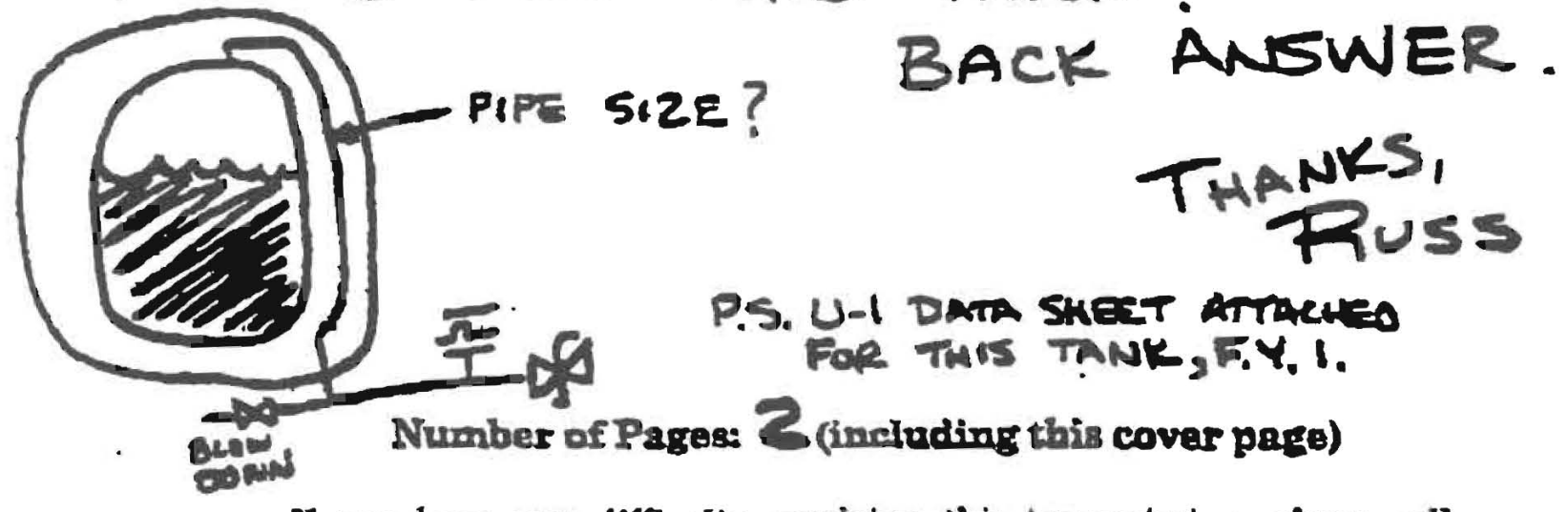

If you have any difficulty receiving this transmission, please call Barbara Edmonton of (630) 840-3695. 
CS

MVE, Inc.

107 Seventh Street NW

P.O. Box 234

New Prague, MN 56071-0234

ph /612.758.4484 $\cdot$ fx/612.758.8293

FAX COVER SHEET

DATE: $10-30-96$

To: Russ RuTAN SK 1 fax: $630-840-8481$

FERmi LAB

FROM: GARY PAULY PHONE: 612-758-8277

ME INC.

FAX: $\quad$ 612-758-8215

RE:

CC:

Number of pages including cover sheet

Message:

Russ

The internal Vent Pipe for the VLS-9000 SIN 109 IS 11/2" P.S. (1.90"0D)

ScH. 5

G.Pauny 
Prepared By Russ RucinskI

Reviewed By cryeifhr

Div/Sec Head tetiente
Date $11-7-96 \mathrm{Div} / \mathrm{Sec}$ RD/D.ZERO MECH. Date $\frac{1 / 17 / 97}{1 / 24 / 97}$ Div/Sec BD/Cryogonics

\section{Identification and Verification of Compliance}

Fill in the Fermilab Engineering Conformance Label information below:

This vessel conforms to Fermilab ES\&H Manual Chapter 5033

Vessel Title LN $L$ STORAGE DEWAR

Vessel Number RDV 2045

Vessel Drawing Number NA

Internal MAWP $>$ RELIEF SETTING

External MAWP $N / A$

Working Temperature Range -320 oF $100 \quad$ OF

Designer/Manufacturer MVE CRYOGENICS

Date of Manufacture_ 1985 Acceptance Date

Director's signature (or designee) if vessel is for manned area and requires an exception to the provisions of this chapter.

$\begin{array}{lll}\text { Amendment No.: } & \text { Reviewed By: }\end{array}$

Laboratory location code RPIS - 325 (DAB)

Laboratory property number NAL 51464

Purpose of vessel INSULATING VACUUM 
DRG. 3823.115-ME-3172235HT.1, FLOW SCHEMATIC. DQ ASS'Y BLDG. FILES. ALSO KEPT ELECTRONICALG IN XDCS, DQ MECH. VAULT

2. Design Verification

Provide design calculations in the Nore Appendix. Commercially ManUfactured DEwar
BUILT To INDUSTRY STANDARDS.

3. System Venting Verification SEE ATTACHED VENDOR INFO. Can this vessel be pressurized either internally or extemally? [ ] Yes
If yes, to what pressure?

List all reliefs and settings. Provide a schematic of the relief system components and appropriate calculations or test results to prove that the vessel will not be subjected to pressures greater than $110 \%$ beyond the maximum allowable internal or extemal pressure.

4. Operating Procedure Section

Relief Pressure Setting Flow Rate Size

Manufacturer

6"LIFT PLATE_I PSI N/A FLOW AREA $=7.625 \mathrm{in}$

Is an operating procedure necessary for the safe operation of this vessel?

Yes No (If "Yes", it must be appended)

Is a testing procedure necessary for the safe acceptance testing (proof testing) of this vessel'? [] Yes $\mathbb{X}$ No

If yes, the written procedure must be approved by the Division Head prior to testing and supplied with this Engineering Note. 
5. Weijing Tnionmat:

Has the vessel been fabricated in a Fermilab shop? [ ] Yes Wo

If "Yes," append a copy of the welding shop statement of welder qualification.

6. Exceptional Existing Used and Non-Manned Area Vessels

Is this vessel or any part thereof in the above categories?

Yes No

If "yes" follow the Engineering Note requirements for documentation and append to note.

THIS VESSEL IS AN EXISTING LN2 DEWAR

THAT WAS MOVED FROM THE E-706

EXPERIMENT (NOW DE-COMMISSIONEO) TO

THE D-ZERO EXPERIMENT.

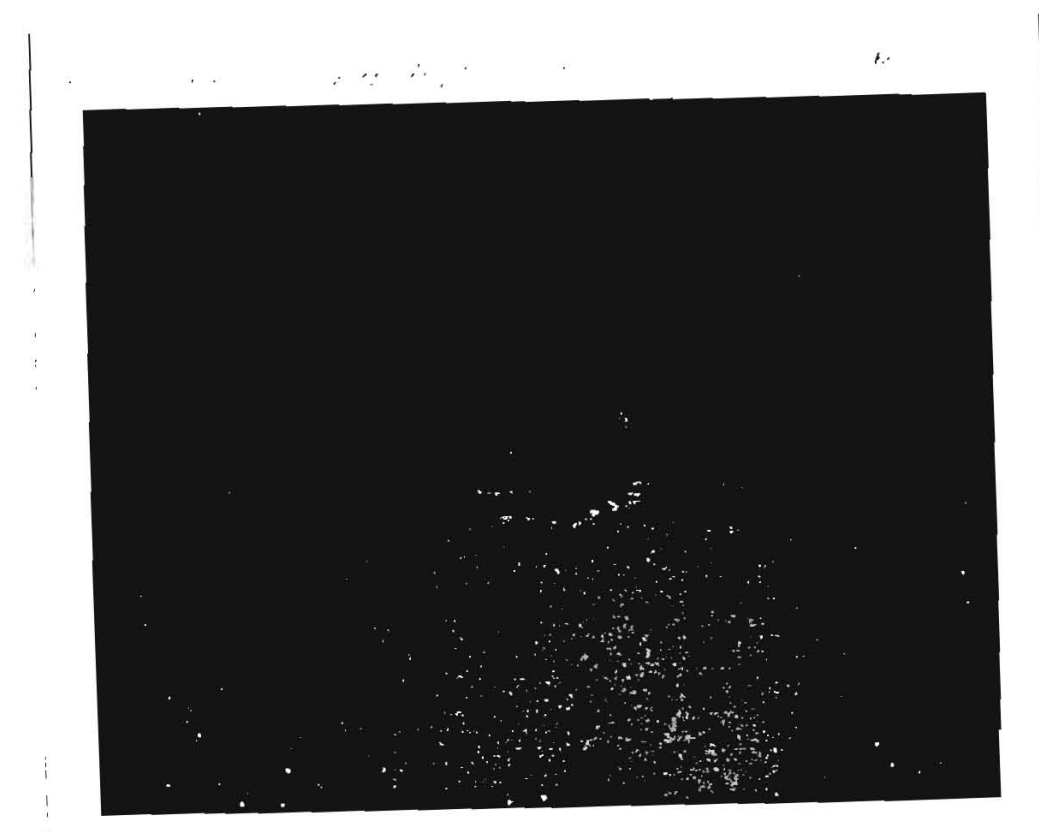

THIS PHOTO VERFIE THE LIFT PLATE ON THE VACUUM VESSEL IS NOT BOLTED DOWN. it measures $8 \%$ OUTSIDE DIA. BY ABOUT $3 / 8^{\prime \prime}$ THICK PHOTO TAKEN $11-8-96$ (AT TOP OF DEWAR) R.RuCinsk 
From ORIGITAR 14.1 PRESSURE

VESSEL ENGINEERING NOTE

OUTER VESSEL REIIEF

FOR RDZ045 BY D. BURKE $14 \mid 41$

Required area:

$$
\begin{array}{ll}
\text { A } & =2.4 \mathrm{E}-4 \mathrm{in}^{2} / \mathrm{lb}-\mathrm{H}_{2} \mathrm{Ocap} \times \text { Density } \mathrm{H}_{2} \mathrm{O} \times \text { Volume } \\
\text { Density } \mathrm{H}_{2} \mathrm{O}= & 8.36 \mathrm{lb} / \mathrm{gal} \text { of } \mathrm{H}_{2} \mathrm{O} \\
\text { Volume } & =9000 \mathrm{gal} \\
\text { A } \quad & 2.4 \mathrm{E}-4(8.36)(9000)=18.1 \mathrm{in}^{2} \\
& A=18.1 \mathrm{in}^{2} \text { required }
\end{array}
$$

Actual area: $A_{t}$

$\mathrm{Dia}=7.625 \mathrm{in}$, flow area of the relief device

$A_{t}=45.66 \mathrm{in}^{2}$, area of the relief device

$$
\underline{A}_{t}>A \quad 45.66>18.1
$$

The outer vessel relief device is a lift plate tested by the manufacturer to lift at 1 psig. 


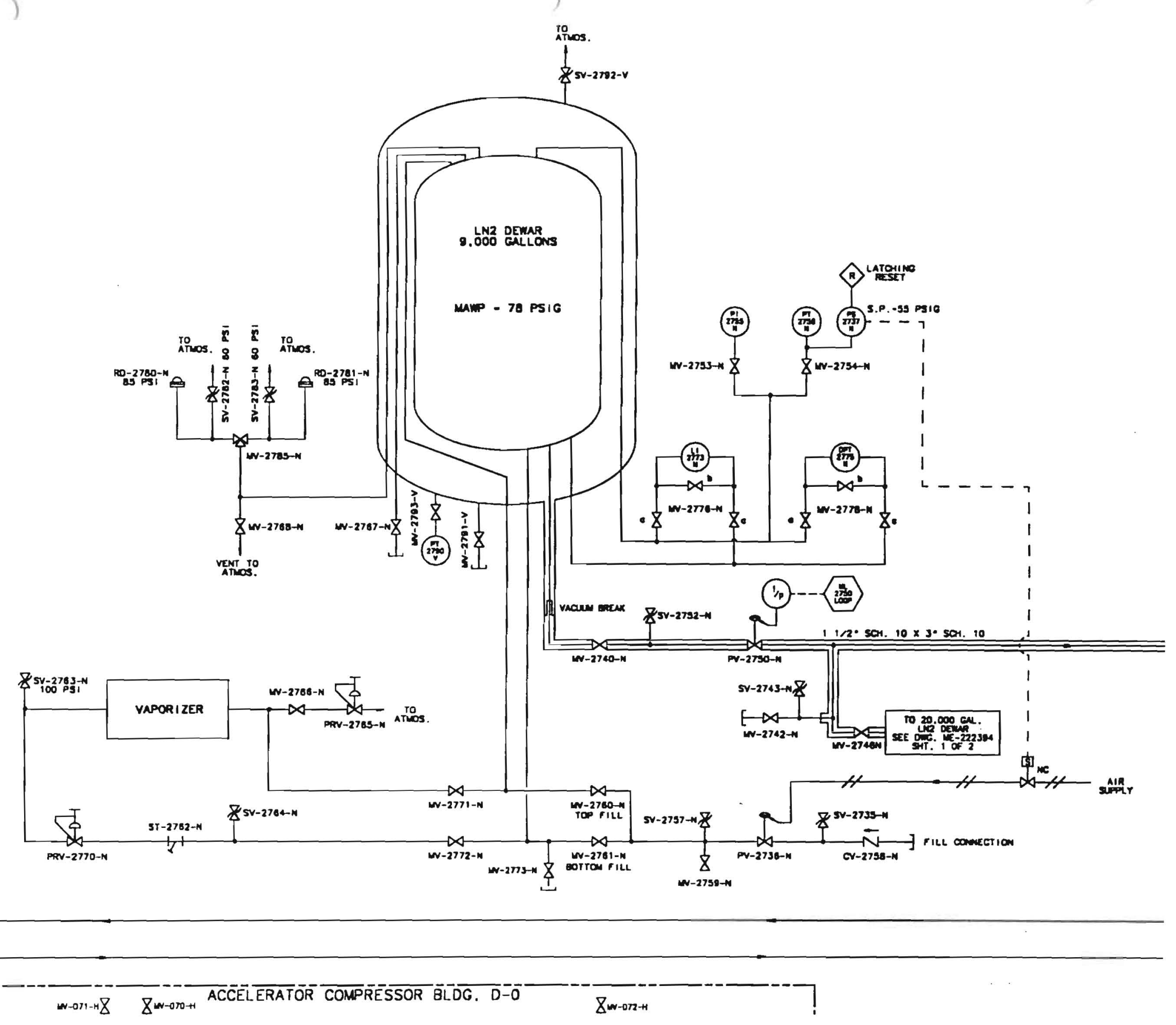


MVE, Inc.

TECHNICAL MANUAL

VERTICAL STORAGE SYSTEMS

VCSNLS

SERIES

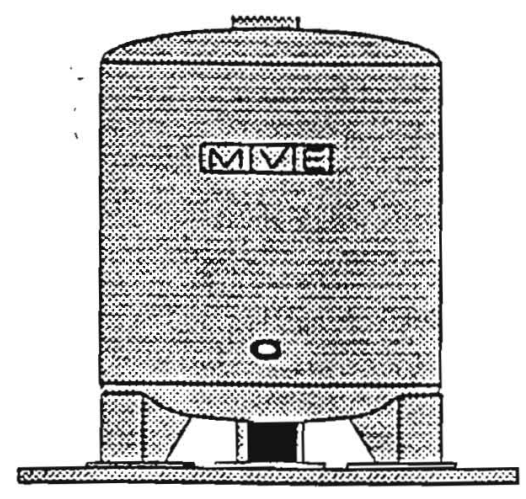

MANUAL \# AAA0429 
MVE customer stations are safely designed with the following features: $\quad \cdots$

1. A vacuum maintenance system specifically designed to provide long life and all possible safety provisions.

2. Safety relief devices to protect the pressure vessel and vacuum casing, sized and selected in accordance with ASME standards to include a pressure control valve and rupture disc to protect the pressure vessel and a reverse buckling rupture disc or llft plate to protect the vacuum casing from overpressure. While MVE equipment Is designed and built to the most rigid standards, no piece of mechanical equipment can ever be made $100 \%$ foolproof. Strict compliance with proper safety and handling practices is necessary when using a Vertical Customer Statlon (VCS) or Vertical Llquld Statlon (VLS). We recommend that all our customers emphasize safety and safe handling practices to all thelr employees and customers. While every possible safety feature has been designed into the unit and safe operatlons are anticipated, it is essentlal that the user carefully read and fully understand all WARNING and CAUTION notes listed in this safety summary and enumerated below. Also read to fully understand the information provided In the Safety Bulletins for Oxygen and Inert Gases located in Appendlx C of this Manual. Periodic review of the Safety Summary is recommended.
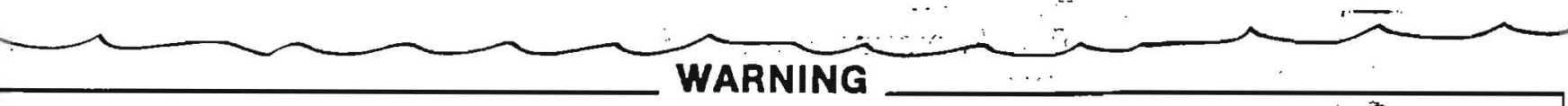

$\therefore-$

$\because \ldots$

$\therefore$

Excess accumulation of oxygen creates an oxygen enfiched atmosphere (defined by the Compressed Gas Association as an oxygen concentration above $23 \%$ ). In an oxygen enrlched atmosphere, flammable items burn vigorously and could explode. Certain items considered non-combustible in alr may burn rapidly in such an environment. Keep all organic materials and other flammable substances away from possible contact with oxygen; particulariy oil, grease, kerosene, cloth, wood, paint, tar, coal dust, and dirt which may contaln oil or grease. DO NOT permit smoking or open flame in any area where oxygen is stored, handled, or used. Failure to comply with this warning may result in serious personal injury.

WARNING

Nitrogen and argon vapors in alr may dilute the concentration of oxygen necessary to support or sustain life. Exposure to such an oxygen deficient atmosphere can lead to unconsclousness and serious injury, including death.

\section{WARNING}

Before removing parts or loosening fittings, empty the container contents of llquid and release the vapor pressure in a safe manner. External valves and fittlngs can become extremely coid and may cause painful burns to personnel unless properly protected. Personnel must wear protective gloves and eye protection whenever removing parts or loosening fittings. Failure to do so may result in personal injury because of the extreme coid and pressure in the vessel.

\section{WARNING}

Accidental contact of liquid gases with skin or eyes may cause a freezing injury similar to a burn. Handle liquid so that it will not splash or spill. Protect your eyes and cover skin where the possibilIty of contact with llquid, cold pipes and cold equipment, or cold gas exists. Safety goggles or a face shield should be worn if liquid ejectlon or splashing may occur or cold gas may issue forcefuliy from equipment. Clean, insulated gioves that can easily be removed and long sleeves are recommended for arm protection. Cuffless trousers should be worn outside boots or over the shoes to shed spilled liquid. 


\section{1-1. GENERAL}

The Minnesota Valley Engineering (MVE) cryogenic container is a compact, self-contained, and statlonary liquid gas storage and delivery system. They are multi-layer or perite insulated, medium pressure containers designed for storage of liquid argon, nitrogen, or oxygen, and for product withdrawal in either gaseous or liquid form.

The model designation VLS indicates that the cryogenic container is a "Vertical Luquid Station" intended for liquid withdrawal only. There are 6 standard models currently available, in multi-layer insulation only.

The model designation VCS indicates that the cryogenic container is a "Vertical Customer Sta. tion" intended for either gaseous or llquid withdrawal. The model numbers, l.e., $300,500,900$, etc., indicate the approximate net capacity (In gallons) of the particular.vCS.

\section{1-2. PHYSICAL DESCRIPTION}

An MVE Station is designed for long-term storage of cryogenic Ilquefled gases under pressure in the range of 5 to 250 PSIG. Operation of the station is fully automatic with the unit's regulator systems set to maintain preset pressure and flow conditions into a customer's pipeline. While hardware may vary slightly from model to model, each unit essen. tially performs the same functions.

The VCS or VLS is comprised of an alloy steel inner tank encased in an outer carbon steel vacuum shell. The insulation system between the inner and outer containers consists of multiple layer or powder insulation and high vacuum to ensure long holding time. The insulation system, designed for long- term vacuum retention, is permanently sealed at the factory to ensure vacuum integrity. The units have a tank pressure relief device which is set at the factory. As a secondary pressure relief device, the container is further protected from overpressurization by a rupture disc. The bursting disc will rupture completely to relieve inner tank pressure in the event the tank relief valve fails and pressure exceeds the rupture disc setting. The vacuum space is protected from overpressurization by use of a tank annulus rupture disc assembly. Pressure relief devices used on VCS' or VLS' meet the requirements of CGA Pamphlet S1.3, "Pressure Relief Device Standards, Part 1, for Stationary Vessels."
The VCS or VLS is leg mounted. On the smaller units (300-1500 gallon), llfting lugs are secured to one leg and to the top head of the contalner. On units larger than 1500 gallon, the llfting lugs are located on the top and bottom heads. The lifting lugs are provided to facilltate handling. Moving requires the use of a crane and adherence to specific rigging instructlons (see Chapter 4 In this manual for detalls on handling).

Controls used to operate the system are mounted under the customer statlon. The pressure gauge and liquid level gauge are located at eye level on the contalner for ease of vlewing.

The VCS has a dual regulator system which uses both an economizer regulator and a pressure building regulator. The economizer regulator automatically allows vapor space gas to be introduced preferentlally into the final line or gas use circuit when the customer station pressure exceeds the regulator setting. The pressure building regulator is used to maintain a minimum set pressure in the vessel. An optional third regulator, the final line regulator, can be used to control pressure and gas flow into the customer's line.

The VLS units only have a pressure building system, since they are designed for liquid use exclusively.

The vessels also contain a pressure building coil and a strainer assembly. The pressure bullding coil vaporizes the liquid to bulid up pressure in the tank. The strainer assembly protects the pressure building system from damage by contaminants.

Some VCS contalners are shipped with an ambient air vaporizer. Containers are ready for service as soon as they are erected, bolted to the foundation, and have the process pipeline connected to the vaporizer outlet. VCS Models 315 through 1500 are shipped with the vaporizer mounted on the side of the tank (unless a tank is ordered without the vaporizer). Due to dimensional limitatlons for shipping, vaporizers are not factory installed on VCS's larger than Model 1500. On VCS's larger than Model 1500 , the vaporizer is located adjacent to the container on the foundation.

The normal operating pressure range of a VCS is from approximately 50 to approximately $150 \mathrm{PSI}$, with a maximum working pressure of approximately $250 \mathrm{PSI}$. Operating pressure can be increased or decreased as desired by adjusting the regulators. Vessels with a higher pressure rating are available.

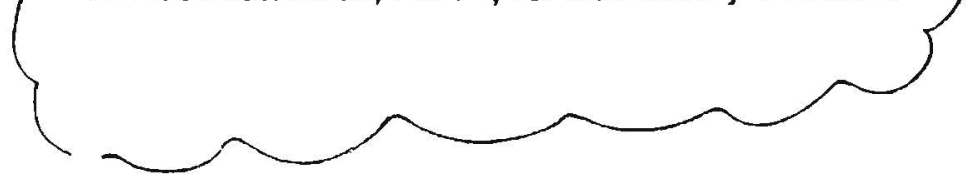


Figure 6-7. Parts Identification Vlew

VCS $9000-S S \cdot 250-M V E-0$

VLS 9000-SS-75-MVE-0

VCS 9000-SP-250-MVE-0

VCS $9000-N P-250-M V E-0$

VLS 9000-SP-75-MVE-0
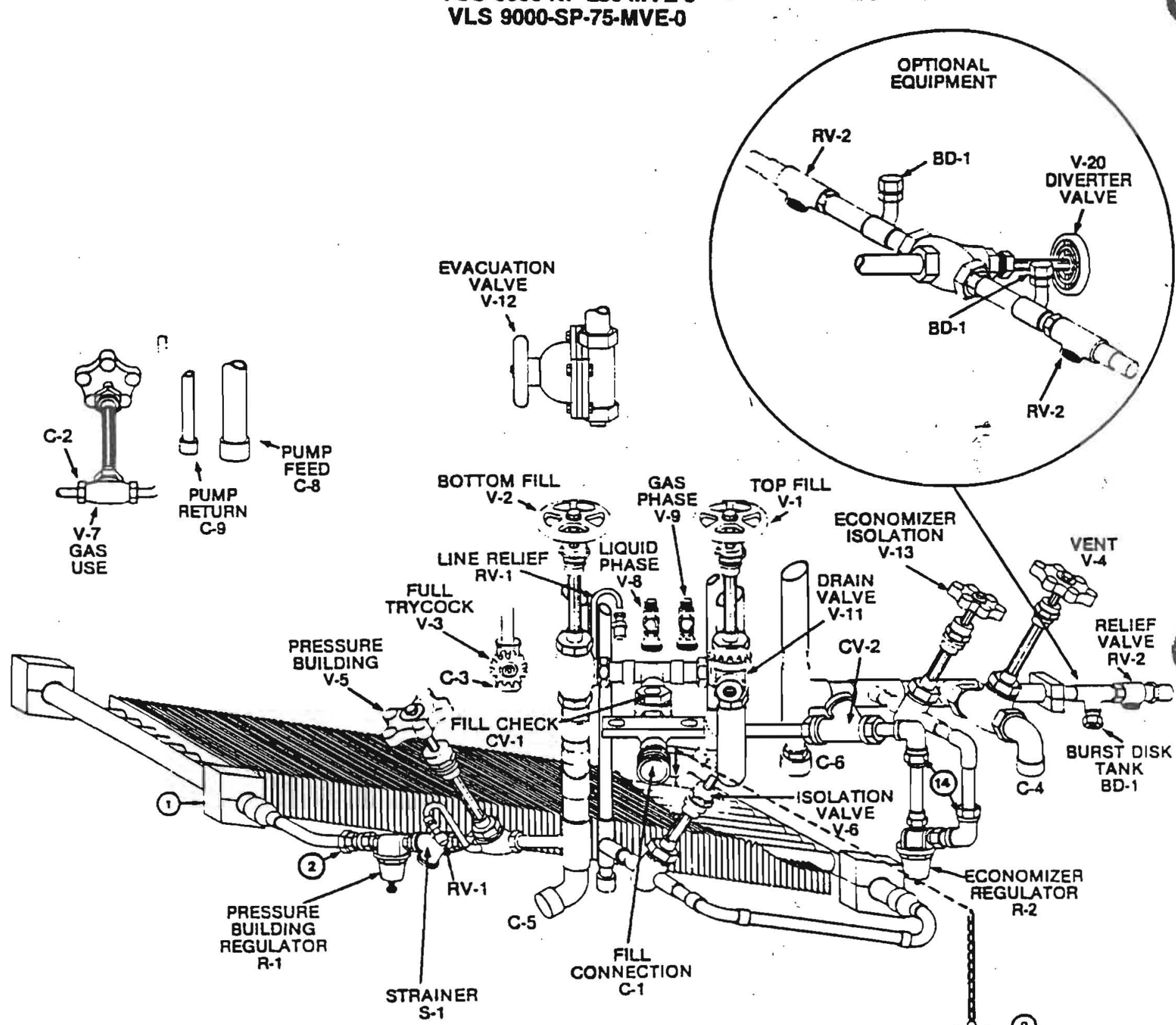

$y 3$

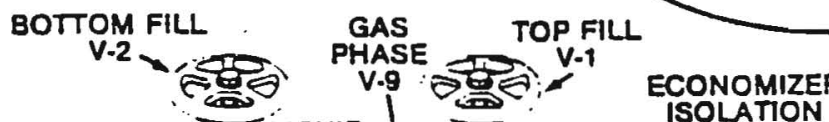

LINE RELIEF LUAUID
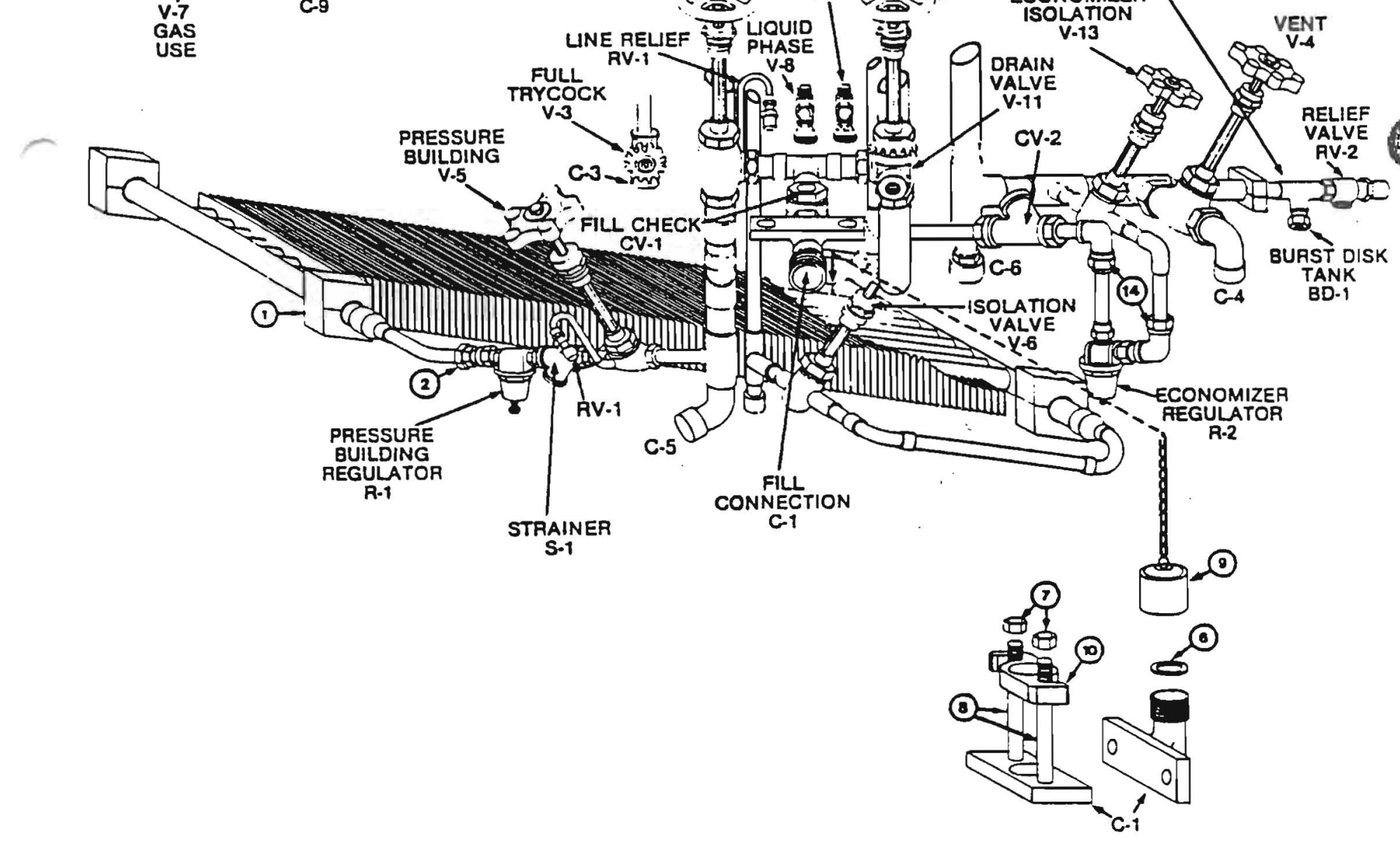

NOTE: VLS models will not have an economizer or gas use circuit. 
Tabie 6-13. Parts Identlfication List 9000 Gailon Units

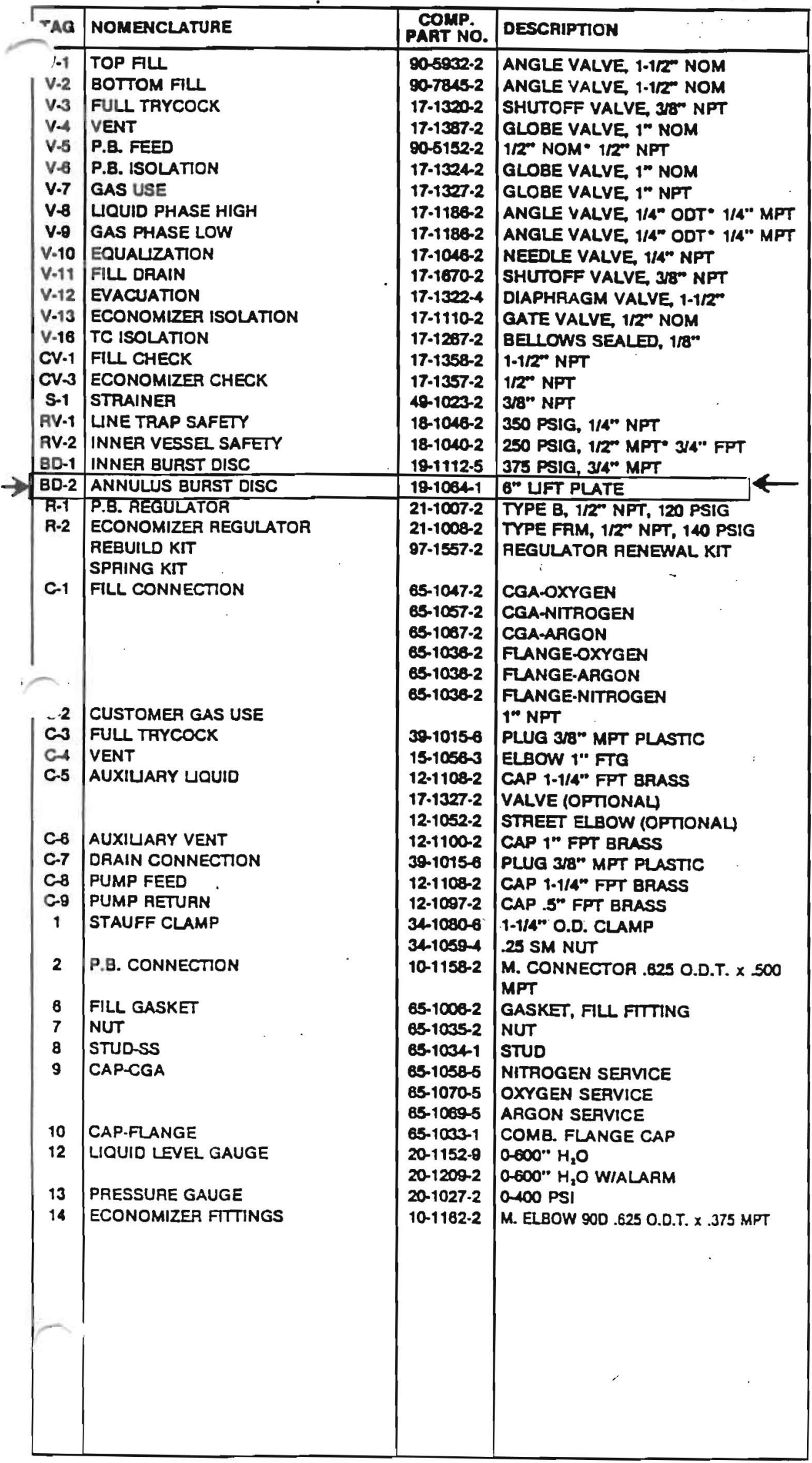

Table 6-14. Llquid Contents Chart VCS $9000-5 S-250-M V E-0$ VLS 9000-SS-75-MVE-0 VCS 9000-SP-250-MVE-0 VCS 9000 -NP-250-MVE-0 VLS 9000-SP-75-MVE-0

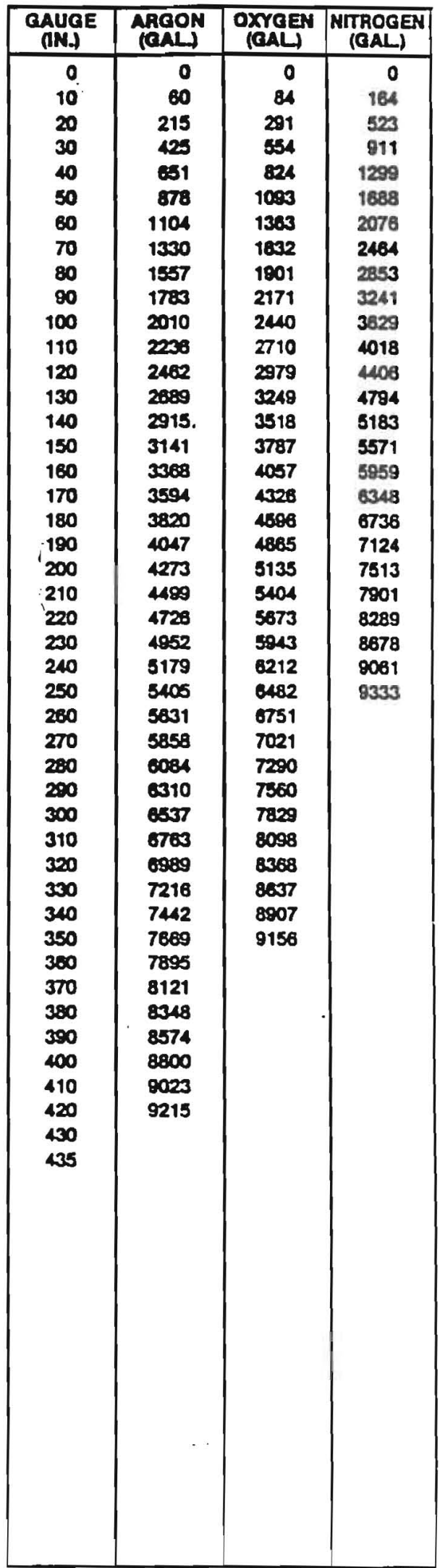

NOTE: Contents based on llquid saturated at O PSIG. 